ANL-HEP-TR-01-113

\title{
HIGH ENERGY PHYSICS DIVISION SEMIANNUAL REPORT OF RESEARCH ACTIVITIES
}

January 1, 2001 - June 30, 2001

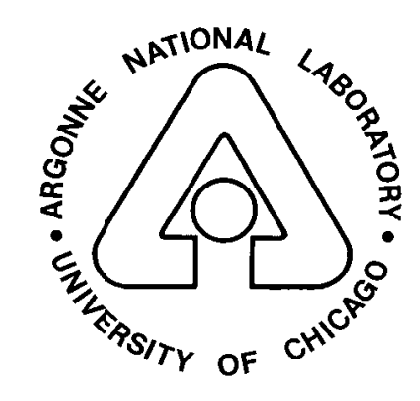

ARGONNE NATIONAL LABORATORY

Argonne, Illinois

Operated by THE UNIVERSITY OF CHICAGO for the

U.S. DEPARTMENT OF ENERGY

under Contract W-31-109-Eng-38 
Argonne National Laboratory, with facilities in the states of Illinois and Idaho, is owned by the United States government, and operated by The University of Chicago under the provisions of a contract with the Department of Energy.

DISCLAIMER
This report was prepared as an account of work sponsored by an agency of
the United States Government. Neither the United States Government nor
any agency thereof, nor The University of Chicago, nor any of their
employees or officers, makes any warranty, express or implied, or assumes
any legal liability or responsibility for the accuracy, completeness, or
usefulness of any information, apparatus, product, or process disclosed, or
represents that its use would not infringe privately owned rights. Reference
herein to any specific commercial product, process, or service by trade name,
trademark, manufacturer, or otherwise, does not necessarily constitute or
imply its endorsement, recommendation, or favoring by the United States
Government or any agency thereof. The views and opinions of document
authors expressed herein do not necessarily state or reflect those of the
United States Government or any agency thereof, Argonne National
Laboratory, or The University of Chicago.

Available electronically at http://www.doe.gov/bridge

Available for a processing fee to U.S. Department of Energy and its contractors, in paper, from:

U.S. Department of Energy

Office of Scientific and Technical Information

P.O. Box 62

Oak Ridge, TN 37831-0062

phone: (865) 576-8401

fax: (865) 576-5728

email: reports@,adonis.osti.gov 
Argonne National Laboratory

9700 South Cass Avenue

Argonne, Illinois 60439

\title{
HIGH ENERGY PHYSICS DIVISION SEMIANNUAL REPORT OF RESEARCH ACTIVITIES
}

January 1, 2001 - June 30, 2001

Prepared from information gathered and edited by The Committee for Publications and Information:

\author{
Members: $\quad$ P. Malhotra \\ J. Norem \\ R. Rezmer \\ R. Wagner
}

December 2001 


\begin{abstract}
This report describes the research conducted in the High Energy Physics Division of Argonne National Laboratory during the period of January 1, 2001 through June 31, 2001. Topics covered here include experimental and theoretical particle physics, advanced accelerator physics, detector development, and experimental facilities research. Lists of Division publications and colloquia are included.
\end{abstract}





\section{Table of Contents}

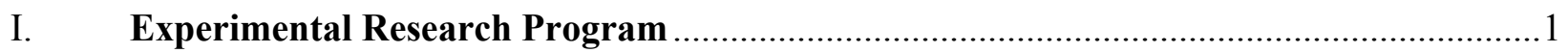

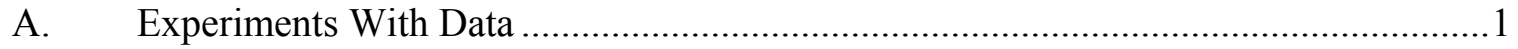

1. Medium Energy Polarization Program …………………...............................

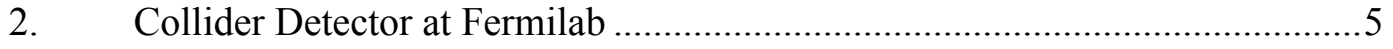

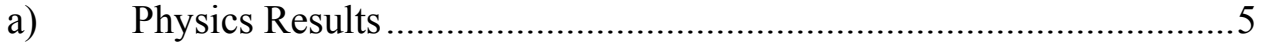

b) The CDF Run 2 Commissioning .......................................................

3. The CDF Upgrade Project ......................................................................

a) Run II Planning ............................................................................

4. Non-Accelerator Physics at Soudan ............................................................

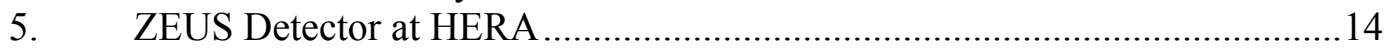

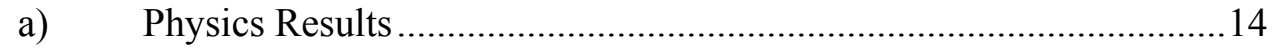

b) HERA and ZEUS Operations........................................................19

B. Experiments In Planning Or Construction …………….......................................19

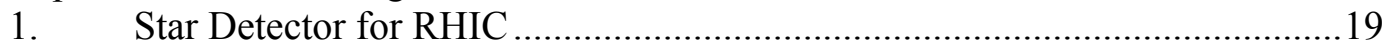

2. MINOS Main Injector Neutrino Oscillation Search ........................................22

3. ATLAS Detector Research \& Development..................................................26

a) Overview of ANL ATLAS Tile Calorimeter Activities......................26

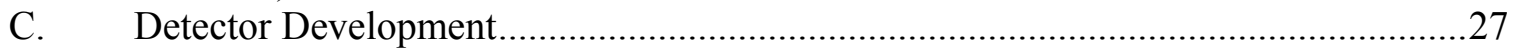

1. ATLAS Calorimeter Design and Construction...............................................27

a) Submodule Construction .................................................................2

b) Module Assembly and Shipping ………………………………......28

c) Module Shipping ...............................................................................29

d) Instrumentation and Testing ...............................................................30

e) Test Beam Program ............................................................................31

f) Engineering and Design and Analysis................................................33

2. Computational Projects..............................................................................34

a) ATLAS Computing ………………………………………………....34

3. MINOS Detector Development …………………......................................36

4. Electronics Support Group............................................................................37

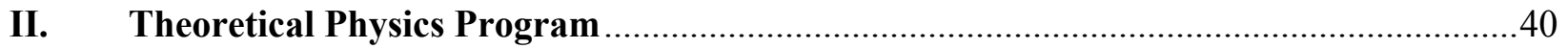

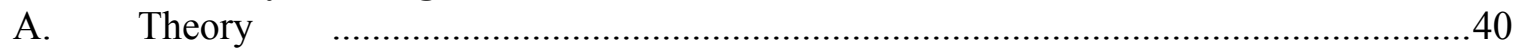

1. Upsilon Decay to a Pair of Bottom Squarks ………………………………....40

2. Massive Lepton-Pair Production at Large Transverse Momentum .....................42

3. Argonne 2001 Theory Institute on Supersymmetry and Higgs Physics .............43

4. Compatibility of Approaches to Heavy-Quark Fragmentation............................43

5. Resummation of Large Corrections to Heavy-Quarkonium Decays ...................43

6. Computational Physics.................................................................................4

7. Brane Effects on Large Extra Dimension Scenarios............................................45

8. The Chiral Anomaly and the Regge Limit of QCD ……………………….......48

9. Negative Probability and Uncertainty Relations.................................................49 


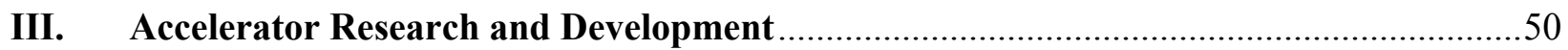

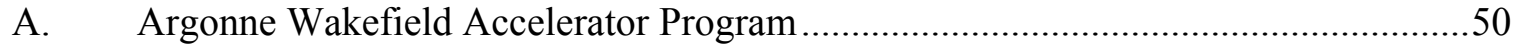

1. The Argonne Wakefield Accelerator Status. ..................................................50

2. The AWA Experiments ............................................................................51

3. New Accelerator Concept Developed........................................................51

4. Design of precision Wakefield Measurement System for Engineering Test

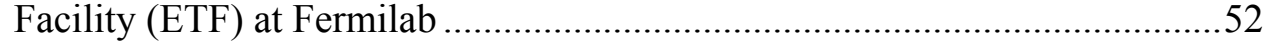

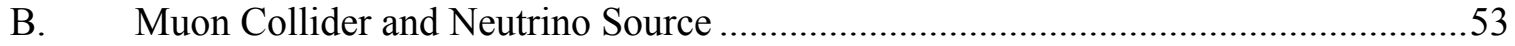

a) Instrumentation for the Neutrino Source......................................53

b) An $\mathrm{e}^{+} \mathrm{e}^{-}$Collider in the VLHC Tunnel...........................................54

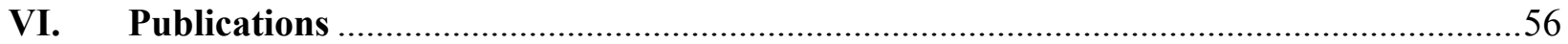

A. Books, Journals, and Conference Proceedings..........................................................56

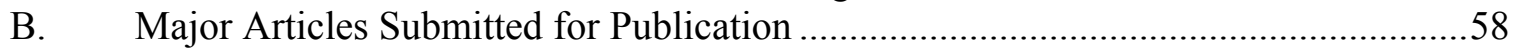

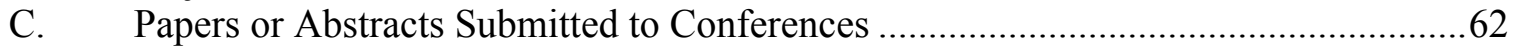

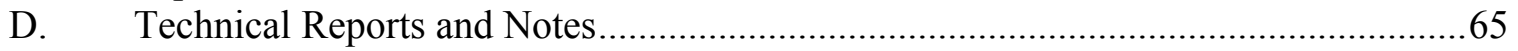

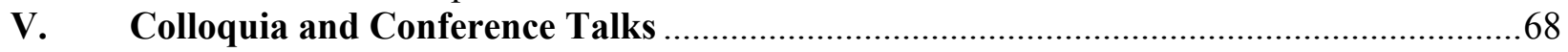

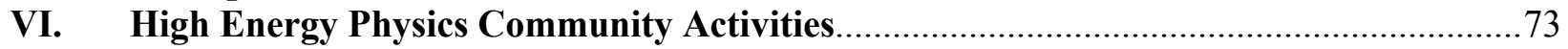

VII. High Energy Physics Division Research Personnel ......................................................76 


\section{EXPERIMENTAL RESEARCH PROGRAM}

\section{I.A. EXPERIMENTS WITH DATA}

\section{I.A.1 Medium Energy Physics Program}

During the period Jan. - June 2001, ANL physicists completed analysis and a technical note about data from the AGS polarimeter, and nearly finished a major paper describing a spin experiment at the AGS. This latter experiment suggests a possible polarimetry technique for high energy proton accelerators or colliders, such as RHIC and HERA. Papers from past experiments with the Crystal Ball and from recent measurements with STAR were also submitted and published.

The major physics goal for the group is to better understand the gluon and quark contributions to the nucleon spin by polarization measurements using STAR at RHIC. ANL physicists have worked on a number of related tasks over the past 8-9 years to achieve this goal. For example, the Argonne spin physics group has had the primary responsibility for upgrading and operating the polarimeter for the AGS, which is the injector to RHIC. In the past, a number of dedicated AGS runs have improved the proton beam polarization at the highest AGS energies. However, during the recent commissioning of polarized beam at RHIC, a lower than anticipated polarization was measured in the AGS. A variety of explanations were proposed, especially at low energies, but they were shown incapable of reproducing all the measurements. After an extensive analysis of AGS polarimeter data from past runs, it was concluded that at low energies either there were problems with the polarimeter operation, or the beam polarization in the AGS was much lower than previously achieved. These results were written in a technical paper (STAR and BNL C-AD notes).

The RHIC absolute polarization is presently tied to an AGS experiment, E925, because a pp elastic scattering polarimeter was in the extracted polarized proton beam as part of E925. This experiment was performed by a collaboration including our group, and it was led by A. Yokosawa from ANL. (For the RHIC calibration, it must be assumed that the beam polarization in the AGS and at the E925 apparatus was the same, even though the beam was heavily collimated before reaching the E925 polarimeter.) Charged pion asymmetries at $22 \mathrm{GeV} / \mathrm{c}$ off hydrogen and carbon targets were measured in E925 with a magnetic spectrometer. Partial results of this experiment have been published. The final paper was begun with significant work by C. Allgower of ANL and others, and is now being completed by two other ANL physicists. The results from E925 are compared to those from FNAL E704 at $200 \mathrm{GeV} / \mathrm{c}$ in Fig. 1; the latter data are also from an experiment led by A. Yokosawa. Large asymmetries are observed at both momenta. This suggests large asymmetries will persist to still higher energies, making inclusive charged pion production an attractive reaction for a polarimeter at a high energy proton accelerator or collider, such as RHIC or HERA. An approximate mirror symmetry between $\pi^{+}$and $\pi^{-}$is observed as a function of $x_{F}$. These data are reminiscent of the hyperon polarization results at high energy, although 
the exact physical connection (if any) is not understood. The E925 experiment also found that the asymmetries measured with hydrogen, carbon, and $\mathrm{CH}_{2}$ targets were essentially identical, as shown in Fig. 2. This result was unexpected, because it contradicts the prediction obtained when the reaction on carbon is modeled by multiple scattering off nucleons in the nucleus.

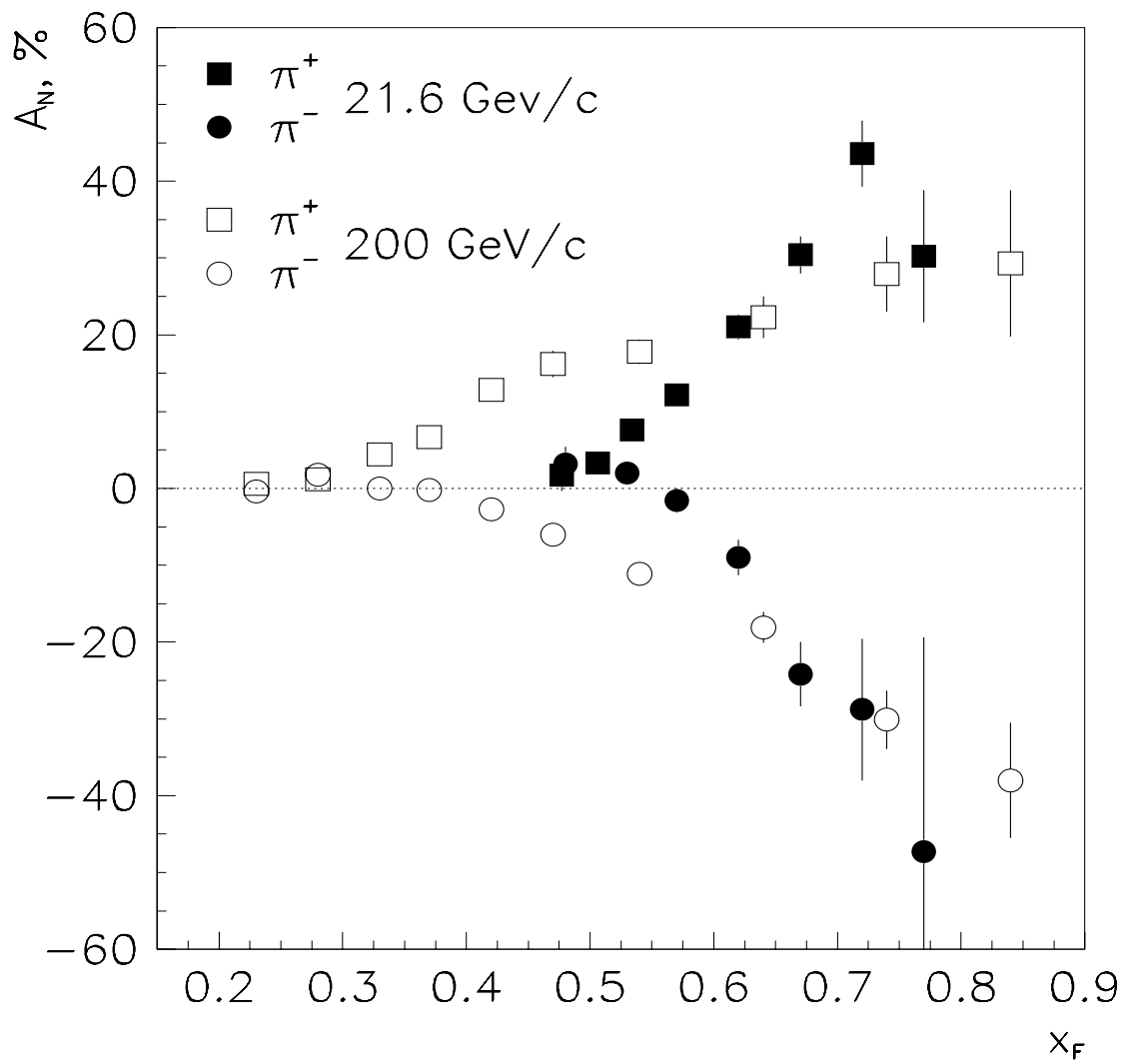

Figure 1: Comparison of inclusive analyzing powers $A_{N}$ for $\pi^{+}$and $\pi^{-}$from carbon at $21.6 \mathrm{GeV} / \mathrm{c}$ from E925 and hydrogen at $200 \mathrm{GeV} / \mathrm{c}$ from FNAL E704 as a function of $x_{F}$. 


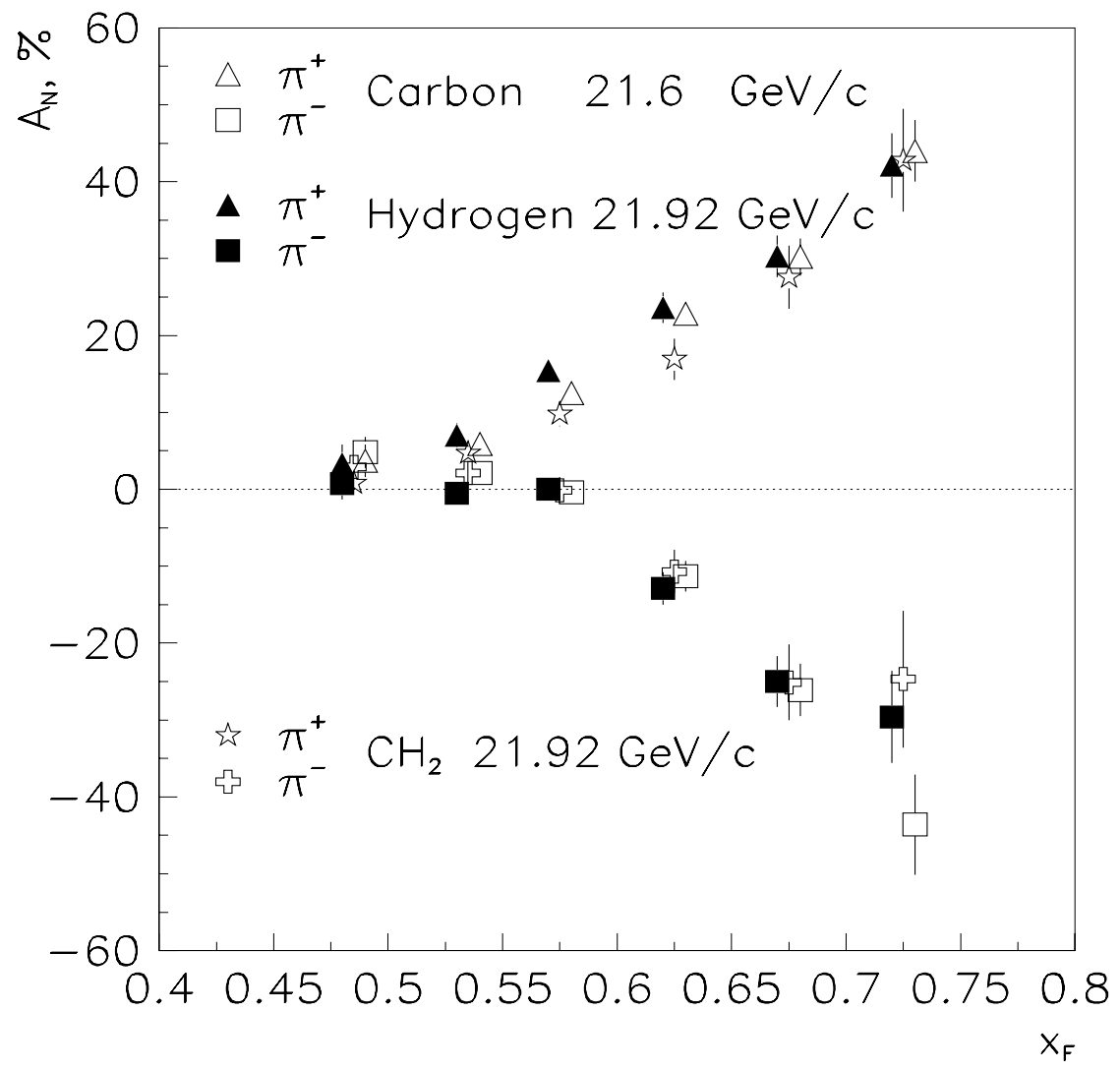

Figure 2: Analyzing powers for $\pi^{+}$and $\pi^{-}$as a function of $x_{F}$ on carbon, $\mathrm{CH}_{2}$, and hydrogen.

Note some points are slightly offset from the true value of $x_{F}$ to make it easier to distinguish the points.

The primary focus of the STAR experiment is to study the properties of nuclear matter at high temperature and density using collisions of gold nuclei at energies up to $\sqrt{s}=200 \mathrm{AGeV}$. It is anticipated that these collisions will lead to the formation of a quarkgluon plasma (QGP), an extended system in which quarks and gluons are no longer confined in hadrons. At this time, however, there is no theoretical agreement on what would constitute an unambiguous QGP signal. In the summer of 2000, Au-Au collisions at $\sqrt{s}=130 \mathrm{AGeV}$ were studied at RHIC. This produced several papers during the first half of 2001. Two papers were published (STAR Collaboration, K. H. Ackermann et al., Phys. Rev. Lett. 86402 (2001) and STAR Collaboration, C. Adler et al., Phys. Rev. Lett. 864778 (2001)) and two more were submitted. A fifth paper was completed and made available to the other RHIC collaborations in the last week of June 2001, notifying them that it would be submitted in the first week of July. Measurements of elliptic flow are described in the first and fifth papers; elliptic flow refers to the azimuthal anisotropy in particle emission observed when the two nuclei collide off-center. The second paper reports measurements of the midrapidity antiproton-to-proton ratio, which at $130 \mathrm{AGeV}$ is significantly higher than the CERN measurements at 17.4 AGeV, as shown in Fig. 3. The third paper discussed the production of 
negative hadrons. The use of Hanbury Brown-Twiss interferometry to measure the size of the pion-emitting region in the nuclear collision was described in the fourth paper.

Two papers from the Crystal Ball experiments were also submitted for publication ("Measurement of $K^{-} p \rightarrow \eta \Lambda$ Near Threshold," A. Starostin et al. and "Determination of the Quadratic Slope Parameter in $\eta \rightarrow 3 \pi^{0}$ Decay," W. B. Tippens et al.). The first is a major paper describing analysis of kaon beam data at beam momenta of 720 and $750 \mathrm{MeV} / \mathrm{c}$, and the second is a letter on pion beam data at $720 \mathrm{MeV} / \mathrm{c}$. A third paper was published ("A Measurement of Neutron Detection Efficiencies in NaI Using the Crystal Ball Detector," T. D. S. Stanislaus et al., Nucl. Instrum. Meth. Phys. Res. $\mathbf{A 4 6 2} 463$ (2001)). A fourth paper is nearly ready to be submitted for publication.

Finally, a paper on pp elastic scattering spin measurements at Saclay, described in the last semiannual report, was accepted for publication in Phys. Rev. C. There is a possibility that one of the Saclay collaborators will write one final paper on this work, but otherwise this program will be complete.

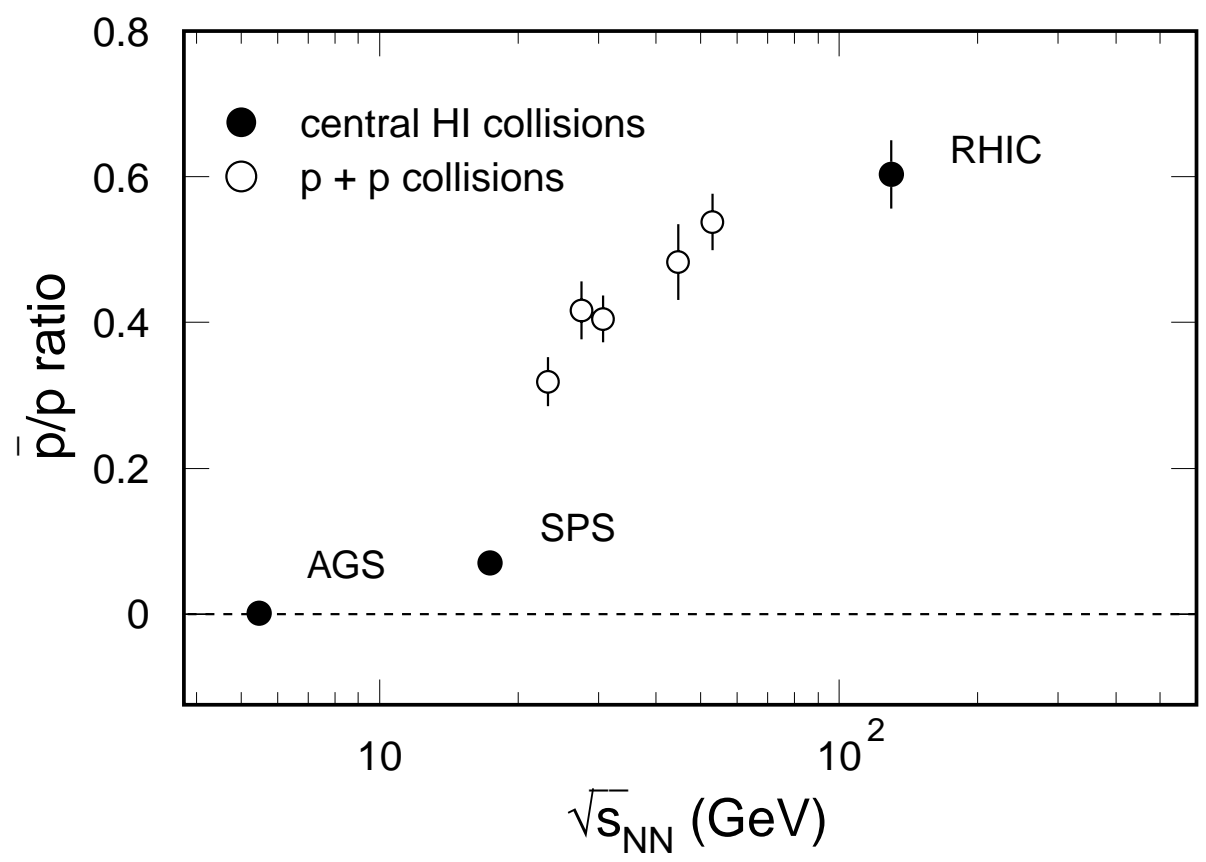

Figure 3: Midrapidity antiproton-to-proton ratio $(\bar{p} / p)$ measured in central heavy-ion collisions (filled symbols) and elementary $p+p$ collisions (open symbols). Errors, either shown or smaller than the symbol size, are statistical and systematic errors added in quadrature.

(H. M. Spinka and R. Cadman) 


\section{I.A.2 Collider Detector at Fermilab}

\section{a) Physics Results}

The emphasis of the collaboration has largely shifted to getting the detector going for Run 2. Some Run 1 analyses remain to be completed and submitted for publication. Will Bell is continuing to progress on his thesis analysis limit on the branching fraction for $B_{s} \rightarrow \psi \pi^{\prime}$; this is largely a feasibility study for Run 2. Bob Blair continues as QCD physics convener, where a few analyses remain pending; some of the photon searches are getting out. Steve Kuhlmann with students has prepared the photon muon and inclusive photon analyses for publication. The composition of the photon muon sample in terms of heavy flavor is determined by studying the transverse momentum of the muon with respect to the jet associated with it $\left(P_{T}^{r e l}\right)$ as illustrated in Figure 1, and the cross section, with predictions, in Figure 2. The inclusive photon cross section, discrepancy between theory and data as a function of scaled energy $\left(x_{T}\right)$ is shown in Figure 3 for data at $\sqrt{s}$ of 630 and $1800 \mathrm{GeV}$.

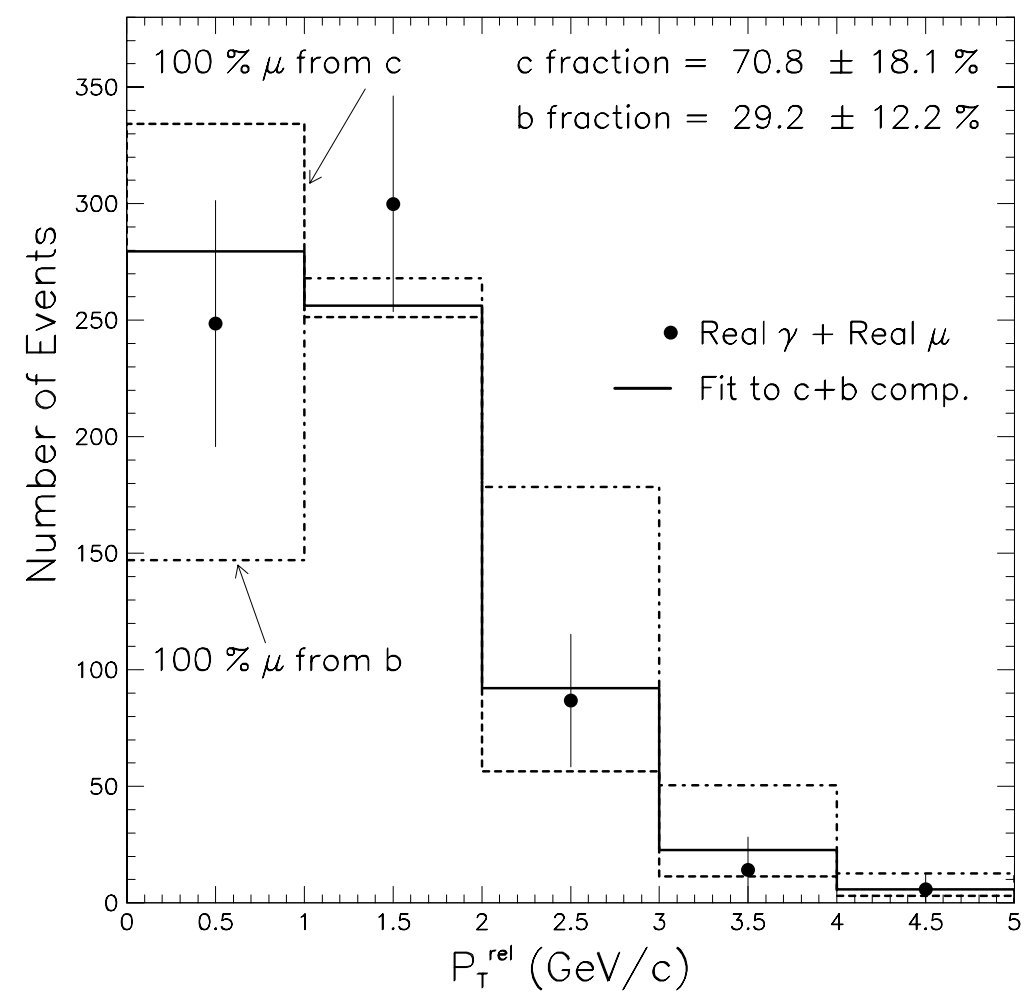

Figure 1: Heavy flavor composition of the photon plus muon data is determined by the muon momentum relative to the jet axis. 


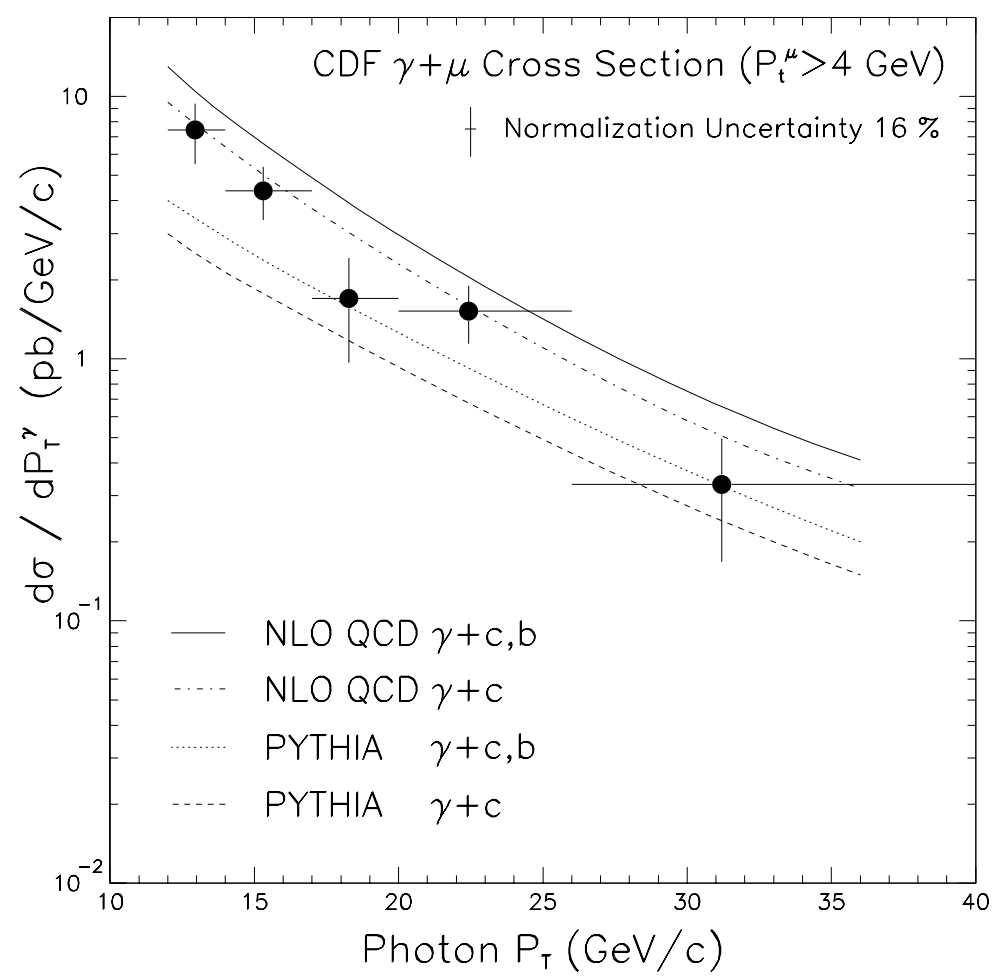

Figure 2: Cross section for photon plus muon production

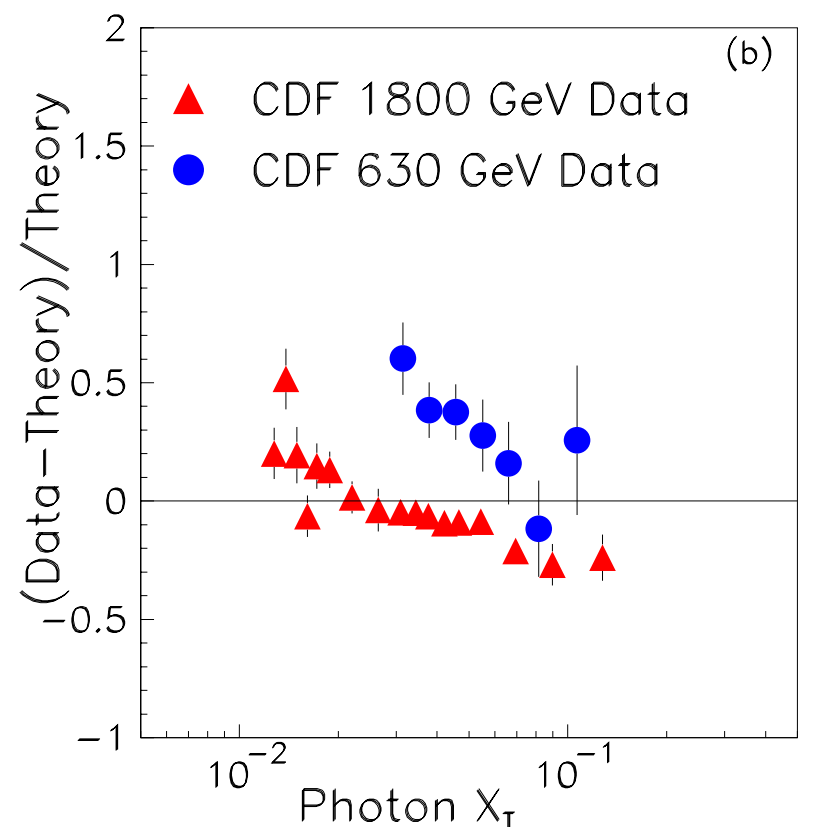

Figure 3: Cross section discrepancy between theory and data for inclusive photons from $\bar{p} p$ data at 1800 and $630 \mathrm{GeV}$, plotted in $x_{T}$. 


\section{b) The CDF Run 2 Commissioning}

Shower max calorimeter readout continues to be a major project for us; Karen Byrum is project manager and Gary Drake is Chief Engineer, and John Dawson has taken on several of the components. All of the multiple card components have been installed and operated. The preamplifier design has been iterated with tests on the detector .Jimmy Proudfoot, Steve Kuhlmann, Karen Byrum, along with Larry Nodulman; continue to develop software to handle the hardware within the B0 online system. Karen continues working with Gary and Mike Lindgren to develop the plan needed to reach a stable operating configuration. A problem has been seen in that in about $10 \%$ of channels for the shower max chambers, preamps are repeatedly burned out, presumably due to micro-discharges. Diode protection schemes are being developed.

The CDF detector was rolled into the hall in April, with silicon in and full drift chamber instrumentation. First beam came in May. Drift chamber and central calorimeter commissioning have gone fairly well. The central EM occupancy, phototube ratio and rms of the ratio are shown in Figure 4 for minimum bias events. A few cable swaps and bad tubes are apparent. The shower max wire chamber occupancy and pedestal are shown in Figure 5. One whole chamber is off due to digital electronics problems, and the two blanks in the second from the rightmost chamber were traced to a high voltage cabling problem, and the other bad regions are due to the preamp burn out problem.

\section{I.A.3. The CDF Upgrade Project}

\section{a) Run II Planning}

The Run 2 physics workshops, other than B physics where Barry Wicklund continues as a principal, are largely completed and written up. Steve Kuhlmann has taken a leading role in QCD issues as well as optimizing b dijet mass resolution for Higgs searches.

Thanks to a few four jet events at Aleph, the Higgs search has become the flagship analysis of Run 2 and the only officially allowed motivator for high luminosity Run $2 b$ upgrades. Steve Kuhlmann, along with Joey Huston, has taken charge of replacing the preradiator chambers for Run $2 \mathrm{~b}$. A design using MINOS scintillator cuttings with recycled electronics is being explored. 

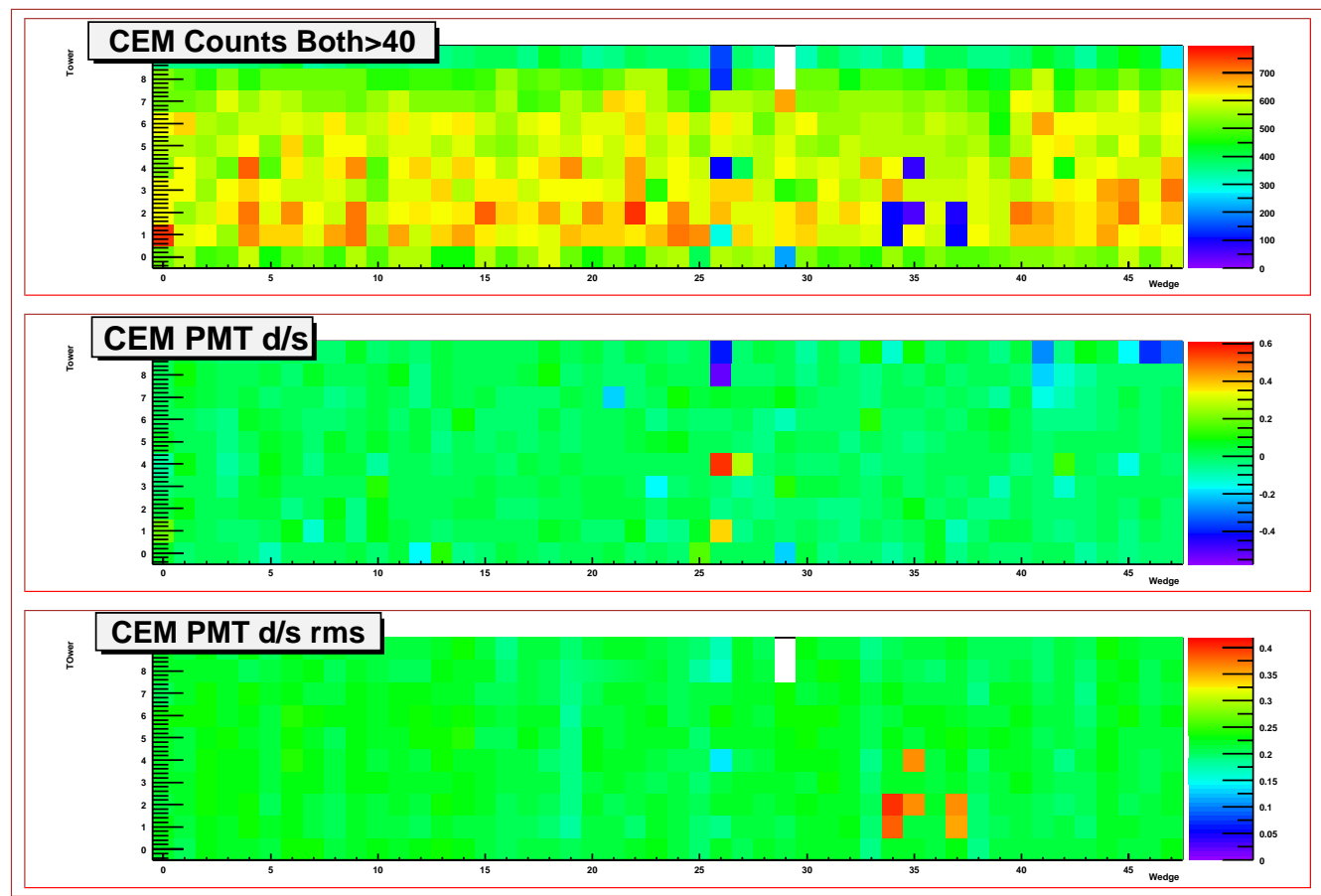

Figure 4: Central EM calorimeter has 48 wedges of 10 towers. Temperature plots of occupancy with both PMTs on, PMT ratio, and rms of the PMT ratio as shown. Cable mismatch makes for low occupancy and high rms. Wedge 5E (29) has two fewer towers.

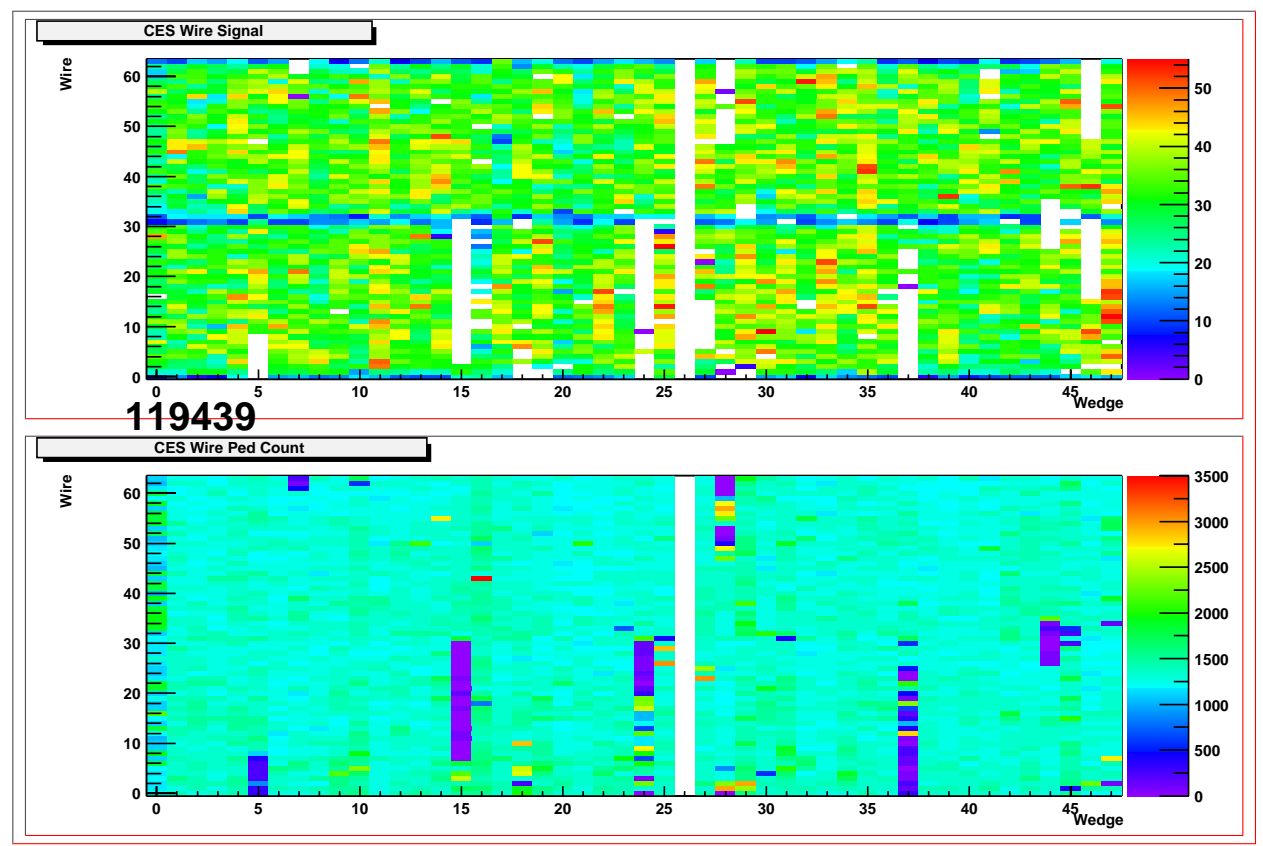

Figure 5: Temperature plots show wire channel signal occupancy for the 32 channels per wedge, and pedestal count with zero suppression at about $\pm 1 \sigma$. Burned out preamps give smaller rms implying fewer pedestal counts. Wire channels $0,31,32$ and 63 are single wires, the rest are pairs. 
Karen Byrum, Steve Kuhlmann, Bob Blair, Masa Tanaka, and John Dawson and company continue working with the Michigan group to integrate the Level 2 trigger hardware for shower max and calorimeter isolation. General Level 2 problems tend to dominate.

Bob Wagner continues developing offline software for calorimeter reconstruction with emphasis on electron code. Bob, Steve and Barry Wicklund are involved in code for the wire chamber data reconstruction.

Larry and Barry are physics group delegates to the trigger dataset working group for top/electroweak and b physics respectively. Karen has become one of four heads of the calorimeter group.

(L. J. Nodulman)

\section{I.A.4 Non-Accelerator Physics at Soudan}

In July 2001, the Soudan-2 experiment completed the taking of data using its finegrained iron tracking calorimeter of total mass 963 tons. The total data exposure was 5.91 fiducial kiloton-years (kTy). Results presented here are based upon a $5.1 \mathrm{kTy}$ exposure.

Topologies for contained events in Soudan 2 include single track and single shower events (mostly $v_{\mu}$ and $v_{\mathrm{e}}$ quasi-elastic reactions) and multiprong events. Flavor-tagging proceeds straightforwardly: An event having a leading, non-scattering track with ionization $\mathrm{dE} / \mathrm{dx}$ compatible with muon mass is a candidate $v_{\mu}$ charged current $(\mathrm{CC})$; an event having a prompt, relatively energetic shower prong is a candidate $v_{\mathrm{e}} \mathrm{CC}$ event. Recoil protons of momenta greater than approx. $350 \mathrm{MeV} / \mathrm{c}$ are imaged by the calorimeter, allowing a muchimproved measurement of the incident neutrino direction, especially for sub-GeV quasielastic reactions.

We measure the atmospheric neutrino flavor ratio-of-ratios $\mathrm{R}$ using single track and single shower events which are fully contained within the calorimeter (all hits more than 20 $\mathrm{cm}$ from the nearest surface). These samples contain mostly quasi-elastic neutrino reactions, but include a background of photon and neutron reactions originating in cosmic ray muon interactions in the surrounding cavern rock. The latter "rock events" are mostly tagged by coincident hits in the active shield, however some are unaccompanied by shield hits and constitute a background. The amount of zero-shield-hit rock background in a neutrino event sample is estimated by fitting event vertex-depth distributions to a combination of taggedrock and $v$ Monte Carlo distributions. As expected, the fits show the background to be mostly confined to outer regions of the calorimeter.

The track and shower event samples for our 5.1 kTy exposure are as follows: 133 raw data tracks, 193 raw data showers, 1097 Monte Carlo Tracks, 1017 Monte Carlo 
showers. The data background subtracted results are $105.1 \pm 12.7$ tracks and $142.3 \pm 15.6$ showers. Our full detector Monte Carlo simulation of atmospheric neutrino interactions is based on the 1996 Bartol flux for the Soudan site. After correction for cosmic ray muon induced background, the number of single track events observed in data is less than the number of single shower events, whereas the null oscillation Monte Carlo predicts the relative rates to be other-way-round. Consequently the flavor ratio-of-ratios obtained is less than 1.0 and is anomalous: $\mathrm{R}=0.68 \pm 0.11$ (stat) \pm 0.06 (sys).

The phenomenology for $v_{\mu}$ to $v_{\tau}$ oscillations is quite specific; neutrinos of muon flavor can "disappear" according to the equation

$$
\left.\mathrm{P}\left(\mathrm{v}_{\mu} \rightarrow \mathrm{v}_{\tau}\right)=\sin ^{2}(2 \theta) \sin ^{2}(1.27) \Delta \mathrm{m}^{2}\left[\mathrm{eV}^{2}\right] \mathrm{L}[\mathrm{km}] / \mathrm{E}[\mathrm{GeV}]\right)
$$

Consequently it is optimal to analyze for neutrino oscillations using the variable $\mathrm{L} / \mathrm{E}$. With the Soudan 2 calorimeter, measurement of event energy for charged current reactions is straightforward; we do this with resolution $\Delta \mathrm{E} / \mathrm{E}$ which is $20 \%$ for $v_{\mu} \mathrm{CC}$ 's and $23 \%$ for $v_{\varepsilon}$ CC's. To determine the neutrino path length $\$ L \$$ however, the zenith angle $\theta_{z}$ of the incident neutrino must be reconstructed with accuracy. The path length can be calculated from the zenith angle knowing the Earth's radius, the depth of the detector, and the mean neutrino production height. The latter is a function of $v$ flavor, $v$ energy, and zenith angle. We select from our data an event sample suited to this measurement. We use a quasi-elastic track or shower event provided that the recoil proton is measured and that $\mathrm{P}_{\text {lept }}$ exceeds $150 \mathrm{MeV} / \mathrm{c}$; otherwise, if the recoil nucleon is not visible, we require the single lepton to have $\mathrm{E}_{\mathrm{vis}}$ great than $600 \mathrm{MeV}$. We also select multiprong events, provided they are energetic $\left(\mathrm{E}_{\mathrm{vis}}\right.$ greater than $700 \mathrm{MeV}$ ) and have vector sum of $\mathrm{P}_{\mathrm{vis}}$ exceeding, the final state lepton momenta are required to exceed $250 \mathrm{MeV} / \mathrm{c}$. For the selected sample, flavor tagging is estimated to be correct for more than $92 \%$ of events. The resolution for recovering the incident neutrino direction is evaluated using the mean angular separation between "true" versus reconstructed neutrino direction in Monte Carlo events. The mean separations are $33.2^{\circ}$ for $v_{\mu} \mathrm{CC}$ 's and $21.3^{\circ}$ for $v_{\mathrm{e}} \mathrm{CC}$ 's . The resolution in $\log (\mathrm{L} / \mathrm{E})$ ( $\mathrm{L}$ in kilometers, $\mathrm{E}$ in $\mathrm{GeV}$ ) is better than 0.5 for the selected sample. Hereafter we refer to these events as "HiRes events".

The zero-shield-hit rock background, as estimated by the fits to event vertex depth distributions, comprises $6.8 \%(5.1 \%)$ of the $v_{\mu}\left(v_{\mathrm{e}}\right)$ flavor sample of HiRes events.

The table shows the HiRes event populations. After background subtraction there are 106.3 data events of muon flavor and 132.8 events of electron flavor. Using these events, whose mean energy is higher than that of our track and shower events, the ratio-of-ratios is $\mathrm{R}=0.67 \pm 0.12$, which is also significantly less than 1.0 .

$\begin{array}{lcc} & v_{\mu} & \nu_{\mathrm{e}} \\ \text { Data raw } & 114.0 \pm 10.7 & 140.0 \pm 11.8 \\ \text { Data, subt. } & 106.3 \pm 14.7 & 132.8 \pm 13.4 \\ \text { Monte Carlo } & 158.5 \pm 4.8 & 132.8 \pm 4.4\end{array}$


The atmospheric Monte Carlo (MC) sample represents 28.2 kiloton years of exposure. The $\mathrm{MC}$ event rates have been normalized to the $v_{\mathrm{e}}$ data sample. The assumption implicit with this adjustment is that the $v_{\mathrm{e}}$ sample is devoid of oscillation effects. The figure below shows HiRes distributions with this normalization in place. The distributions of these samples in cosine of the zenith angle are shown in the figure. For $v_{\mathrm{e}}$ events, the shape of the distribution coincides with that predicted by the Monte Carlo for null oscillations. The distribution of $v_{\mu}$ data however, falls below the MC prediction in all bins with the relative dearth being more pronounced for $v_{\mu}$ 's incident from below horizon. Distributions in $\log (\mathrm{L} / \mathrm{E})$ for HiRes events are shown in the next figure. For null oscillations this variable distributes according to a 'phase space' which reflects the neutrino points-of-origin throughout the spherical shell volume of the Earth's atmosphere. That is, down-going v's populate the peak at $\operatorname{lower} \log (\mathrm{L} / \mathrm{E})$ from 0.0 to 2.0. Neutrinos incident from/near the horizon occur within the dip region extending from 2.0 to 2.6, while upward-going neutrinos populate the peak at higher values. Allowing for statistical fluctuations, the $v_{\mathrm{e}}$ data follows the shape of the null oscillation MC distribution. In contrast, the $v_{\mu}$ data falls below the null oscillation MC for all but the most vertically down-going flux.
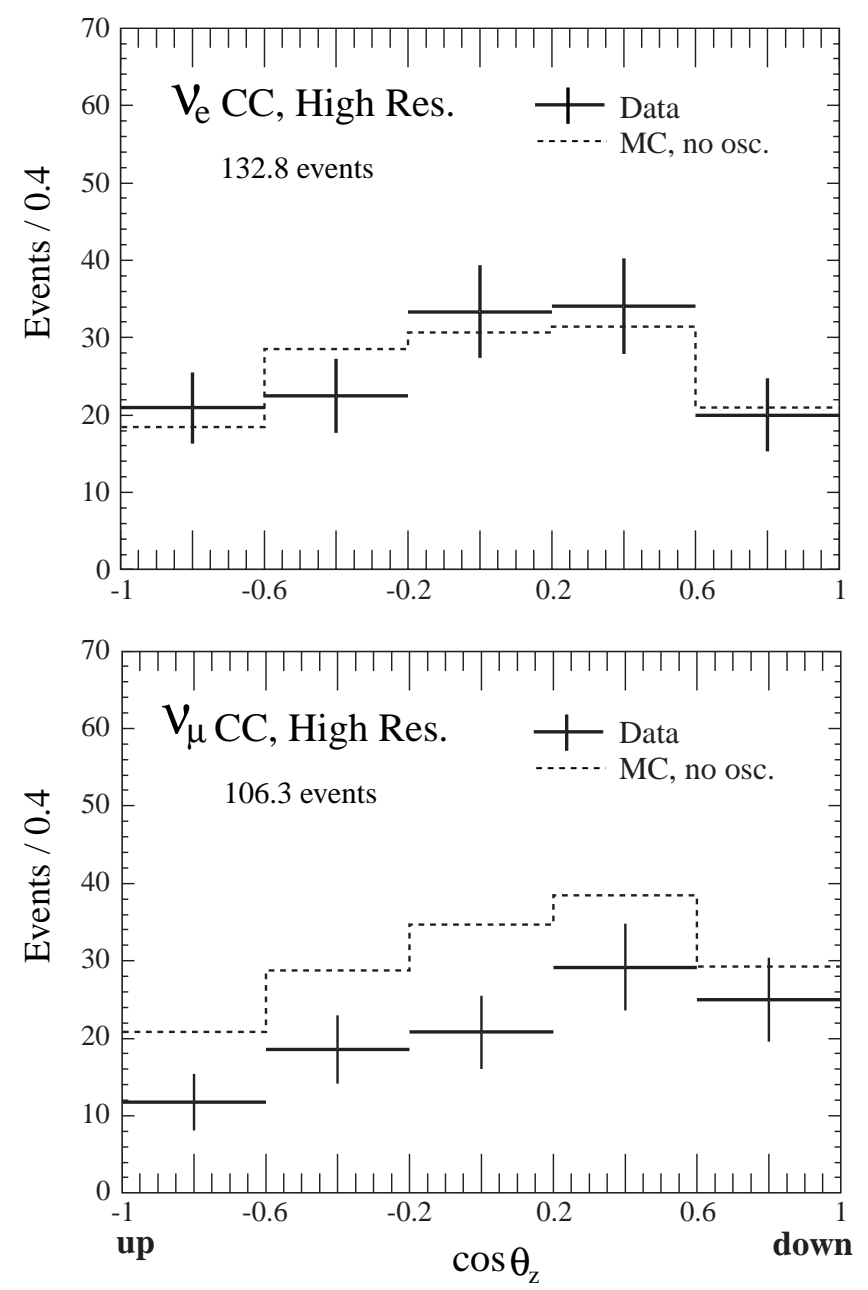

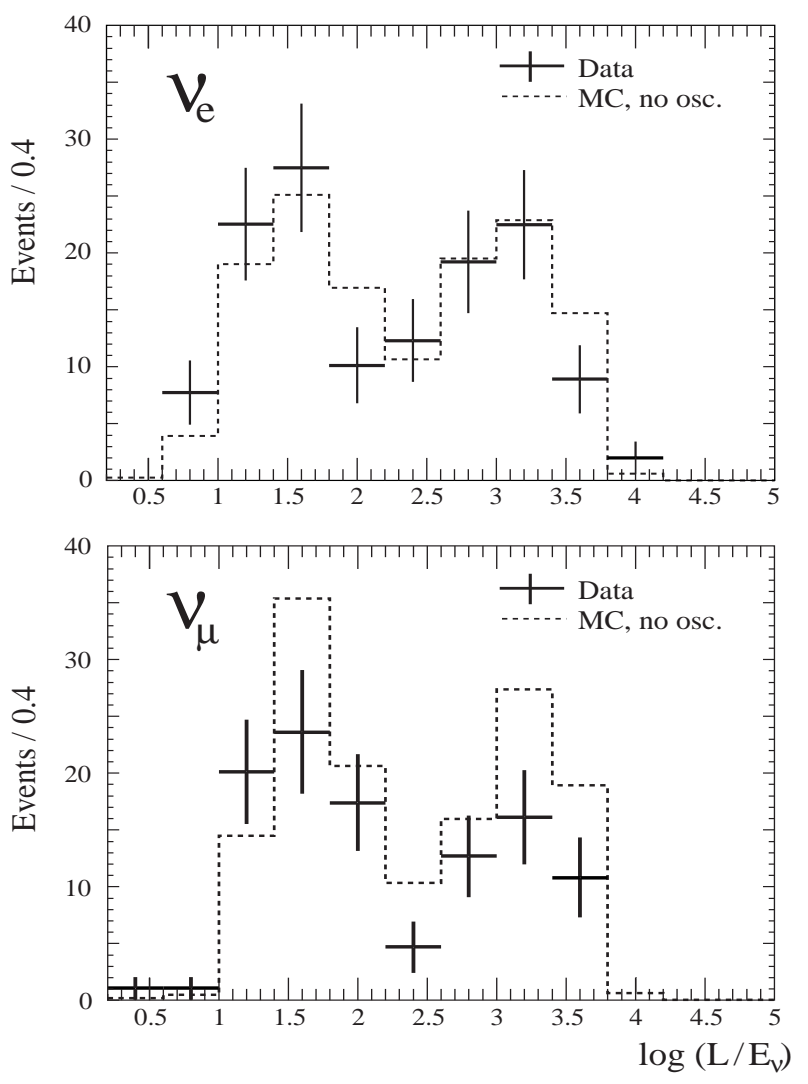

To convert results of our atmospheric neutrino simulation generated under the nooscillation hypothesis into simulated neutrino oscillation data, we apply to every MC event an L/E-dependent weight representing the probability of muon flavor survival for a given $\Delta \mathrm{m}^{2}$ and $\sin ^{2}(2 \theta)$.

To determine the neutrino oscillation parameters $\Delta \mathrm{m}^{2}$ and $\sin ^{2}(2 \theta)$ from our data, we construct a $\chi^{2}$ function over the plane-of-parameters. For points $i, j$ ) in the physical region of the $\sin ^{2}(2 \theta)-\log \left(\Delta \mathrm{m}^{2}\right)$ plane, we fit the MC expectation to our data at each point. The MC flux normalization, $f_{v}$ as well as $\sin ^{2}(2 \theta)$ and $\log \Delta \mathrm{m}^{2}$, is a free parameter:

$$
\left(\chi_{\text {data }}^{2}\right)_{i j}=\chi^{2}\left(\sin ^{2}(2 \theta), \Delta \mathrm{m}^{2}, \mathrm{f}_{\mathrm{v}}\right)=\sum_{\mathrm{k}=1}^{8}\left[\mathrm{~N}_{\mathrm{k}}(\text { data-bkgd })-\mathrm{f}_{\mathrm{v}} \mathrm{N}_{\mathrm{k}}(\mathrm{MC})\right]^{2} / \sigma_{\mathrm{k}}^{2}
$$

We assume that the oscillation affecting our data is purely $v_{\mu}$ into $v_{\tau}$ and that the $v_{\mathrm{e}}$ data is unaffected.

The $\chi 2$ is summed over data bins containing our selected (HiRes) $v_{\mu}$ and $v_{\mathrm{e}}$ samples, where $\mathrm{k}=1-7$ are $v_{\mu} \log (\mathrm{L} / \mathrm{E})$ bins, with $\mathrm{k}=8$ containing all the $\mathrm{v}_{\mathrm{e}}$ events. The denominator $\sigma\{k\}^{2}$ accounts for finite statistics in the neutrino Monte Carlo and for uncertainty in the rock background in the $\$ \backslash n u \$$ data. Not yet included are error terms which address systematic errors in the analysis, however preliminary examination shows statistical errors to be the 
dominant error source in the analysis. The $M C$ counts $\mathrm{N}_{\mathrm{k}}(\mathrm{MC}) \$$ for the $\mathrm{k}^{\text {th }}$ bin are constructed using oscillation weight factors.

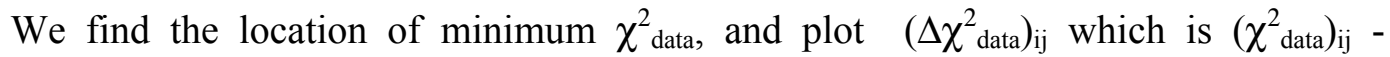
$\left(\chi_{\text {data }}^{2}\right)_{\min }$. A crater region of low $\chi^{2}$ values is discerned, at the bottom of which is a relatively flat basin. The lowest point $\chi^{2}{ }_{\text {min }}$ occurs at values $\sin ^{2}(2 \theta)=0.90, \Delta \mathrm{m}^{2}=7.910^{-3}$, with flux normalization $\mathrm{f}_{v}=0.78$.

An additional structure is the $\Delta \chi^{2}$ ridge which occurs at large mixing angle and for $\Delta \mathrm{m}^{2}$ above $10^{-2} \mathrm{eV}^{2}$. For oscillation solutions in this regime, depletion in the downwardgoing $v_{\mu}$ neutrino flux with sub-GeV energies is predicted for $v_{\mu}$ to $v_{\tau}$ oscillations arising from the first oscillation minimum. Our HiRes events have sufficient resolution to show such an effect if it would be present. However, no pronounced depletion is observed, and so the $\chi^{2}$ has a high value there.

To find the region allowed for the oscillation parameters by our data at $90 \%$ confidence level (CL), we use the method of Feldman and Cousins. At each of 2500 points $(\mathrm{i}, \mathrm{j})=\sin ^{2}(2 \theta)_{\mathrm{i}}, \Delta \mathrm{m}_{\mathrm{j}}^{2}$ on a grid spanning the physical region of the plane parameters, we run 1000 simulated experiments. For each of the simulated sets, we find $\Delta \chi_{\mathrm{ij}}^{2}$ such that $\left(\Delta \chi_{\text {sim }}^{2}\right)_{\mathrm{ij}}$ is less than for $90 \%$ of the simulated experiments at $(\mathrm{i}, \mathrm{j})$. The surface defined by local $\left(\Delta \chi_{90}^{2}\right)_{\mathrm{ij}}$ over the oscillation parameters plane is then plotten. Note that the surface is not a plane at $\left(\Delta \chi^{2}{ }_{90}\right)=4.61$, but rather has a concave shape. The central shaded portion is approximately $\Delta \chi^{2}=4.6$, however the outlying regions have $\Delta \chi^{2}$ values which are lower. At each point over the physical region, if $\Delta \chi^{2}$ (data)ij is less than $\left(\Delta \chi^{2}{ }_{90}\right)_{\mathrm{ij}}$ then $(\mathrm{i}, \mathrm{j})$ belongs to the allowed region of the $90 \%$ CL contour.

The region allowed by our data at $90 \%$ CL is shown by the shaded area in the figure below. Although the $\chi^{2}$ minimum occurs at the location depicted by the solid circle, the relatively flat basin of our $\Delta \chi^{2}$ surface extends to lower $\Delta \mathrm{m}^{2}$ values. 


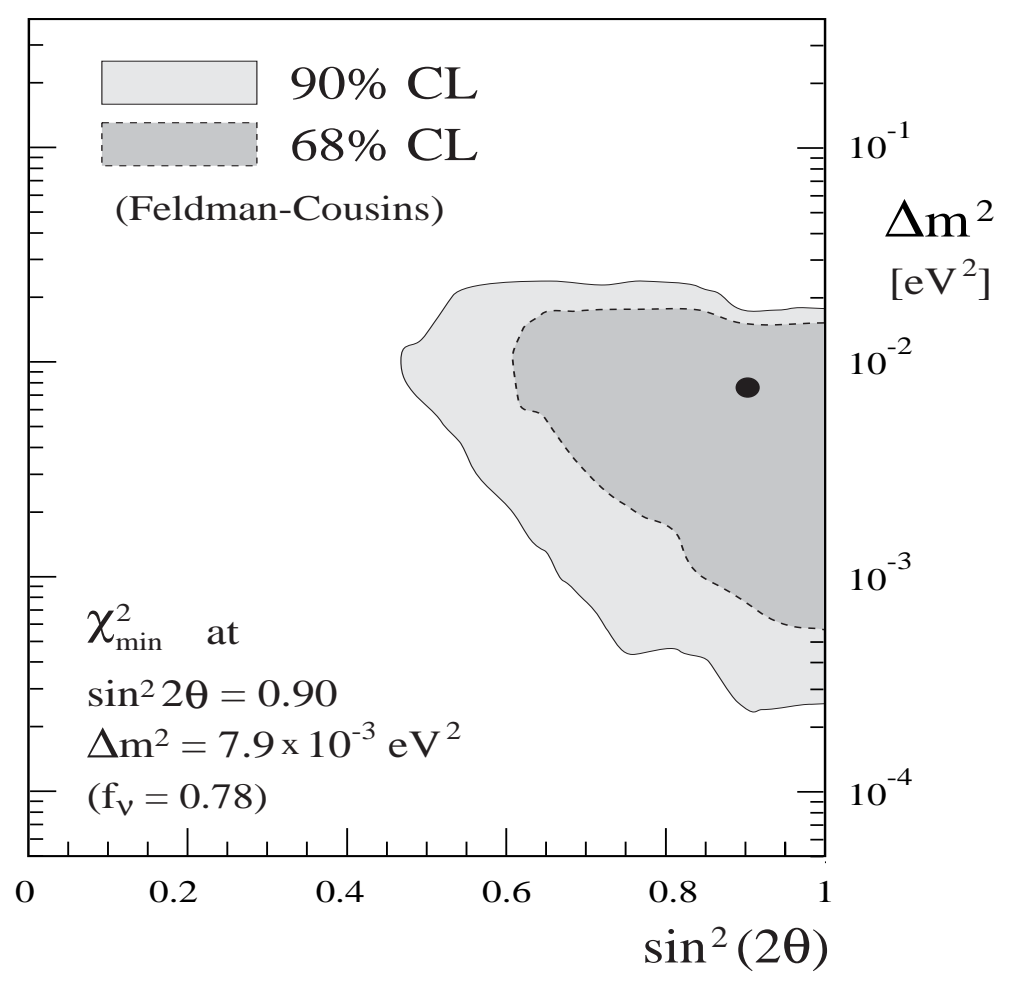

(M. C. Goodman)

\section{I.A.5. ZEUS Detector at HERA}

\section{(a) Physics Results}

Five papers were published in this period and three more manuscripts were submitted for publication. In the following, we shall summarize some of the papers published in this period of time.

i) Measurement of Dijet Production in Neutral Current Deep Inelastic Scattering at High $Q^{2}$ and Determination of $\alpha_{s}$

Dijet production has been studied in neutral current deep inelastic $e^{+} p$ scattering for $470<\mathrm{Q}^{2}<20000 \mathrm{GeV}^{2}$. Dijet differential cross sections were measured in a kinematic region where both theoretical and experimental uncertainties are small. Next-to-leading order QCD calculations describe the measured differential cross sections well. A QCD analysis of the measured dijet fraction as a function of $\mathrm{Q}^{2}$ allows both a test of the energy-scale dependence of the strong coupling constant and a precise determination of $\alpha_{S}\left(M_{Z}\right)$. The value of $\alpha_{S}\left(M_{Z}\right)$, as determined from the QCD fit, is $\alpha_{S}\left(\mathrm{M}_{Z}\right)=0.1166 \pm 0.0019$ (stat.) ${ }_{-0.0033}^{+0.0024}$ (exp.) ${ }_{-0.0044}^{+0.0057}$ (theor.). Figure 1 shows the $\alpha_{S}(\mathrm{Q})$ values determined from the QCD fit as a 
function of $Q$. Over a large range in $Q$ the values are in excellent agreement with the predicted running of the strong coupling constant.

\section{ZEUS}

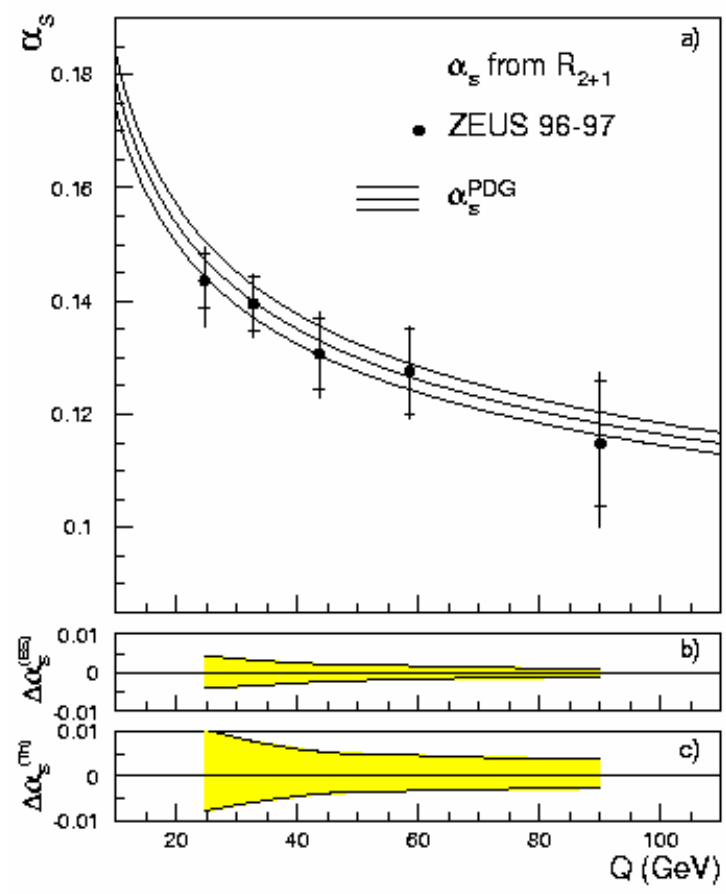

Figure 1. a) The $\alpha_{\mathrm{S}}(Q)$ values determined from the QCD fit of the measured dijet fraction as a function of $Q$. The three curves indicate the renormalization group predictions obtained from the PDG $\alpha_{S}\left(M_{Z}\right)$ value and its associated uncertainty. B) The uncertainty on $\alpha_{\mathrm{S}}$ due to the absolute energy scale of the jets. C) The total theoretical uncertainty associated with this determination of $\alpha_{S}$.

\section{ii) Multiplicity Moments in Deep Inelastic Scattering at HERA}

Multiplicity moments of charged particles in deep inelastic scattering have been measured. The moments for $Q^{2}>1000 \mathrm{GeV}^{2}$ were studied in the current region of the Breit frame. The evolution of the moments was investigated as a function of restricted regions in polar angle and, for the first time, both in the transverse momentum and in absolute momentum of the final-state particles. Analytic perturbative QCD predictions in conjunction with the hypothesis of Local Parton-Hadron Duality (LPHD) reproduce the trends of the moments in polar-angle regions, although some discrepancies are observed. For the moments restricted either in transverse or absolute momentum, the analytic results combined with the LPHD hypothesis show considerable deviations from the measurements, see Figure 2. The study indicates a large influence of the hadronisation stage on the multiplicity distributions in the restricted phase-space regions studied here, which is inconsistent with the expectations of the LPHD hypothesis. 


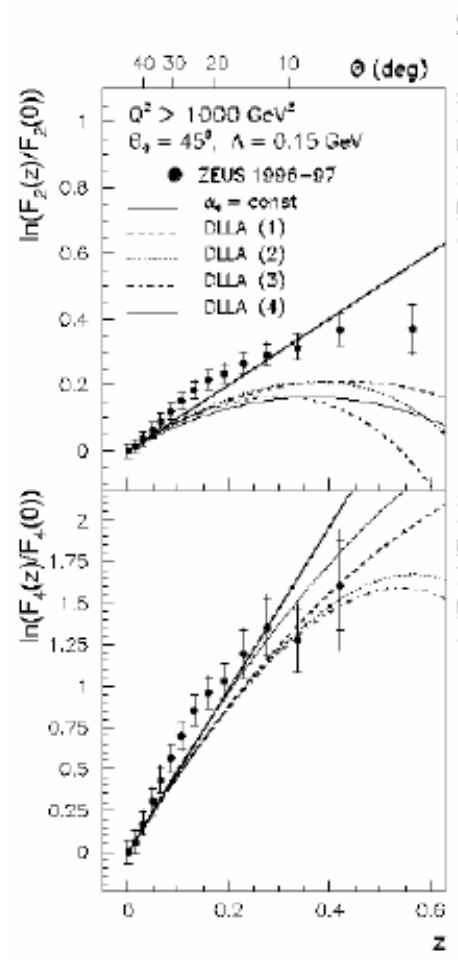

ZEUS

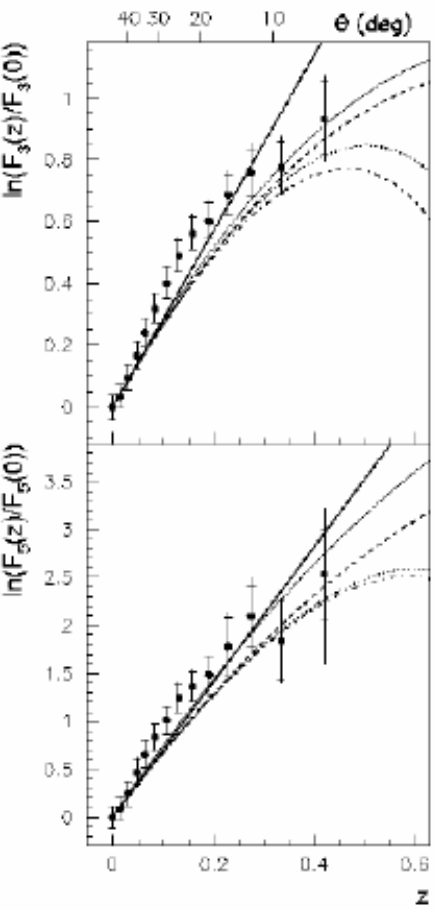

Figure 2: Normalized factorial moments as a function of the scaling variable $z$ for $\theta_{0}=45^{\circ}$ and $\Lambda=0.15 \mathrm{GeV}$, compared to various DLLA QCD predictions.

iii) Measurement of Open Beauty Production in Photoproduction at HERA

The production and semi-leptonic decay of heavy quarks have been studied in the photoproduction process $e^{+} p \rightarrow e^{+}+$dijet $+\bar{e}+X$. Events with photon-proton center-ofmass energies between 134 and $269 \mathrm{GeV}$ and a photon virtuality less than $1 \mathrm{GeV}^{2}$ were selected requiring at least two jets of transverse energy greater than 7(6) $\mathrm{GeV}$ and an electron in the final state. The electrons were identified by employing the ionization energy loss measurement. The contribution of beauty quarks was determined using the transverse momentum of the electron relative to the axis of the closest jet. The data, after background subtraction, were fit with a Monte Carlo simulation including beauty and charm decays. The measured beauty cross section was extrapolated to the parton level with the $b$ quark restricted to the region of transverse momentum $p_{T}^{b}>5 \mathrm{GeV}$ and pseudorapidity $\left|\eta^{b}\right|<2$. The extrapolated cross section is $1.6 \pm 0.4$ (stat.) ${ }_{-0.5}^{+0.3}$ (syst.) ${ }_{-0.4}^{+0.2}$ (ext.) nb. In Figure 3 the result is compared to a perturbative QCD calculation performed to next-to-leading order. The extrapolated cross section lies somewhat above the central NLO prediction, consistent with the general observation that NLO QCD calculations underestimate beauty production both in hadroproduction and photoproduction. 


\section{ZEUS}

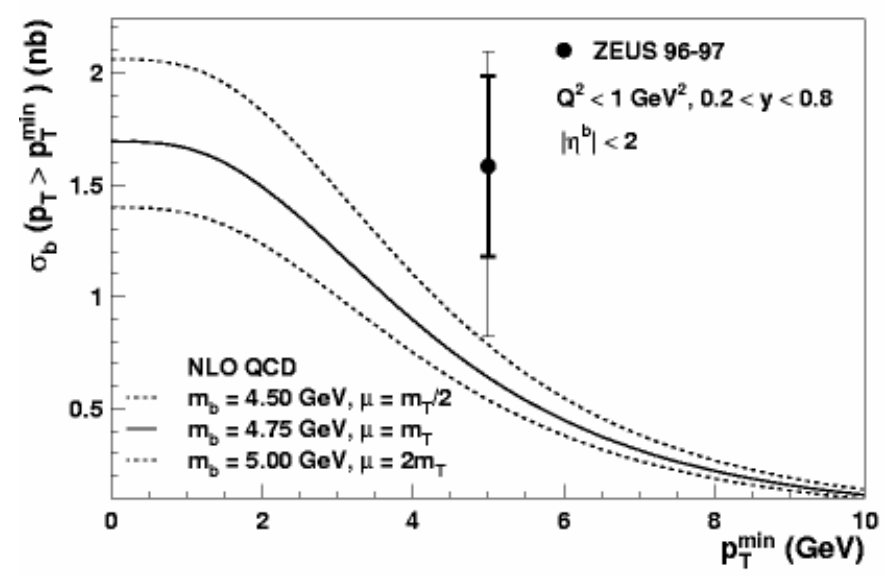

Figure 3: The extrapolated $b$ cross section for a given minimum transverse momentum cut compared with theoretical predictions plotted as function of the transverse momentum cut. The different curves show the predictions for varying $b$-quark masses and a varying factorization and hotoproduction scale.

iv) Study of the Effective Transverse Momentum of Partons in the Proton Using Prompt Photons in Photoproduction at HERA

The photoproduction of prompt photons, together with an accompanying jet, has been measured. A study of the effective transverse momentum, $\left\langle k_{T}>\right.$, of partons in the proton, as modeled within the framework of the PYTHIA Monte Carlo, gives a value $<k_{T}>=1.69 \pm 0.18$ ${ }_{-0.20}^{+0.18} \mathrm{GeV}$ for the $\gamma \mathrm{P}$ center-of-mass energy range $134<W<251 \mathrm{GeV}$. This result is in agreement with the previously observed trend in hadron-hadron scattering for $\left\langle k_{T}\right\rangle$ to rise with interaction energy, see Figure 4.

v) Measurement of Dijet Cross Sections for Events with a Leading Neutron in Photoproduction at HERA

Differential cross sections for dijet photoproduction in association with a leading neutron using the reaction $e^{+} p \rightarrow e^{+}+n+$ dijets $+X_{r}$ have been measured. The fraction of dijet events with a leading neutron in the final state was studied as a function of the jet kinematic variables. The cross sections were measured for jet transverse energies $E_{\mathrm{T}}{ }^{\text {jet }}>6$ $\mathrm{GeV}$, neutron energy $E_{n}>400 \mathrm{GeV}$, and neutron production angle $\theta_{\mathrm{n}}<0.8 \mathrm{mrad}$. The data are broadly consistent with factorization of the lepton and hadron vertices and with a simple one-pion-exchange model. The differential cross section for neutron-tagged events as a function of $x_{\pi}$,. the fraction of the exchanged pion's momentum participating in the production of the dijet system, is shown in Figure 5. 


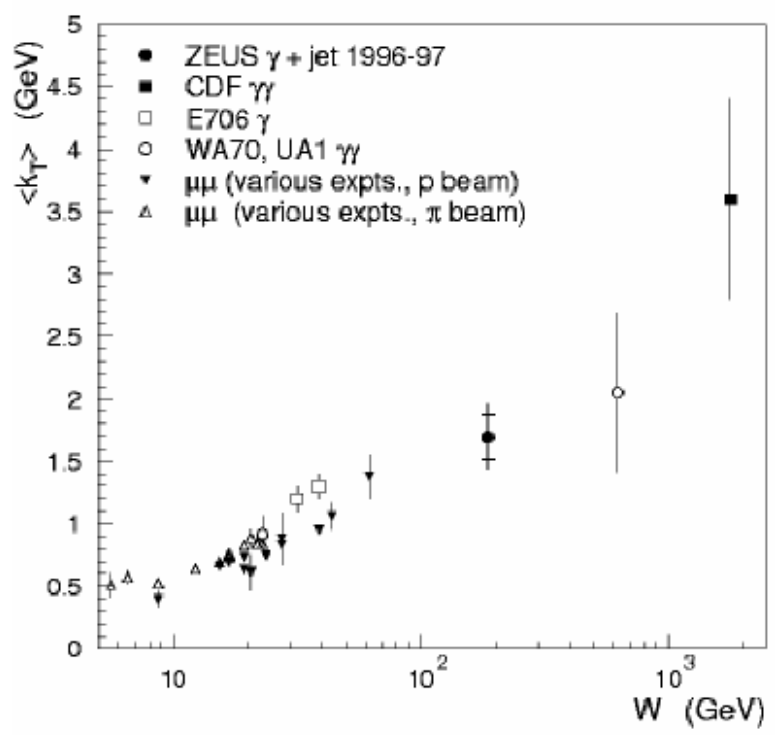

Figure 4: the ZEUS

measurement for $\left\langle k_{T}>\right.$ compared with results from other experiments. The horizontal axis denotes the center-of-mass energy of the colliding particles, which in the case of ZEUS are the photon and proton.

The measured cross section is compared to predictions of the RAPGAP Monte Carlo model using the light-cone pion form factor and the SMRS-P3 pion parton densities. The shape of the $x_{\pi}$ distribution disfavors the monopole form-factor (not shown), but both the shape and normalization are reproduced by the Monte Carlo. The results for other parameterizations of the pion parton densities, which are determined from hadron-hadron interactions, are indistinguishable.

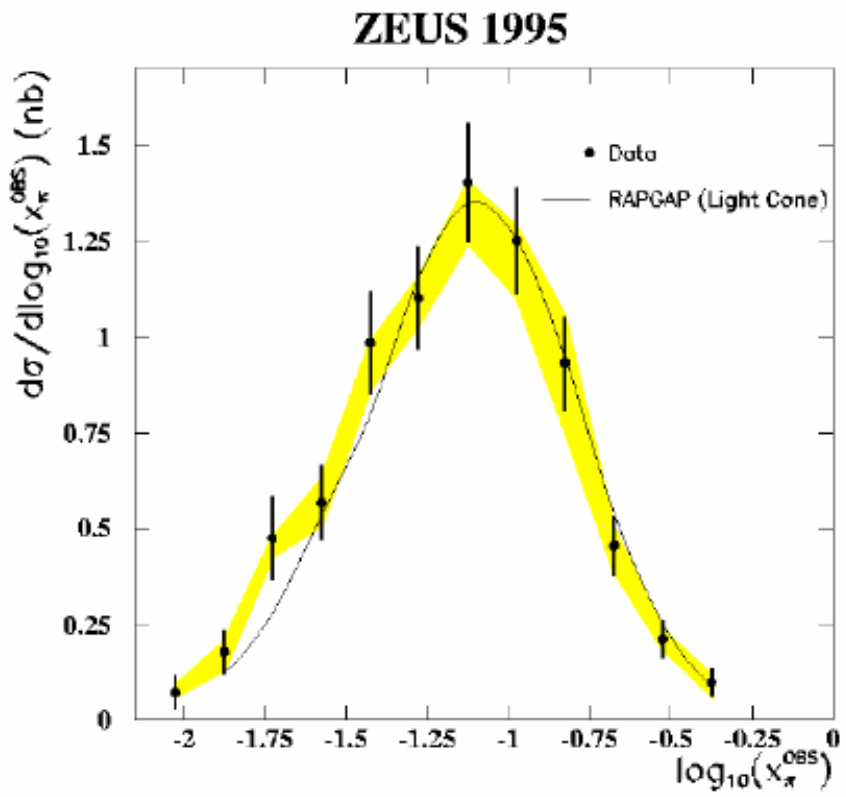

Figure 5: Differential cross section as a function of $x_{\pi}$, the fraction of the exchanged pion's momentum participating in the production of the dijet system for the neutron-tagged sample. The results are compared to the predictions of the RAPGAP Monte Carlo Model using the light-cone pion form factor and the SMRS-P3 pion parton densities. 


\section{b) HERA and ZEUS Operations}

Following the highly successful positron run of 1999/2000 the machine is being prepared for the high luminosity operation. Strong focusing elements (quadrupoles) are being installed close to the interaction points in order to reduce the lateral beam dimensions. The new superconducting quadrupoles have been delivered to DESY and are being tested and measured.

The shutdown period gives ZEUS the opportunity to install new detector components. The new detector configuration will boast a significantly improved tracking system. Precise vertex information will be provided by a Microvertex Silicon Tracker consisting of barrels of silicon detectors in the central region and wheels in the forward region. The tracking in the forward region will be greatly improved by the addition of a Straw Tube Tracker replacing the previously used transition radiation detectors. The next data taking period is expected to commence later this year.

(J. Repond)

\section{I.B EXPERIMENTS IN PLANNING OR CONSTRUCTION}

\section{B.1 Star Detector for RHIC}

The spin physics group is primarily interested in the study of the quark and gluon contributions to the nucleon spin through polarization measurements. Several ANL physicists are members of the collaboration which is building the endcap electromagnetic calorimeter (EEMC) for the Solenoidal Tracker At RHIC (STAR). This collaboration, led by physicists at the Indiana University Cyclotron Facility (IUCF), is building the EEMC to study polarized proton collisions at STAR. From these collisions, information can be extracted about the gluon component of the nucleon spin. A crucial aspect of these measurements is the separation of gamma rays emitted directly from the proton-proton collision from the pairs of gamma rays produced in the decay of neutral pions. This requires a highly segmented shower-maximum detector (SMD) to distinguish the electromagnetic shower of a single photon from that of pairs of photons. Argonne physicists are responsible for the production of this SMD, which will be composed of triangular scintillator strips. The design phase of the SMD module construction was nearly completed during the first half of 2001, and analysis work on cosmic ray data from prototype modules began.

During the first half of 2001, the gluing jig for the SMD modules was received from IUCF, assembled, and tested with the "mechanical model" strips. These extruded plastic strips had the same dimensions as the "good strips," but contained imperfections that made them unsuitable for installation in STAR. Several test gluings were performed in order to 
gain experience and to measure the strip spacing accuracy. The average spacing of adjacent strips was found to be $5.08 \pm 0.24 \mathrm{~mm}$ and the spacing of adjacent pairs of strips (those constrained by the jig during gluing) was $10.17 \pm 0.18 \mathrm{~mm}$. The measurement of the spacing was found to be reproducible within $\pm 0.05-0.07 \mathrm{~mm}$.

A full size mechanical prototype SMD module (subtending 30 degrees) was glued, taken to IUCF, and machined to the proper dimensions. A number of minor changes were suggested after the machining. For example, alignment pins were recommended to locate the glued module on the router bed. Jigs were designed and machined at ANL for this purpose. Small revisions to the module dimensions and to the location of the optical fiber connectors were made. During the mechanical prototype module machining, the average thickness over the surface was measured to be $0.914 \pm 0.022 \mathrm{~cm}$ with vacuum and $0.927 \pm 0.023 \mathrm{~cm}$ without vacuum pulling the module to the router bed.

The suggested changes were incorporated into the operating prototype module, which was glued and taken to IUCF partway through this period. Two methods were tried to install the bushings for the alignment pins, and one was adopted for future assembly. This module was machined and returned to Argonne. The machined ends of the strips were painted with white reflective paint, and wavelength shifter (WLS) optical fibers were inserted into holes in the strips. A few quite minor mechanical problems were discovered, and they will be fixed in the next modules. In general the machined parts, including the glued strips, the fiber routing layer, the aluminum cover, and the metal bumpers, all fit well and work as planned.

The prototype module was then moved to the cosmic ray test facility. The goal was to map the response of the SMD modules strip by strip, as a function of position along the strips. Most of the hardware was complete. However, only about half the planned number of multi-anode photomultiplier tubes (MAPMTs), clear fibers, and cables were installed and working for these tests. The remainder will be put in the setup in the next period. Cosmic ray data were collected successfully for many days.

Several different techniques were used to estimate the light yield from the strips. Software was developed to compensate for the time variations in the ADC pedestals, and a corrected spectrum was obtained for each strip. To eliminate events where the cosmic ray passed through only a corner of the strip, cuts were added to eliminate events with a nonzero signal in either of the neighboring strips. The single-photoelectron (p.e.) peak was clearly seen in the resulting ADC spectra, and for each strip this peak was fit with a Gaussian. Above this peak, the spectra were fit to a function obtained by assuming a Poisson distribution of photoelectrons. The average photoelectron yield for the cosmic ray events was obtained from this fit. The results suggest an average of roughly 4 p.e. or more for a minimum ionizing particle traversing the $6 \mathrm{~mm}$ of scintillator from the peak of the triangle to its base. "This is about double the light yield needed to distinguish neutral pion decays from isolated direct photons." 
A second analysis technique used a small test module of scintillator strips located on top of the prototype SMD module in the cosmic ray test facility. The modules were aligned with the strips being parallel, such that the tips of downward-pointing triangular strips in the test module met the tips of upward-pointing strips in the prototype. More restrictive cuts on the ADC spectra for the prototype strips are permitted in this situation. For a given strip in the prototype, a signal was required in the test module strip immediately above, and in both planes the two neighboring strips were required to have no signal. The derived photoelectron yield agreed reasonably well with the results of the method described earlier.

The calibration of the optical components of the cosmic ray test facility began during this period. WLS fibers from one 8-fiber connector and one 12-fiber connector were simultaneously illuminated by an LED. These mate with the connectors for the clear fiber bundles which are attached to the MAPMTs. Each of the clear fiber bundles was tested to calibrate the combination of the clear fiber efficiency and the MAPMT gain. The relative gains of the 20 WLS fibers were measured using a single clear fiber, which was attached to each of the WLS fibers, one at a time. Studies of the single-photoelectron response of the MAPMTs, made possible by reducing the LED output to a very low level, began near the end of this period.

The light yields of a series of individual scintillator strips were obtained. Approximately 43 strips, selected at regular time intervals during the extrusion process, were measured. The strips were mapped by passing them through a 1-2 $\mathrm{mm}$ wide (FWHM) beam of momentum-analyzed $\sim 2-3 \mathrm{MeV}$ electrons from a ${ }^{106} \mathrm{Ru}$ radioactive source. The light yield as a function of the beam position on the scintillator matched the triangular shape of the strips. Among all the strips, the variation in light output was within $\pm 10 \%$. Ground-up BC408 scintillator from Bicron had been extruded, and four of these strips were also mapped. The average light yield of the Bicron strips was about $10 \%$ higher than that of the polystyrene strips, which is not a statistically significant difference. Tests are continuing.

At the end of the six month period, another SMD module was glued. It is not expected that the upcoming "production readiness review" will lead to changes in the gluing procedure, although it may affect the machining of the module. It is hoped that this module will become the first production module, and that module production on a regular basis will begin soon after the review. 


\section{I.B.2 MINOS - Main Injector Neutrino Oscillation Search}

The MINOS experiment will study neutrino oscillations with significantly greater sensitivity than has been achieved by previous experiments. The phenomenon of neutrino oscillations allows the three flavors of neutrinos to mix as they propagate through space or matter. The MINOS experiment is optimized to explore the region of neutrino oscillation parameter space (values of the $\Delta m^{2}$ and $\sin ^{2}(2 \theta)$ parameters) suggested by previous investigations of atmospheric neutrinos: the Kamiokande, IMB, Super-Kamiokande and Soudan 2 experiments. The study of oscillations in this region with an accelerator-produced neutrino beam requires measurements of the beam after a very long flight path. This in turn requires an intense neutrino beam (produced for the MINOS experiment by the Fermilab Main Injector accelerator) and massive detectors. The rates and characteristics of neutrino interactions are compared in a "near" detector, close to the source of neutrinos at Fermilab, and a "far" detector, $735 \mathrm{~km}$ away in the underground laboratory at Soudan, Minnesota. The neutrino beam and MINOS detectors are being constructed as part of the NuMI (Neutrinos at the Main Injector) Project at Fermilab.

The MINOS detectors are steel-scintillator sandwich calorimeters, with toroidally magnetized 1-inch thick steel planes. The combination of alternating active detector planes and magnetized steel absorber planes has been used in a number of previous neutrino experiments. The MINOS innovation is to use extruded plastic scintillator with fine transverse granularity (4-cm wide strips) to provide both calorimetry (energy deposition) and tracking (topology) information. The 5,400 metric ton MINOS far detector is also much more massive than previous experiments. Recent advances in extruded scintillator technology and in pixilated photomultipliers have made such a detector feasible and affordable for the first time.

Results from Super-Kamiokande, Soudan 2 and MACRO experiments provide increasing evidence that neutrino oscillations are taking place within the region of parameter space that MINOS was designed to explore. Indications from Super-Kamiokande data that the value of $\Delta m^{2}$ is around $3.5 \times 10^{-3} \mathrm{eV}^{2}$ have led to the design of a lower energy beam for MINOS than was initially planned, in order to improve sensitivity at low $\Delta m^{2}$. Argonne physicists and engineers have been involved in several aspects of the preparations for MINOS: scintillator-module factory engineering, near-detector scintillator-module fabrication, near-detector front-end electronics, far-detector installation and the use of the Soudan 2 detector with the NuMI neutrino beam (also known as Theseus).

One major focus of work by the Argonne MINOS group is scintillator module construction. (MINOS detector modules are subassemblies of 20 or 28 extruded plastic scintillator strips.) During the first two months of 2001 the group completed the assembly and testing of prototype near detector modules in the new fabrication facility in Building 369 
at Argonne. The group began routine module production in March and, by the end of June, had completed 108 near detector modules (out of the total 573 required). Scintillator module assembly is now taking place at "factories" located at Caltech, at the University of Minnesota in Minneapolis and at Argonne (where all near detector modules will be constructed). Argonne physicists and engineers serve as NuMI Project Level 3 WBS Managers for the design and construction of the machines needed to construct scintillator modules and for the operation of the three module factories. Previously, an Argonne physicist served as the Level 3 manager for scintillator strip fabrication and was responsible for the introduction of several important strip design innovations.

The Argonne MINOS group constructed and operated two prototype production facilities for far detector scintillator modules in Building 366 during 1999 and 2000. The prototype modules were used to evaluate production procedures and to instrument the 4-plane prototype setup at Fermilab. The 4-plane prototype was a full-size assembly of MINOS fardetector steel and scintillator planes that was operated during 2000. Modules built during the May 2000 prototype production run met all performance criteria and allowed the fabrication of module assembly equipment for the Caltech and Minneapolis factories to begin at Argonne.

Argonne built the assembly machines and tooling for the Caltech, Minneapolis and Argonne module factories. The Argonne mechanical support group also fabricated and installed the scintillator module mapper at Soudan during the first half of 2001. Figure 1 shows the Soudan mapper in operation in the underground laboratory. By the end of June the Soudan mapper was in routine operation and all three factories had achieved or exceeded the planned module assembly rates in routine production. The setup of the near detector module factory in Building 369 at Argonne was completed in early 2001, in parallel with production of the first prototype near detector modules. The preparation of Building 369, which has air conditioning and more floor space than Building 366, was made possible by substantial financial and logistical assistance from the Laboratory administration. 


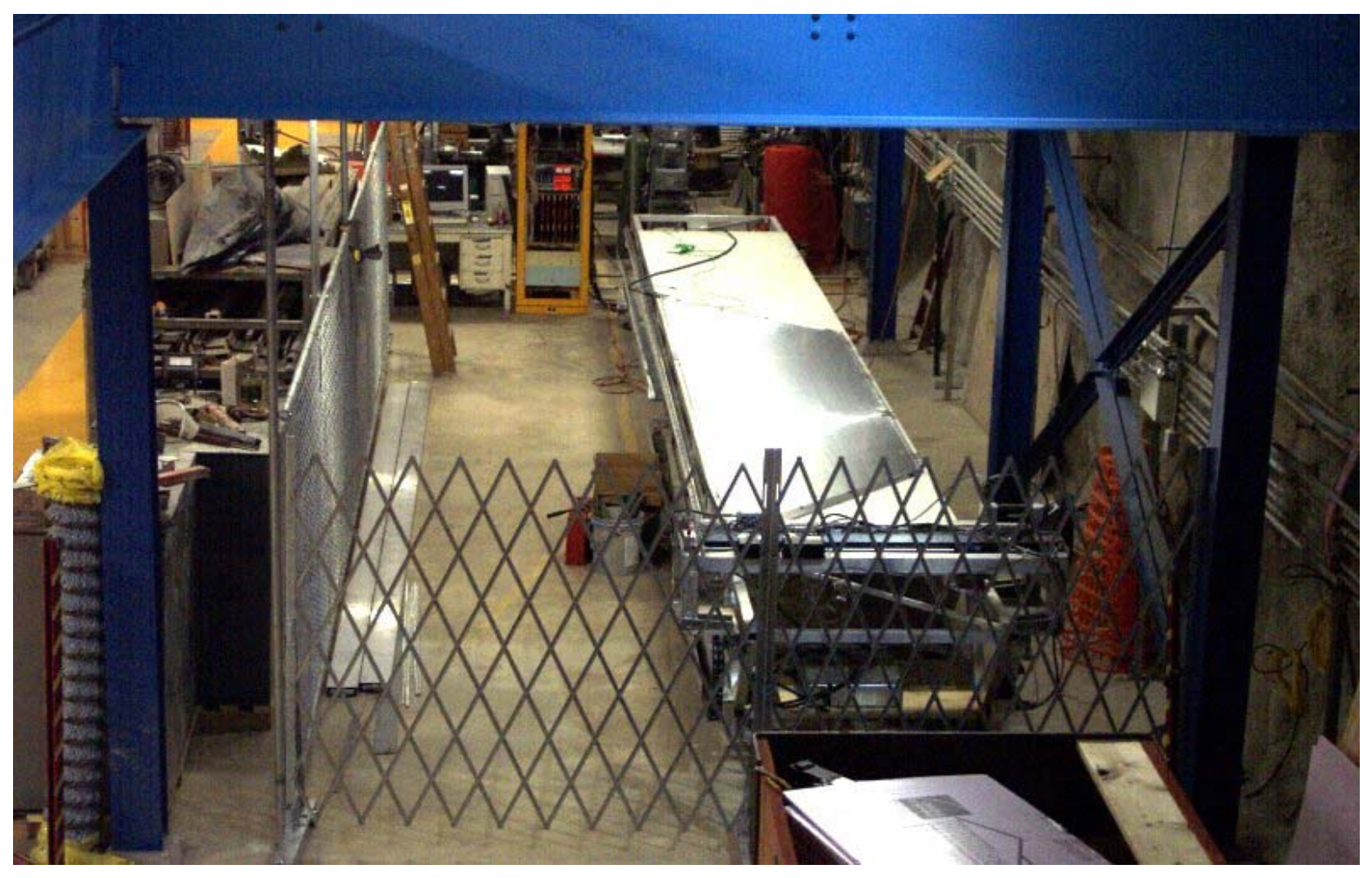

Figure 1: Photograph of the Soudan module mapper in operation in the underground laboratory at Soudan. The mapper uses a radioactive source and a Rabbit data acquisition system to make a detailed map of scintillator module response. Mappers at the Caltech and Minneapolis factories measure each module before shipping to Soudan. The Soudan mapper is used to check for changes in response during shipping and rigging down the Soudan mineshaft. Only a few percent of modules mapped at Soudan have shown changes in response. Mappers at the three factories and at Soudan were designed, fabricated and installed by the Argonne HEP Division physicists and engineers.

The second major focus of the Argonne MINOS group is electronics and data acquisition for the experiment. Argonne physicists and engineers serve as the Level 2 manager for electronics and the Level 3 manager for the near-detector front-end electronics. Figure 2 shows a block diagram showing the relationship of the near-detector front-end electronics boards. The 1999 decision to use single turn extraction (STE) from the Main Injector for the NuMI neutrino beam required a change to the current design of the neardetector front-end electronics, which is based on the Fermilab QIE ASIC chip. Prototype QIE chips produced in 2000 were evaluated by Fermilab and Argonne engineers and were found to meet or exceed all performance requirements. The chips operate at $53 \mathrm{MHz}$ and have no digitization deadtime, which is essential for the operation at the high instantaneous rates produced by STE. 


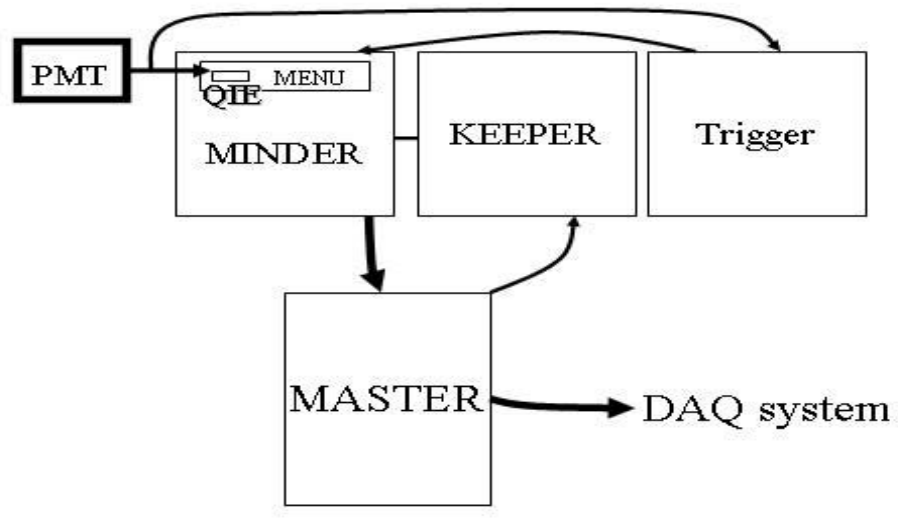

Figure 2: Block diagram of the near-detector front-end electronics. The Argonne electronics group is responsible for the design and fabrication of the MINDER, KEEPER/Trigger and the MASTER module. Fermilab is providing the QIE chip and the MENU module. MINOS UK collaborators are providing the Hamamatsu M64 PMTs and the DAQ system.

During the first half of 2001 Argonne engineers completed the fabrication and testing of the first prototype MINOS MASTER module, which receives and processes the data from the QIE chips. In order to test this complex VME board, a test stand was assembled in 2000 using a prototype version of the MINOS 9U VME crate. Software was written to interface to the MASTER module. This made use of the VX-Works operating system, which will also be used for the final MINOS data acquisition software, to simplify the later porting of the test code to the DAQ. The MASTER module was extensively tested using its internal diagnostic memory to simulate real data from the QIE chips.

The Argonne group's work on the final layouts of the remaining near-detector frontend circuits (the KEEPER/Trigger modules, the MINDER cards, and the MINDER crate) progressed well during the first half of 2001. The protocols for communication among the various boards are being developed along with the board designs. By the end of June the Argonne electronics group was working closely with engineers and physicists from Fermilab and IIT to complete the layout and fabrication of the MENU module (which holds the QIE) and the VTM and MTM timing boards. These will be needed for the small system test scheduled for September. The Argonne group also began work on software to operate and study the performance of the readout electronics chain in the small system test. The group also developed simulations of electronics response to evaluate the physics impact of the choices made in the final stages of board design. 
An Argonne physicist also serves as Level 2 manager for far detector installation. Installation planning during the first half of 2001 involved close interaction with MINOS collaborators who are building detector and electronics components that will be installed at Soudan during the summer of 2001. Excavation of the MINOS far-detector cavern at Soudan began in May 1999 and was completed in December 2000. The construction of the MINOS surface receiving building was also completed in December. Outfitting of the surface building, which is used to receive and process detector steel plates and scintillator modules, was completed during the first half of 2001. The contractor for installation of the underground cavern infrastructure began work at the end of 2000 and made excellent progress during the first half of 2001. This work was on schedule for completion in July. Beneficial occupancy of the underground cavern at that time will be followed by the installation of the 5,400-ton far detector. By the end of June the Soudan laboratory technical staff had moved first steel plates and first modules crates into the underground laboratory in preparation for the assembly of the first detector planes later in the summer.

(D. S. Ayres)

\section{I.B.3 ATLAS Detector Research \& Development}

\section{a) Overview of ANL ATLAS Tile Calorimeter Activities}

The TileCal subsystem continued making excellent progress in the first half of 2001. Both submodule and module mechanical construction are proceeding on schedule (Fig. 1) with 163 submodules and 36 modules constructed. In addition, the module instrumentation rate has achieved our original goal of 1 module per month with 16 modules having been instrumented at Argonne and tested. The shipping of modules to Michigan State University and to CERN continues to be routine. Twenty-six completed modules have now been shipped to CERN. In addition, we now routinely run the cesium source in all modules instrumented at MSU and replace tiles and repair fibers as needed. As is shown below for all recent modules, we typically have no tile-fiber couplings outside the design goal of having a response less than $75 \%$ of the average. 


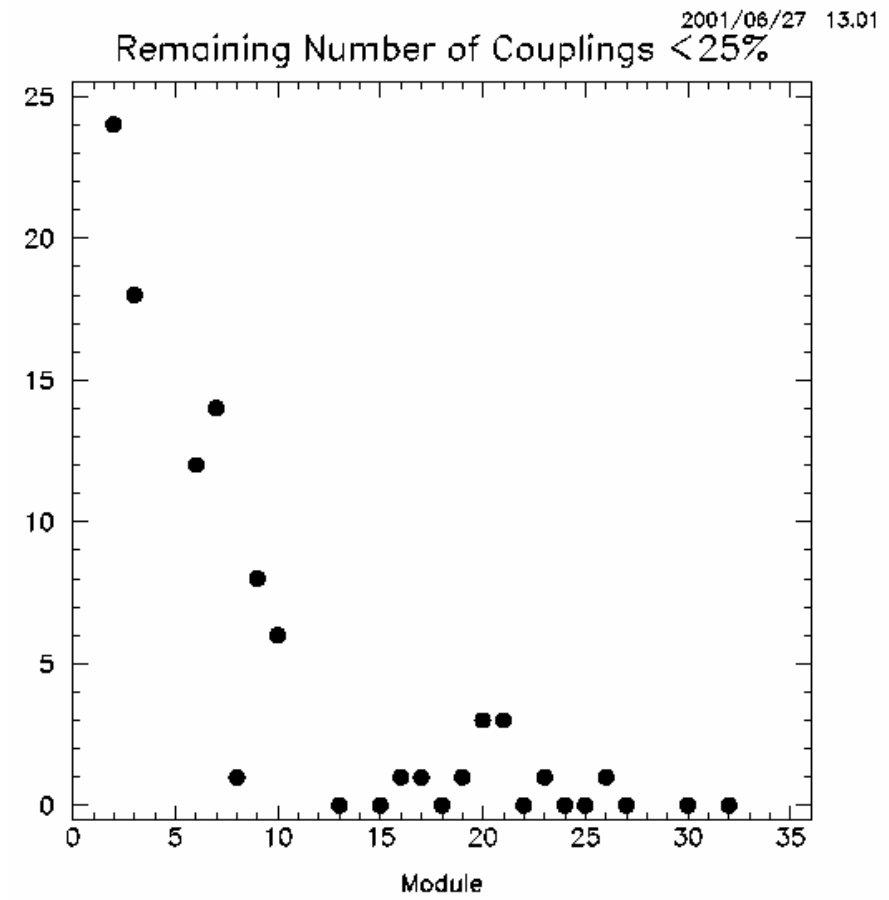

Figure 1: Number of couplings failing design goal by module number.

(J. Proudfoot)

\section{C. DETECTOR DEVELOPMENT}

\section{I.C.1 Atlas Calorimeter Design and Construction}

The ATLAS Tile Calorimeter construction effort is now fully into the production phase. The areas of ongoing work comprise: submodule construction; module assembly; instrumentation and testing; testbeam measurement of detector performance; engineering support of work at US collaborating institutes; continued engineering evaluation of specific elements of the detector and final design of areas in the detector where special constraints, such as the support of the liquid argon cryostats, must be accommodated.

\section{a) Submodule Construction}

Submodule production continued at the scheduled production rate. At the close of this reporting period, 163 submodules were stacked and welded, 126 painted and 126 fully completed and stored for mounting into modules. The height envelope for all submodules constructed to date at Argonne is generally well within the tolerance envelope. We expect to complete production of our assigned quota of 192 submodules by late fall 2001. The repair and replacement of defective submodules will likely extend submodule construction activities to the end of 2001. Engineering analysis completed in 2000 indicated that the number of bolts in the mounting bars needed to be increased from 3 to 5 for a number of submodules in 
the region of the support saddle. The fabrication of these bars was carried out in early 2001 and about half of these (slightly) special submodules have now been constructed.

Submodule production has been completed at The University of Chicago, where 194 submodules were stacked and all submodules and remaining materials have been shipped to Argonne. However, some of these submodules are not to specification:

- 6 were shipped unpainted because their height exceeded the design height by as much as $2 \mathrm{~mm}$. These will be repaired by removing a single plate of which its thickness will be reduced before it is re-attached to the submodule, then painted and put into service.

- 6 were only tack-welded since the slots in these modules had been deformed. These will be repaired by grinding off the welds and completely re-stacking the submodule.

- 6 were completed but had a number of deformed slots. These will probably be scrapped and replaced by submodules built from scratch (material to accomplish this is being shipped to Argonne from European collaborators who have completed their production). This work will be carried out at Argonne in the fall of 2001.

The University of Illinois has continued to construct submodules at a faster rate than originally planned. They have now completed 150 out of 192 total to be built and estimate that they will complete production of submodules by the end of calendar year 2001 . No problems have been encountered in this production.

The design of the special submodules required in the region of the endcap cryostat supports is now complete. We have developed a plan and approach to be used for the fabrication of these submodules. A first production special submodule was constructed in early 2001 as proof of principal. We envisage commencing full-scale production of the submodules in October 2001.

\section{b) Module Assembly and Shipping}

Module production is also proceeding smoothly and at a somewhat higher rate than planned in the baseline schedule. A cumulative total of 36 modules have been constructed to date and we routinely construct one module every 2 calendar weeks. Girder production is still ahead of our requirement and the vendor is producing them at the rate of 8 every 3 months. Forty-two have been completed and we expect to complete the fabrication of this component in 2001. Witness inspection of each series of 8 girders has identified only minor problems and we are considering dropping this through the remainder of the procurement and instead relying only on the QC data provided by the vendor. The timely delivery of the special ITC submodules from the University of Texas at Arlington is still a concern, but so 
far we have not been forced to halt production while waiting for the delivery of the next series. The first 2 and simplest of the special ITC submodules have been delivered and we do not expect the production of the remaining special ITC submodules to have any technical difficulties.

The module design envelope is $+/-0.75 \mathrm{~mm}$ (0.029in) and we verify this in two ways: using a plumb-bob prior to welding in the inner radius plate, and using a 10-ft. precision straight edge and feeler gauges to measure the relative non-planarity at many locations on the module surface. An updated plot of the feeler gauge summary data is shown in Fig. 2, which shows that by all measures our modules are meeting the design specification and are uniform on average from module to module.

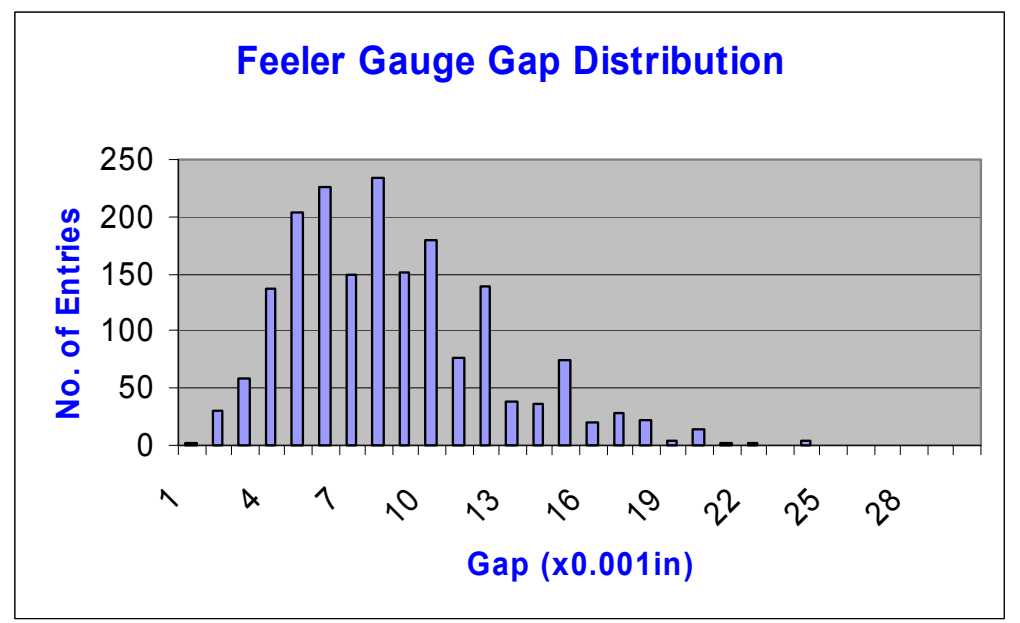

Figure 2: Module envelope deviation measured using a precision straightedge and feeler gauges at many points on the module surface.

\section{c) Module Shipping}

Shipping of modules to Michigan State University and CERN has continued with no problems. 26 modules have now been sent to CERN and 19 have been shipped to MSU (16 of these have been instrumented and returned to Argonne). We are at present behind our scheduled shipping plan by 2 modules. However, we expect to recover this by the end of the summer.

(J. Proudfoot) 


\section{d) Instrumentation and Testing}

Module instrumentation and testing is now a routine activity and two full crews of technicians are trained in the procedures. At the present time 16 modules have been instrumented at Argonne and 9 at Michigan State University out of a total of 32 to be instrumented at each location. We are meeting our planned schedule and have improved our instrumentation time per module from 35 days to typically less than 28 days. With improved procedures and supervision we have optimized the overlap of tasks that can be carried out concurrently. In addition, we are generally now able to buffer the instrumentation activities by having an extra module ready for preparatory work while one module is undergoing cesium testing. This buffer was used also to accommodate the MSU group who wished to increase their rate of module instrumentation through the summer months when student help was more available.

We continue to closely monitor the variation in light output with tile pack. Our sorting procedures are working and we continue to get an average layer uniformity which is of order $6 \%$ (c.f. the specification of $<10 \%$ ). Our own work is best represented by looking at instrumentation uniformity, determined using variables which remove systematic affects from the scintillator tiles. This is shown in Fig. 3. The observed non-uniformity of $4-5 \%$ is commensurate with the tile-to-tile variation of $\sim 5 \%$ and fiber-to-fiber variation of $\sim 3 \%$.

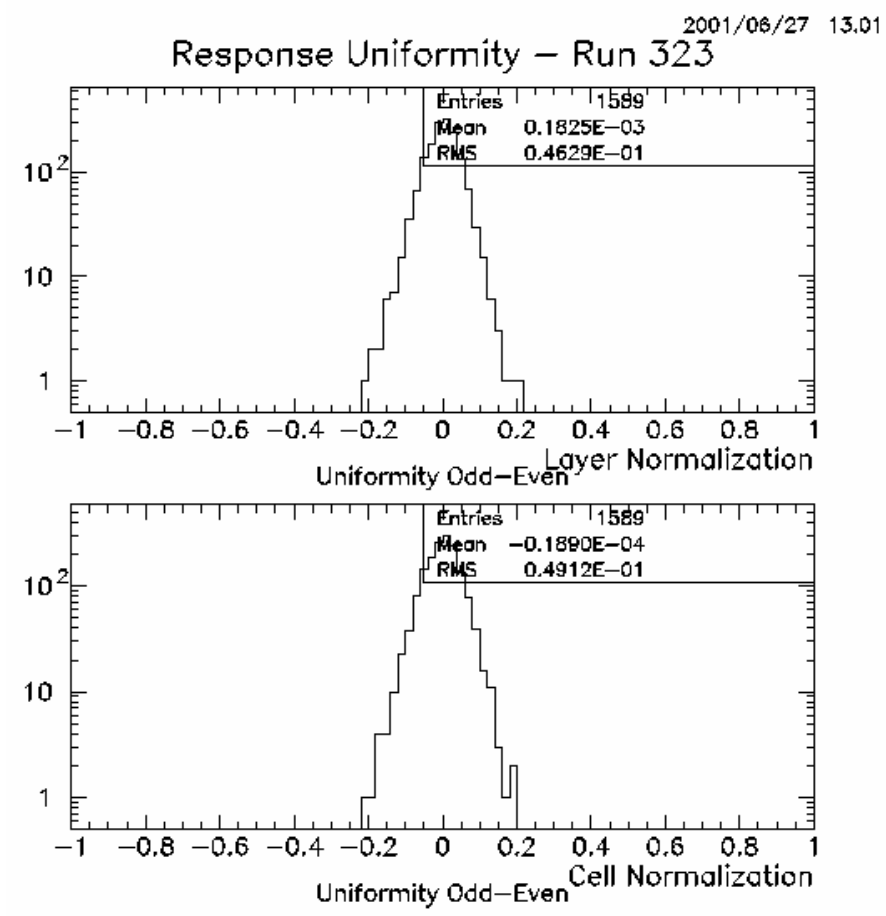

Figure 3: Instrumentation non-uniformity determined by comparing left and right tile-fiber couplings by scintillator tile. 
A second measure of the quality of the instrumentation work is the number of tilefiber couplings in a completed module, which has a response less than $75 \%$ of the average. This is shown above in Fig. 1. In general, at Argonne we now achieve $100 \%$ of couplings passing this selection. In most instances, this has not been the case in the modules instrumented at MSU and, in several instances, we have carried out repairs and reached this level by replacing fibers and/or tiles. In a few exceptional cases, these repairs have not been possible and the fibers in question will be replaced once the modules are delivered to CERN using a special technique developed there and which has not been replicated in the U.S.

In 2000, we set aside one module for repeated measurements using the cesium source as a test of the overall stability of the optical system, without the uncertainty of changes induced by shipping and handling. A first re-measurement showed little change in response or uniformity and the study will continue until the end of 2001. Finally, R. Stanek, in collaboration with a summer student, has started incorporating all of the quality control data from both mechanics and optical performance measurements into a relational database.

(J. Proudfoot and R. Stanek)

\section{e) Test Beam Program}

An Argonne staff physicist continues to be the coordinator of the Tile Cal testbeam program. In addition, a Division computer scientist continued to develop readout code for the online data acquisition system. Three testbeam periods are allocated to the Tile Calorimeter in July, August, and September. This period was devoted to developing a calibration program, ascertaining that the drawer electronics will be reliable and functional, and verifying timing of the trigger cables.

The DAQ will be set up in a similar configuration as last year, with 2 RIOs for RODs with 3 G-link inputs on each, a beam crate, an Event Builder Crate with a $100 \mathrm{Mb}$ link to a PC, and a TTCpr in each of the 3 crates. The drawer electronics will use a Chicago interface board having simplex G-link outputs. The LDC receivers are CERN ODIN boards that piggyback on a PMC card. The new problem HEP had to solve was to accommodate three of these cards in the CES PMC extender module. Homemade, high-speed connectors were designed and constructed. These were debugged at Argonne before shipment to the testbeam.

A meeting was called by HEP staff at CERN to discuss the calibration issues, because this year starts the beam certification program. One important issue is the gain setting of the phototubes. In previous years, the gain was set to $0.9 \mathrm{pC} / \mathrm{GeV}$. As the light 
yield in the calorimeters increased over the years, we were finding gains that approached $1.1 \mathrm{pC} / \mathrm{GeV}$ and, in fact, $\mathrm{Cs}$ scans at Argonne predicted a 30\% larger yield in future modules.
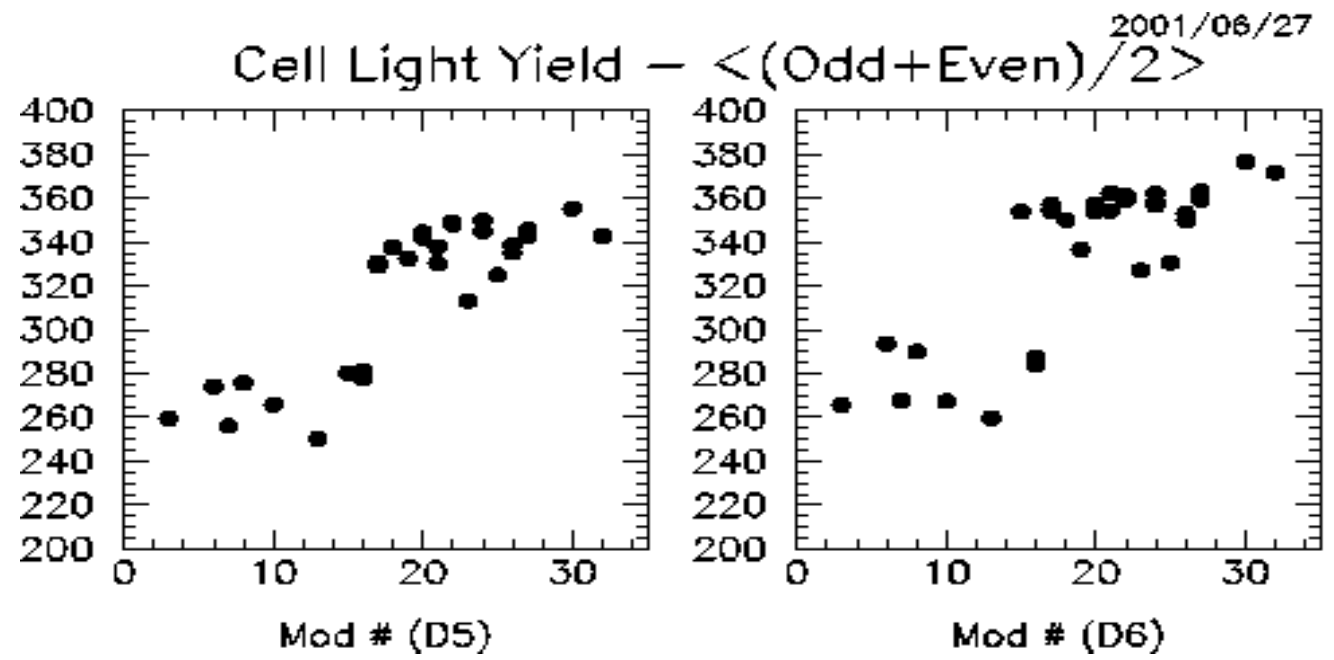

Figure 4: Average cell light yield as a function of module number showing the increase in light yield for later batches of scintillator tiles.

A major problem with the increase in light yield is the electronics saturation at 1.6 $\mathrm{TeV} /$ cell in ATLAS. The HV system was designed to operate at the $0.9 \mathrm{pC} / \mathrm{GeV}$ region, and non-linearity will increase as the HV is lowered. These discussions are ongoing.

In May, a working meeting was held at Clermont-Ferrand to ensure that the production drawers were constructed correctly and the certification procedure was operational. HEP staff participated in this meeting and several problem areas were discovered before final integration. The first problem seen in the assembly was that the ground planes of the electronics were physically connected to the steel structure. The second problem found was that the Chicago interface card produced corrupted data. This latter issue has to be dealt with by the Division computer scientist in July.

Associated with the assembly of the drawers is the issue of the trigger cable lengths within the drawer. We compared the data obtained from pions in the testbeam to that calculated by fiber lengths and the dimensions of the calorimeters. These numbers were used by the trigger group to determine the lengths of cables inside the module. Several people have done these calculations, and agreements are good, as well as agreeing with beam measurements. 


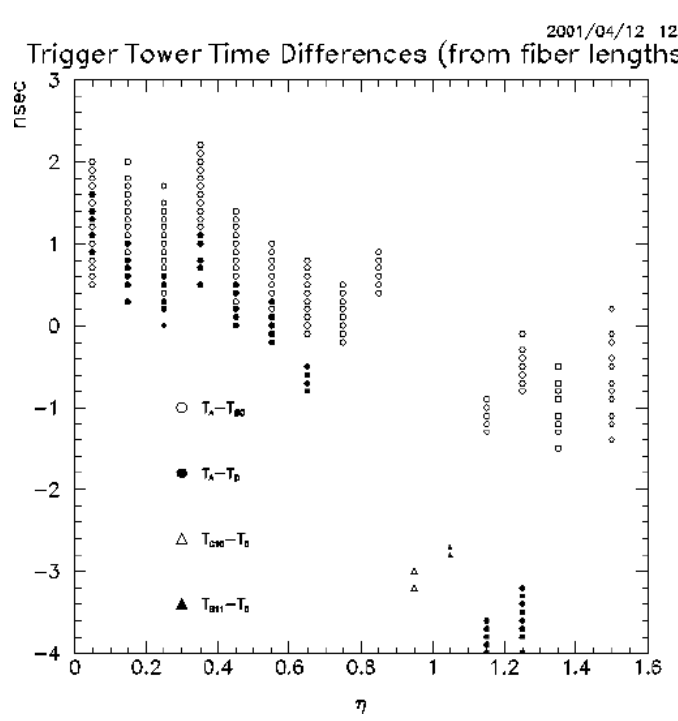

a) Calculated relative timings from the first sample in the calorimeter. The various symbols refer to where in the stack the signal is referenced.

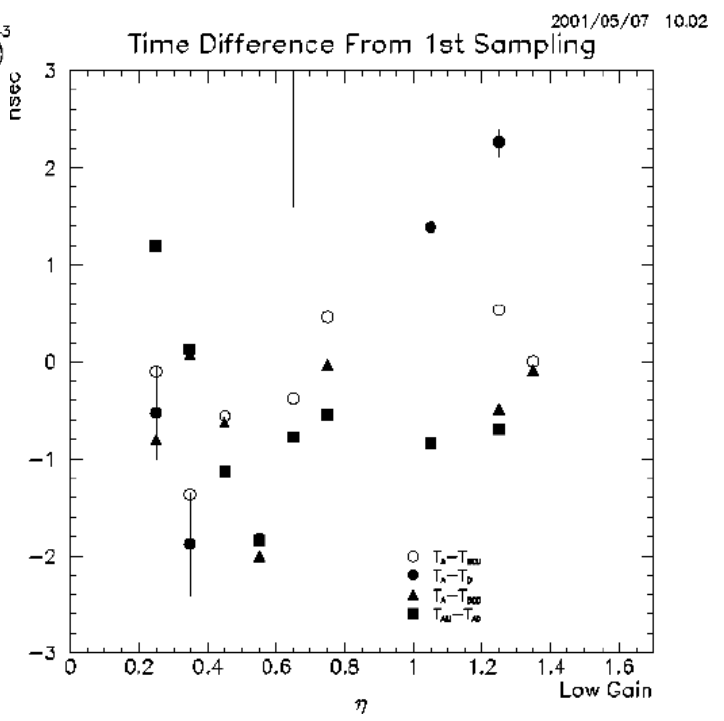

b) Relative timings from the first sample in the calorimeter for pion showers.

Figure 5: Tower signal timing: a) calculated relative to $1^{\text {st }}$ sample; b) for pion showers.

(R. Stanek)

\section{f) Engineering Design and Analysis}

Substantial progress has been made in several areas of the engineering work in which Argonne staff have major responsibilities. Fabrication of one of the special submodules required for the endcap cryostat support was completed and this submodule has been shipped to CERN for use in tests. Argonne has agreed to take responsibility for several of the engineering tasks associated with assembly of the calorimeter as a whole and its support system. These tasks include: engineering analysis in which V. Guarino is responsible for the summary of engineering calculations, finite element analysis of the cylinder and the design of the barrel and extended barrel saddle supports. The other engineers are contributing to assembly tooling, pre-assembly and assembly plan. Essentially, all engineers are involved in the engineering analysis.

Activity in the first half of 2001 focussed on the detailed design and analysis of the endcap calorimeter support saddle. Our initial goal was to modify the solid plate design proposed by collaborators in Europe to one based on fabrication using segmented plates. Analysis, however, showed that such a design would not meet the stress and deflection requirements for the structure and we have followed this design with one implementing a more rigid structure shown below in Fig. 6. Engineering calculations are proceeding well. The deflections are acceptably small and stresses are generally below the design maximum value. A complete documentation of this work is in progress as part of the necessary 
documentation for approval of the structure by Atlas Technical Coordination. This work will be completed by the end of September and it is intended that procurement of one set of saddles will be initiated by the end of 2001 .

Submodule and Module Support Activities: Argonne's scientific and technical staff has continued their support of the supported, submodule construction activities at the other US collaborating institutions. This has included:

- ITC construction and quality control

- Site checks of submodules at The University of Chicago

- Submodule quality control monitoring for the Tile Calorimeter subsystem as a whole.

(J. Proudfoot and V. Guarino)

\section{I.C.2 Computational Projects}

\section{a) ATLAS Computing}

Argonne has continued in its role as joint leader of the ATLAS database development effort. This effort includes not only a multi-petabyte event store (2PB/year is our present best estimate), but also databases for time-varying information such as calibrations, run conditions, slow control information, and alignment. The database domain is responsible as well for most detector description software, and for substantial components of the ATLAS event model.

A major database accomplishment in the first quarter was the introduction in ATLAS software release 1.3.0 of database support for Athena, the ATLAS control framework. The principal component of this support is what the Gaudi/Athena framework calls a conversion service, which handles mappings between transient data (as seen by physics programs) and persistent representations (as stored in databases). A conversion service that supports the ATLAS baseline database technology, Objectivity/DB, was introduced by the ANL database group. Standard Athena tutorial examples, used to illustrate how an algorithm might write data for reading by downstream algorithms, were demonstrated to run successfully, unmodified, with the data stored in and subsequently retrieved from an object database. Persistence capabilities were provided in support of collections of events in HepMC format, the common format used by ATLAS and other experiments to represent the output of physics generators such as ISAJET and PYTHIA. Persistence for the Athena-based version of Atlfast, the principal ATLAS fast simulation program, was also demonstrated. At the request of the Architecture Review Committee, the ability of the core software to maintain independence of database supplier was also demonstrated. 
The freeware relational database MySQL has been employed in the liquid argon community for certain run bookkeeping and storage of testbeam calibration data. BNL has developed database software in support of these applications, and has done so in a way intended to make the software viable as a generalized service accessible through the Athena framework. This work is being integrated into the overall database effort, and ANL has begun support of MySQL as a recognized external package for ATLAS.

Significant time was spent on interaction with the Architecture Review Committee, whose final meeting prior to writing the closeout report was held on 5-7 March. The committee took particular interest in plans for database technology evaluation, which will largely be done in a 2002 timescale.

The bulk of ANL database development effort in the second quarter reporting was directed toward support of a successful major release of ATLAS software, Release 2.0.0--the so-called "Lund Release" (named for the physics workshop held in Lund that utilized this release). Two major components of this work are the database infrastructure needed to support the ATLAS fast simulation program (Atlfast) in Objectivity and (generic) ROOT, and support for interobject references. At the request of the ATLAS Physics Coordinator, together with the Computing Coordinator, a persistence-capable version of Atlfast became a centerpiece of the "Lund program." This was achieved, despite the need to respond to extremely rapid changes to the objects that would have to be made persistent.

Now that the Lund release has entered bug-fixing stages, we hope to effect a transition of responsibility for Atlfast persistence to the Atlfast package developers themselves. In the coming months though, it will be necessary to provide whatever support is needed to allow the physics agenda to succeed.

A joint event model/database workshop was held at Argonne in early April to ensure synchronization of StoreGate (the mechanism by which event data is accessed) and database plans. We expect the work on SmartRef persistence to transfer well to the StoreGate DataLink/DataBucket model. Database support for StoreGate DataLinks and DataBuckets is a principal deliverable expected in the next major release of ATLAS software.

A substantial amount of U.S. database effort in the second quarter has been directed toward laying the groundwork for development of an ATLAS database architecture document. This has, to date, been largely an Argonne/University of Chicago activity, and the time has come to extend this to include CERN and other international collaborators. Several U.S. core database developers will spend the week of 16 July at CERN to accomplish this collaboration. A draft architecture document is intended to be ready for promulgation by late summer. 
In grid-related database work, a Globus replica catalog running at Argonne, with catalog query and update clients running at Brookhaven, has been successfully demonstrated and exercised, both from a command line interface and from $\mathrm{C}$ programs. This is a first step in grid-enabling data access from Athena.

Two abstracts written and submitted by ANL core database developers and their international collaborators have been accepted in this quarter for the Computing in High Energy and Nuclear Physics Conference 2001 in Beijing (CHEP'01): Chisholm et al., The ATLAS Data Management Architecture; and Malon et al., Grid-Enabled Data Access in the ATLAS Athena Framework.

Major deliverables in the next half are expected to include the database architecture document mentioned above, database planning for Data Challenges 0 and 1, and database support for the new StoreGate backend. Conversion service support for input to the detector data service, and a collection registration prototype, are also due, as are a number of pieces of database infrastructure that are primarily the responsibility of Orsay, but that will require some corresponding U.S. effort. Migration to Objectivity 6.x, which will require significant changes to the ATLAS build infrastructure, will also occur in the third quarter. One of the major uncertainties in core database planning in the near term is the required support for pileup, since the scope of the core database component of this effort depends strongly on the (transient) computing model for handling pileup events.

(T. LeCompte)

\section{I.C.3 MINOS Detector Development}

During the first half of 2001 the Argonne MINOS scintillator group completed the transition from detector development to production fabrication. The Argonne mechanical support group built and installed most of the equipment for the Caltech, University of Minnesota and Argonne module factories and participated in the commissioning of both factories. By the end of June the setup of the near detector module assembly facility in Building 369 was complete and all three factories were routinely producing modules of high quality at or above the planned full rate.

During the first half of 2001 the Argonne electronics group completed the specification, design and most of the layouts of the near-detector front-end modules for which it is responsible. The first test of prototypes of all these components operating together, the small system test, is scheduled for September.

The Argonne electronics group also continued to provide support for a number of Rabbit electronics readout systems for MINOS scintillator test setups at Argonne, Caltech, Fermilab, Minnesota, Oxford, Soudan, the University of Texas and the University of Athens 
in Greece. These systems make use of existing Rabbit electronics cards and crates, which became available after the CDF upgrade at Fermilab. MINOS uses these systems for the module mapper readout, photomultiplier testing and for data acquisition from the 4-plane prototype. Several MINOS Rabbit systems have been brought into operation and are now in routine use.

(D. S. Ayres)

\section{I.C.4. Electronics Support Group}

CDF: Work continued in the development and production of front end electronics for the Shower Max Detector of the CDF Upgrade at Fermilab. For this project, we have major responsibilities for the electronics engineering of the system. The system has 20,000 channels of low-noise electronics, and services two detector subsystems. The primary responsibilities involve the coordination of the design engineering and system integration for the entire system, overseeing the production of all components, and ensuring that the overall system meets performance requirements. The development work is a collaborative effort between Argonne and Fermilab.

In this period, we completed the production work for the electronics. All electronics were installed on the detector and commissioned for operation. We were successful in meeting the schedule goals in getting ready for Fermilab Run II.

Argonne was responsible for the design and development of several components of the system. One project was the design, testing, and production of the $\sim 6000$ daughter boards used in the front end electronics, which contain a custom integrated circuit designed at Fermilab called the SMQIEs. The daughter boards are called SQUIDs. Each SQUID contains two SMQIEs, and also other support circuitry for calibration. The SMQIEs digitize charge inputs from the detector continuously, without deadtime. The digitized data is stored locally in a pipeline inside the chip pending a trigger decision. The data is read from the chip when there is a trigger accept. Production of the SQUIDs was begun in September 2000. Argonne was responsible for overseeing the checkout effort, which was done by a crew of 8 technicians at Fermilab. The production was completed in March 2001, with all channels installed and functioning on the detector.

Argonne was also responsible for the design, testing, and production of $\sim 15,000$ preamplifiers, used in the front end electronics of the Shower Max system. These are used on the strip chambers in the Central Barrel of the detector to provide additional signal amplification before the charge signals are sent to the SQUIDs. The preamp provides amplification to the charge pulse from the detector using low-noise, high-speed analog circuitry. The preamps are constructed as small SIPs (Single In-Line Package) using surface

mount parts. The production was begun in December 2000. Technicians at Fermilab 
performed the checkout work. After the preamp SIPs were tested, they were mounted on mother boards. The boards were then tested at Argonne before being installed in the CDF detector. The production was completed in March 2001, with all channels installed and functioning on the detector.

ATLAS: We have major responsibilities in the development of electronics for the Level 2 Trigger of the ATLAS Detector at CERN. Working with colleagues from Michigan State University, we are responsible for the development of two parts of this system: the Level 2 Trigger Supervisor, and the Region of Interest (ROI) Builder.

The ROI Builder is the interface between the first level trigger and the second level trigger. When an event occurs in the detector, signals are sent from the front end electronics to the Level 1 Trigger. The Level 1 Trigger collects event fragments from all over the detector, and stores them in a buffer. It evaluates the data, and identifies regions of the detector that could have an interesting event. The Level 1 Trigger boards then sends a list of addresses called pointers to the ROI Builder, identifying where the event data from the "Region of Interest," can be found. The ROI Builder collects the pointers for the event, and "builds" the event using the pointer list. It then sends the result to the Trigger Supervisor for distribution to Level 2 processors. The ROI Builder is highly complex, using fast, highdensity Field programmable Gate Arrays (FPGAs) to implement the functionality.

In this period, testing continued on the prototype system. We have a working system at CERN, where system tests are being performed. In the early part of 2001, the ROI Builder was used in integration tests for different detector subsystems. The tests were largely successful. We are providing much of the software development and support for this phase of the project.

Another project that we are doing for ATLAS is the development of an interface card for the clock system, called the TTC Mezzanine card. Clock and control information is passed to different parts of the detector on optical fiber in an encoded format. Engineers at CERN have developed a custom integrated circuit called the TTC. The device receives the serial data, and converts it to the appropriate control signals needed by the various subsystems. The card designed at Argonne hosts the device, which is implemented using ball grid array (BGA) technology. The card itself has a PCI format, which is a standard used by digital processors to interconnect auxiliary data streams. In 2000 we designed the first prototype and built 4 cards for evaluation. In this period, we designed a new prototype that uses a new version of the TTC chip. The new chip has additional features that enhance performance. We built four boards, and they are currently being tested and evaluated both at Argonne and at CERN.

MINOS: We continued our work with MINOS, the Neutrino Oscillation Experiment at Fermilab and the Soudan mine. There are two detectors for this experiment. The Far Detector is located in the Soudan Mine in northern Minnesota. The Near Detector is located 
at Fermilab. A beam of neutrinos is created from a beam "spill" of protons at Fermilab. The neutrinos pass through the Near Detector, and are directed through the earth towards the Far Detector. The two detectors are used in conjunction to study how the neutrinos change nature as they pass through the earth. We have major responsibilities for this experiment, including Level 3 Management responsibility for the front end electronics for the Near Detector, as well as responsibility for a large portion of the front end design for that detector.

The heart of the front end electronics for the Near Detector is a custom integrated circuit designed at Fermilab, called the QIE. The QIE digitizes continuously at $53 \mathrm{MHz}$. The operations are pipelined so that there is no deadtime due to digitization. The digitized data will be stored in a local memory during the entire period of the beam spill. The data will be sent from the local memory to a read-out board after the spill is over. In between spills, the electronics will record data from cosmic rays.

The QIEs and associated circuitry will be built on small daughter boards called MENU Modules, which resemble memory SIMMs. The boards contain a high density of surface mount parts. The MENU Modules plug in to a mother board called the MINDER Module. The MINDERs reside in front end crates called MINDER Crates, which are a semicustom design. There is a crate controller in the MINDER Crates called the KEEPER, which controls all activity in the crate. When data is acquired, it is stored on the MENU Modules. After data is acquired, the MINDER then initiates a readout operation, where the data is sent from the MENUs to a VME readout board, called the MASTER Module. The MASTER resides in a $9 \mathrm{U}$ VME crate located some distance away from the MINDER Crates. All of the board designs contain a high level of programmable logic to do the complex processing of data and control of operations.

The chip design, and the development of the QIE daughter board, are responsibilities of Fermilab. Argonne is responsible for the design the MASTER Module, the MINDER Module, the KEEPER, and the MINDER Crate. We also have overall responsibility for the design of the rest of the system for the Near Detector, including the specifications for the QIE performance.

The first prototype MASTER Module was designed and built in latter part of 2000 . In this period, we continued testing the MASTER. We also began the designs of the KEEPER and the MINDER. We plan to build prototypes by the fall of 2001. A small system test that includes all components of the system is planned for October, 2001.

ZEUS: Work continued at the ZEUS experiment at DESY to replace the tracking detector in the forward region during the shut down in 2000. The new detector will use straw tubes, rather than the older-style wire chamber technology. The detector produces a pulse in response to a charged particle passing through the detector. The front end electronics is situated directly on the detector. It receives charge pulses from the detector, and sends a digital signal to the "back end" electronics located off the detector in a counting room, where 
a timestamp for the signal is recorded. The back end processors then use the timestamps to reconstruct the trajectory of the particle through the tracking detector. There are $\sim 12,000$ channels in the detector in total, although the front end electronics multiplexes 6 detector channels into each readout channel to reduce the number of signal wires between the front end and the back end.

The front end electronics uses a custom integrated circuit designed at PENN, called the ASDQ. The chip was designed for use with the tracking detector in the CDF experiment, and performs the front end analog signal processing. We completed the prototype design and testing in the summer of 2000. Production began in the fall of 2000. The checkout was completed in February 2001, with all boards sent to DESY to be installed on the detector. We were successful in meeting the production and commissioning goals of the experiment.

(G. Drake)

\section{THEORETICAL PHYSICS PROGRAM}

\section{II.A. THEORY}

\section{II.A.1 Upsilon Decay to a Pair of Bottom Squarks}

In several theory papers, including Physical Review Letters 86 (2001) 4231-4234 (Ed Berger, Brian Harris, David Kaplan, Zack Sullivan, Tim Tait, and Carlos Wagner), it is suggested that the mass of the bottom squark may be quite small. If the bottom squark mass is less than the mass of one of the Upsilon (Y) states, then $\mathrm{Y}$ decay to a pair of bottom squarks might proceed with sufficient rate for experimental observation or exclusion of a light bottom squark. In a paper written in May 2001 with Louis Clavelli (U. Alabama), hepph/0105147, published in Physics Letters B512 (2001) 115-120, Ed Berger computes the expected rate for $\mathrm{Y}$ decay into a pair of bottom squarks as a function of the masses of the bottom squark and the gluino. The mass of the gluino enters because the gluino is exchanged in the decay subprocesses they compute. In their work, the electronic width of the $\mathrm{Y}$ is used as the source of absolute normalization.

The data sample is largest at the $\mathrm{Y}(4 S)$. For a fixed gluino mass of $14 \mathrm{GeV}$, they compute a branching fraction into a pair of bottom squarks about $10^{-3}$, for a bottom squark mass of $2.5 \mathrm{GeV}$, and about $10^{-4}$ for a bottom squark mass of 4.85 $\mathrm{GeV}$. A sample as large as 10,000 may be available in current data from runs of the CLEO detector. Members of the CLEO and BaBar collaborations have expressed strong interest in this work.

In their work, Berger and Clavelli also present calculations of decay rates in $\mathrm{Y}(1 S), \mathrm{Y}(2 S)$, and Y $(3 S)$ decays. Their results, shown in Fig. 1, can be read as predictions 
of the width for the corresponding values of bottom squark and gluino masses, or as lower limits on the sparticle masses given known bounds on the branching fractions. The current experimental uncertainties on the hadronic widths of the $\mathrm{Y}$ states are compatible with the range of values of the gluino and bottom squark masses favored in the work by Berger et al on the bottom quark production cross section in hadron reactions, cited above. The analysis of $\mathrm{Y}$ decays shows nevertheless that tighter experimental bounds on the bottom squark fraction are potentially powerful for the establishment of lower bounds on masses of the gluino and bottom squark.

A dedicated experimental investigation of $\mathrm{Y}$ decay into bottom squarks is warranted. Direct observation of the decay requires an understanding of the ways that bottom squarks may manifest themselves, a topic on which Berger is now working actively. If the bottom squark is relatively stable, it could pick up a light anti- $u$ or anti- $d$ quark and become a "mesino" with spin one-half, the superpartner of the $B$ meson. The mesino has baryon number zero but acts like a heavy anti-proton -- perhaps detectable with a time-of-flight apparatus or via $\mathrm{dE} / \mathrm{dx}$ measurements. Possible baryon-number-violating R-parity-violating decays of the bottom squark lead to $u+s ; c+d$; and $c+s$ final states. These final states of four light quarks might be distinguishable from conventional hadronic final states mediated by the three-gluon intermediate state.

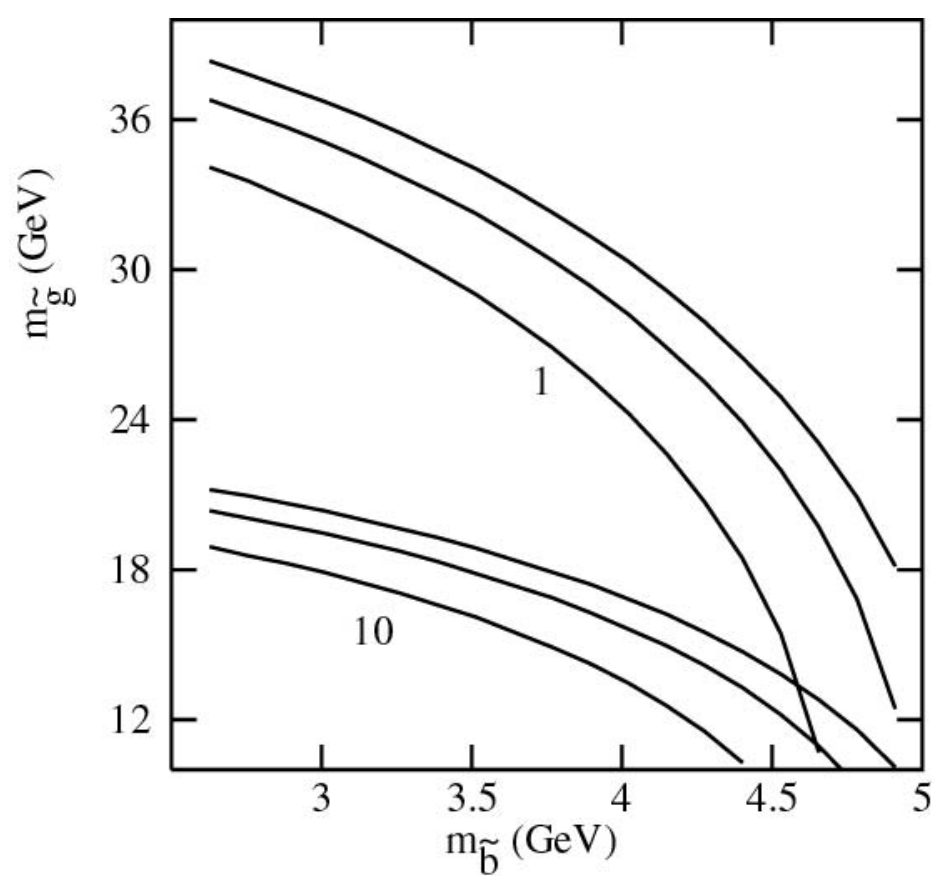

Fig. 1. Loci in the plane defined by the bottom squark and gluino masses, $m_{\tilde{b}}$ and $m_{\tilde{g}}$, for which the rate for decay into a pair of bottom squarks is either 10 times the leptonic width $\Gamma_{\bar{\ell} \bar{\ell}}$ for each $\mathrm{Y}(n S)$, with $n=1-3$ (lower set of curves) or equal to $\Gamma_{\bar{\ell} \bar{\ell}}$ (upper set). Above the curves, the rate would be less. Within each set of curves, the order is (bottom to top) $1 S, 2 S, 3 S$. 
A similar theoretical study may be carried out for decays of other bottomonium states into pairs of bottom squarks, another investigation on which Berger is embarked.

(E. L. Berger)

\section{II.A.2 Massive Lepton-Pair Production at Large Transverse Momentum}

The study of massive lepton-pair production in hadronic collisions (the Drell-Yan process) has been a valuable pursuit for many years. The process is an excellent laboratory for theoretical and experimental investigations of strong interaction dynamics, and it is a channel for discovery of quarkonium states and intermediate vector bosons. In prior years, along with Lionel Gordon and Michael Klasen, Ed Berger introduced a novel idea for extracting information on the gluon parton density from data on lepton-pair production in hadron reactions at large transverse momentum but relatively small values of mass of the pair.

In the region in which the transverse momentum can be much larger than the mass of the lepton pair, a crucial open question is the potential importance of terms that depend on the logarithm of the ratio of the transverse momentum and the mass. In the paper "QCD Factorized Drell-Yan Cross Section at Large Transverse Momentum”, hep-ph/0107309, accepted for publication in Physical Review D, Ed Berger, Jianwei Qiu, and Xiaofei Zhang (Iowa State U.) derive a new factorization formula in perturbative quantum chromodynamics for the Drell-Yan massive lepton-pair cross section as a function of the transverse momentum of the pair. When the transverse momentum is much larger than the pair's invariant mass, this factorization formula systematically resums the logarithmic contributions to all orders in the strong coupling. The authors show that resummation is quantitatively important when the collision energy is large and the transverse momentum is greater than the pair mass, and they argue that perturbative expansions are more stable and reliable in terms of the modified factorization formula. Berger et al's modified factorization formalism reorganizes the single perturbative expansion of conventional QCD factorization into two perturbative expansions plus perturbatively calculated parton-to-virtual photon fragmentation functions. The main advantage of this reorganization is that the new perturbative expansions are evaluated at a single hard scale and are free of large logarithmic terms.

Berger and collaborators present predictions for the cross sections for massive lepton-pair production at energies of interest for experiments at the Fermilab Tevatron, Brookhaven's RHIC, and the CERN Large Hadron Collider. They include both leading order and next-to-leading order direct short-distance contributions and the resummed logarithmic contributions. They confirm that after the large logarithmic terms are resummed to all orders in the strong coupling, the Drell-Yan cross section at large transverse momentum remains an excellent source of constraints on the gluon parton density.

(E. L. Berger) 


\section{II.A.3 Argonne 2001 Theory Institute on Supersymmetry and Extra Dimensions}

The Argonne 2001 Theory Institute on Supersymmetry and Extra Dimensions took place over the two-week period June 17 - 29, 2001. Edmond Berger, Gordon Chalmers, Brian Harris, David Kaplan, Zack Sullivan, Tim Tait, and Carlos Wagner organized it with the indispensable assistance of theory secretary Sandy Gotlund and computer support from Ron Rezmer. The goal was to examine outstanding issues in theory and phenomenology, as well as the impact of recent theoretical developments on searches for evidence of supersymmetry (SUSY) and extra space-time dimensions at the CERN LEP collider, the Fermilab Tevatron, the CERN Large Hadron Collider (LHC), and a future high energy electron-positron linear collider (LC). Three talks were scheduled per day. Intense informal discussion was facilitated and encouraged among external participants and Argonne physicists by the way the program was structured and by efforts to provide office space and computer access within the High Energy Physics building for all participants. The topics explored extended beyond the theory and phenomenology of SUSY and extra dimensions to include, inter alia, neutrino mass and mixing, Higgs production and decay, R-parity violation, CP-violation, rare B decays, and dark matter. Forty-seven theorists participated as well as a few experimenters. The Theory Institute was open to participation by all in the Division and elsewhere. Collaborations were originated, and the enthusiastic responses of participants included many suggestions for a return engagement in 2002. Associate Laboratory Director Frank Fradin provided funding through a Theory Institute grant of $\$ 20 \mathrm{~K}$. Proceedings were published electronically as http:/gate.hep.anl.gov/berger/anlsusy01/schedule.html. The electronic proceedings of the previous Argonne 2000 Theory Institute on Supersymmetry and Higgs Physics can be found at http://gate.hep.anl.gov/berger/anlsusy2k/sched.html.

(E. L. Berger)

\section{II.A.4 Compatibility of Approaches to Heavy-Quark Fragmentation}

This research was described in the report for July 1--December 31, 2000. A paper on it (ANL-HEP-PR-00-093, hep-ph/0012037) has now been published in Phys. Rev. D 63, 077503 (2001).

(G. T. Bodwin)

\section{II.A.5. Resummation of Large Corrections to Heavy-Quarkonium Decays}

This research was described in the report for January 1--June 30, 2000. During the current reporting period, a lengthy paper on it (ANL-HEP-PR-00-109, hep-ph/0106095) was written and submitted to Physical Review A.

(G. T. Bodwin) 


\section{II.A.6 Computational Physics}

The major new project started in this period was a study of lattice QCD at finite isospin density. Nuclear matter not only has a finite baryon number density, but also (due to coulomb repulsion) a finite (negative) isospin $\left(I_{3}\right)$ density. Whereas including a chemical potential for baryon number leads to a complex fermion determinant and cannot be handled by current simulation methods, including a chemical potential, $\mu_{I}$ for the third component of isospin yields a real non-negative determinant, which is rendered positive by the inclusion of the small symmetry-breaking term needed to observe spontaneous symmetry breaking on a finite lattice. Preliminary indications from our simulations on an $8^{4}$ lattice suggest that there is some critical value of $\mu_{I}$ above which a charged pion condensate forms that spontaneously breaks the third component of isospin and parity (Fig. 1). Considering this theory at finite temperature indicates that for large enough temperature, this condensate evapourates at a first order phase transition.

We have continued our studies of 2-colour lattice QCD with a finite chemical potential, $\mu$, for quark number. We completed our simulations at a relatively large quark mass on $8^{4}$ and $12^{3} \times 24$ lattices, which give clear evidence for a second order phase transition at $\mu=\mu_{c} \sim m_{\pi} / 2$, to a superfluid state characterized by a diquark condensate that spontaneously breaks quark number. We have also observed the diquark Goldstone boson associated with this breaking. Note that true (3-colour) QCD is also expected to form a diquark condensate at large baryon-number density. However, because this condensate is, of necessity, coloured, the symmetry breaking should be realized in the Higgs mode and the system is a colour superconductor. We also observed the restoration of quark-number symmetry at high temperature at a first order transition. These calculations are being extended to smaller quark mass.

Other projects which were ongoing during this period included continuation of our finite temperature simulations of lattice QCD with an action that includes a chiral 4-fermion interaction allowing simulations at zero quark mass, on an $N_{\mathrm{t}}=4$ lattice. This should be able to determine whether the deconfinement phase transition is first or second order on this size lattice. We are also continuing our investigation of the chiral properties of domain-wall fermions in the quark-gluon plasma phase of lattice QCD.

The simulations described above were performed on the IBM SP's at NERSC and NPACI, and on the Cray SV1's also at NERSC. 


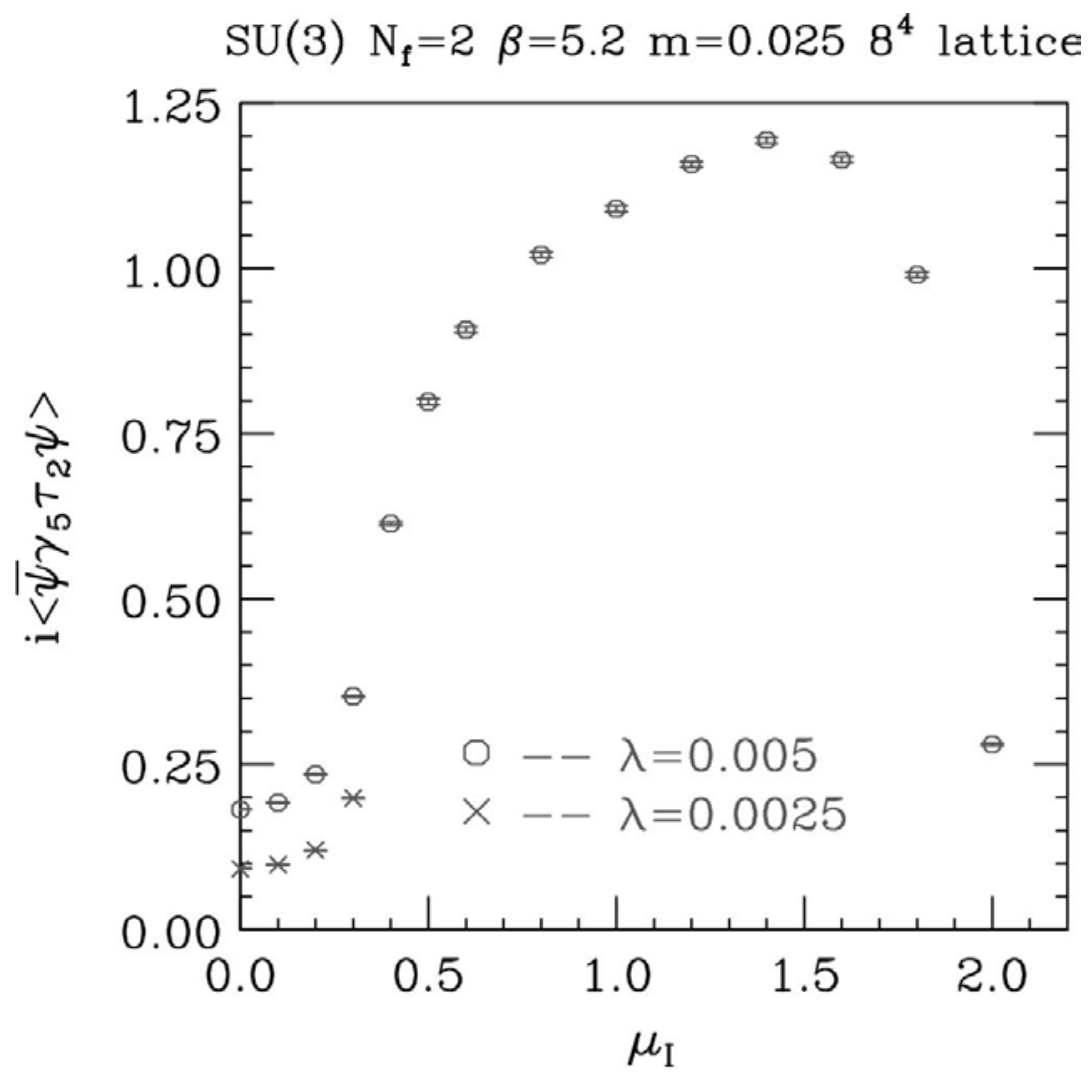

Figure 1: $\quad \mu$ dependence of the pion condensate.

(D. K. Sinclair)

\section{II.A.7 Brane Effects on Large Extra Dimension Scenarios}

The Standard Model of particle physics provides an excellent explanation of all quantities measured experimentally. Within this model, particles acquire mass as a result of the breakdown of the electroweak gauge symmetry and, therefore, all masses are of the order of the electroweak symmetry breaking scale. This scale is governed by the Higgs mass parameter, which receives quadratic divergent corrections in perturbation theory. Because the Standard Model provides a consistent description of particle interactions up to the Planck scale at which gravity becomes relevant, the question is why the value of the Higgs mass parameter is so much smaller (sixteen orders of magnitude smaller) than the Planck scale. Particle physics explanations of the hierarchy of mass scales always relied on new physics, which modified the high energy behaviour of the Higgs mass parameter, either by canceling the quadratic divergences or by making the Higgs a composite particle.

Recently, a new solution was proposed by assuming that the fundamental Planck scale is not large, as naively determined by Newton's Law, but it is actually of the order of the electroweak symmetry breaking scale. Because the strength of gravity interactions is proportional to the inverse of the square of the Planck scale, this would lead to gravity 
interactions much stronger than what is measured experimentally. In this new proposal, however, the weakness of gravity is explained by assuming that we (quarks, leptons and gauge bosons) live in a four-dimensional domain wall (brane) located in a higher dimensional space, in which only gravity can propagate. The apparent weakness of gravity is then explained by the small overlap of the graviton wave function with the one of the matter and gauge fields. The fundamental Planck scale $M$ is related to the scale $M_{P l}$ observed in four-dimensional experiments by

$$
M_{P l}^{2}=M^{2+n} V_{n}
$$

where $n$ is the number of extra dimensions and $V_{n}$ is the volume of the extra dimensional space.

From the above relation, it is quite clear that if $M \cong O 1 \mathrm{TeV}$, then the radius, $R$, of the extra dimensions should be large, in order to obtain the right $M_{P l}$ value. Actually, for $n=1, R$ is too large to be reconciled with the observable macroscopic properties of gravity. For $n \geq 2, R$ is in the submillimeter regime, at which the behaviour of gravity has not been tested experimentally.

Gravitons propagating into the extra dimensions will see their dispersion relation modified by the introduction of a mismatch between their energy and their four-dimensional momentum. This mismatch can be reinterpreted as a mass from the four-dimensional point of view. Because the momentum in the extra dimensions is quantized in units of $\pi / R$, a four-dimensional observer will see many different gravitons, with quantized masses, the socalled Kaluza-Klein (KK) graviton states. An experimental signature of the above-described scenarios will be the production of missing energy, associated with the KK graviton states, in high energy colliders.

The above framework still allows for the presence of a local four-dimensional Einstein term on the brane, different from the one associated with the fundamental Planck scale in the extra-dimensional space. Indeed, such a term will always be generated by quantum corrections. In a recent article published in Nucl. Phys. B 609, 499 (2001), I have investigated the possible impact of this term on the properties of the KK gravitons. This local Einstein term induces a kinetic mixing of the KK gravitons, which modifies their kinetic relations. Denoting $\bar{M}$ to the mass scale associated with this term, one can define a critical scale,

$$
M_{c}=\frac{M^{2}}{\bar{M}}
$$

One can prove that $M_{P l}^{2}=\bar{M}^{2}+M^{2+n} V_{n}$. The presence of $\bar{M}$ implies that $\mathrm{KK}$ gravitons with masses larger than $M_{c}$ couple very weakly to matter, while KK gravitons 
with masses smaller than this value couple in the standard way. A graviton of mass of order $M_{c}$ appears in the spectrum and couples with strength proportional to $1 / \bar{M}^{2}$. Two possibilities may occur. If $M_{c}$ is smaller than $1 / R$, the $\mathrm{KK}$ gravitons decouple from matter and one has a tale of two gravitons; the standard graviton governing the gravity interactions up to scales of order $1 / M_{c}$, while the new gravitons of mass $M_{c}$ governing the gravity interactions at scales smaller than that. The effective Planck scale would be modified from $M_{P l}$ to $\bar{M}$ at energy scales of order $1 / M_{c}$. This behaviour may be tested in table top experiments.

Under the presence of the two graviton states, the gravity potential reads

$$
V(r)=-G_{N} \frac{m_{1} m_{2}}{r}\left(1+\alpha e^{-r / \lambda}\right)
$$

where the parameters $\alpha$ and $\lambda$ are defined by

$$
\alpha=\frac{M_{P l}^{2}}{\bar{M}^{2}}, \quad \lambda=M_{c}^{-1},
$$

In Fig. 1, we present the regions of parameter space that can be probed experimentally by present and future planned experiments. 


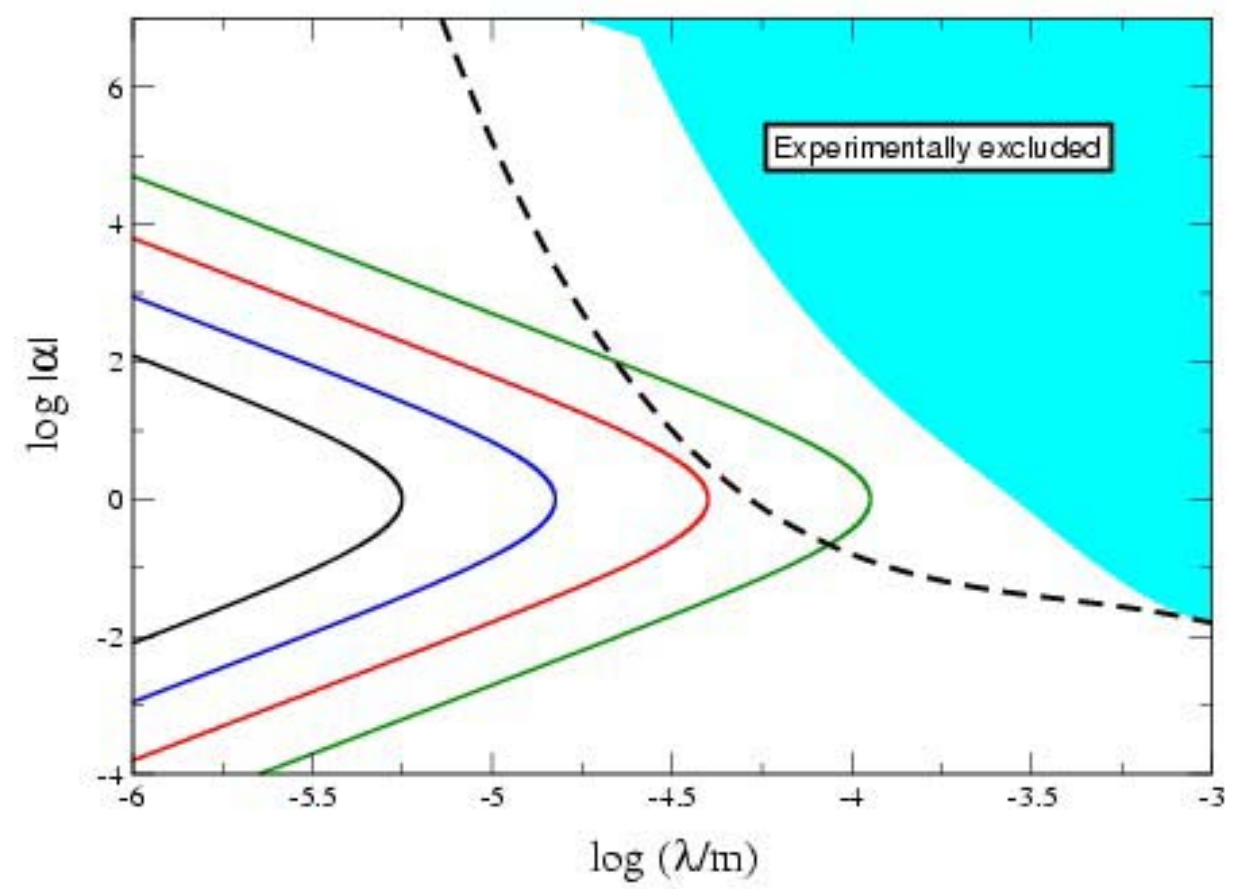

Figure 1. Comparison with table-top experiments. Solid curves are for $d=3,4,5$, 6, from top to bottom, respectively, and $M=1 \mathrm{TeV}$. The shaded region is excluded from present experimental data, while the dashed curve is the projected experimental sensitivity.

If $M_{\mathrm{c}}$ is larger than $1 / R$ instead, the presence of $\bar{M}$ modifies the missing energy signatures, by providing a cutoff for the amount of KK graviton states that may be emitted. If $\bar{M}$ is not much larger than the electroweak scale, it could even be possible to observe a resonance of mass $M_{\mathrm{c}}$ and coupling proportional to $1 / \bar{M}^{2}$. The most important characteristic of this resonant state will be its spin, $S=2$, which can be easily tested experimentally.

(C. Wagner)

\section{II.A.8 The Chiral Anomaly and the Regge Limit Of QCD}

For some time, Alan White has pursued a program designed to derive the regge limit of QCD from gluon and quark regge on diagrams. The non-perturbative features of confinement and chiral symmetry breaking are anticipated to arise from the combination of mass-less, infrared divergences with anomalous, reggeon interactions involving the triangle anomaly. In a newly published paper, Phys. Rev. D 63, 016007 (2001) (52 pages), a first step towards demonstrating the existence of the anomalous interactions is taken. It is shown that the infrared divergence, associated with the triangle graph axial anomaly, can occur in 
triple-regge multi-reggeon interactions due to non-physical asymptotic triple discontinuities. In a new paper (hep-ph/0104128), an asymptotic discontinuity analysis is applied to highorder Feynman diagrams to show that the anomaly does indeed exist in contributions to the triple-regge nine-reggeon interaction. This implies that the anomaly occurs in the interactions of reggeon states that have the quantum numbers of the anomaly current and establishes a direct connection with the well-known U(1) problem.

Most recently, White has found a new starting point for the general program. It is well-known that when a chiral symmetry is broken spontaneously, the chiral flavor anomaly requires that (in a special kinematic configuration) a Goldstone boson pole must appear in the vertices for the corresponding axial current to interact with pairs of vector currents. Starting from this property, it can be shown that when $\mathrm{SU}(3)$ color is broken to $\mathrm{SU}(2)$, the flavor anomaly provides (infinite momentum) wee-gluon couplings for Goldstones. As a result, Goldstones have just the wee gluon component that White's general program implies all physical states must possess. The Goldstone states are quark/antiquark "pions" and, also, because of the reduced gauge symmetry, quark/quark (or antiquark/antiquark) "nucleons". The pion amplitude can be extracted from a four-current amplitude. The wee gluon component produced by the flavor anomaly combines with $\mathrm{U}(1)$ anomaly reggeon interactions to produce an infrared divergence (factorized off as a condensate) that selects infinite momentum physical amplitudes (containing the exchanged "pomeron") very much, as in the reggeon diagram analysis. The pion amplitude is a "partonic" scattering within the massive part of the gauge group, but with zero-momentum quarks (from the U(1) anomaly) injecting color and spin-dependence into massive reggeized gluon interactions.

In the new approach, it is clear that the physical pomeron couples only to states that have a component that is a Goldstone boson when SU(3) color is broken to SU(2)--this includes both pions and nucleons. The physical distinction between the BFKL pomeron and White's pomeron is thus established, as is the very important result that stable hadron states are all (either directly or indirectly) Goldstone bosons. The new approach is to be presented at upcoming, European conferences.

(A. R. White)

\section{II.A.9 Negative Probability and Uncertainty Relations}

C Zachos has made progress in a long-term project on Deformation Quantization, which underlies noncommutative field theories, currently informing open (tachyonic) string physics. In collaboration with T Curtright (U of Miami) and based on their recent analysis, J. Math. Phys. 42, 2396-2415 (2001), they have invented a concise derivation of all uncertainty relations (hep-th/0105226, NL-HEP-PR-01-034) entirely within the context of phase-space quantization, without recourse to operator methods, to the direct use of Weyl's correspondence, or to marginal distributions of $x$ and $p$. 
For classical (non-negative) probability distributions, expectation values of nonnegative functions are likewise non-negative, and thus result in standard constraint inequalities for the constituent pieces of such functions. On the other hand, in phase-space quantization, the distribution functions are non-positive-definite, such as, in general, the quasi-probability Wigner Function: it was interpreted early on by Bartlett and Feynman as a "negative probability function", with the proper non-negative marginal probabilities upon projection to either $x$ or $p$ space. A fifty-year-old gap in the phase-space quantization literature is summarized by the question: "How does Heisenberg's standard quantum mechanical uncertainty relation arise for moments of such distributions?" i.e. how do expectation values of (c-number) observables constrain themselves with $\hbar$ entering into the outcome, when the variables $x, p$ do not contain $\hbar$ ? How do their moments manage to extract $\hbar$ out of the Wigner function?

The answer was found to lie in Groenewold's associative *-product, which is the cornerstone of phase-space quantization: Expectation values of arbitrary real * -squares were shown to be positive semi-definite - even though the Wigner distribution $f$ $(x, p)$ itself is not, and thus expectation values of arbitrary squares are not. In the proof, no operators occur ( $x$ and $p$ are c-numbers), beyond the $*$-product operation.

The technique developed may be applied to general observable quantities of arbitrary size and complexity, in principle, and uncertainty bounds are saturated through the solution of suitable, but difficult, *-functional equations.

(C. Zachos)

\section{ACCELERATOR RESEARCH AND DEVELOPMENT}

III.A. ARGONNE WAKEFIELD ACCELERATOR PROGRAM

\section{III.A.1. The Argonne Wakefield Accelerator Status}

During this period, the new AWA gun was completed fabricated and installed on the gun test stand. We have conducted the bead pull experiment and found that the fields are balanced within $1 \%$ as shown in Figure 1. The high power RF commissioning is underway. During this period, with additional funding from DOE and lab internals, we have specified and purchased a new all solid state laser system for the old AWA laser replacement. This laser is expected to be delivered in early next year. In order to compensate the new gun requirement, we have also extended the RF pulse length from $4 \mu \mathrm{s}$ to $6.5 \mu$ s by putting additional capacitor banks in the modulator. 


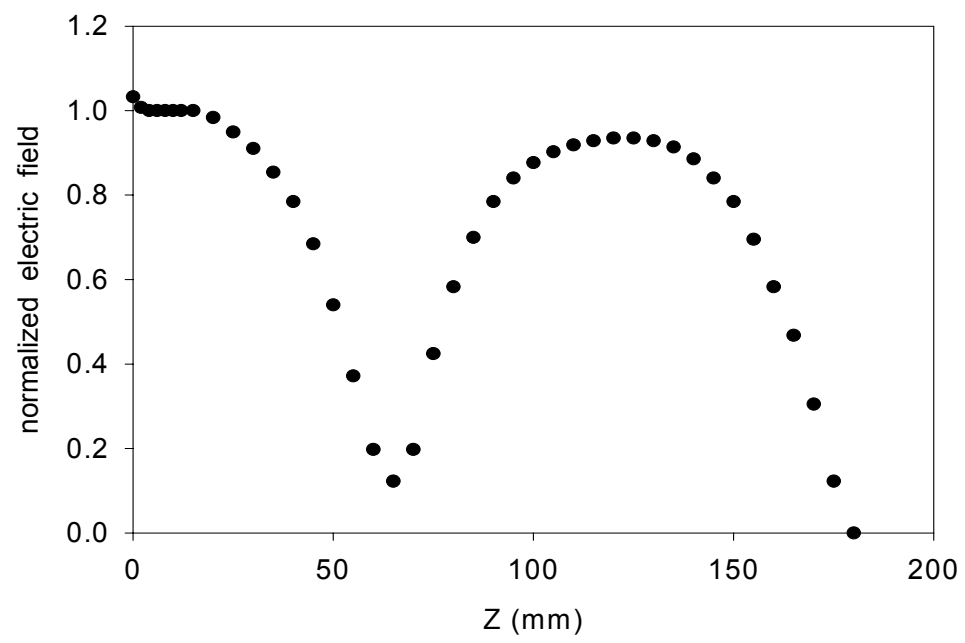

Figure 1: Bead pull measurement of electrical field balance in the new AWA 1-1/2 cell gun. It showed less than $1 \%$ difference between the two cells.

\section{III.A.2. The AWA Experiments}

a. We have continued two-beam acceleration experiment, with a newly installed laser trombone. The previous experimental data were confirmed. However, we found that a relative high energy fluctuations of the witness beam which prevent us to perform precision measurement. Currently we are in the process in identifying the source of this fluctuations.

b. A new experiment to study transformer ratio enhancement in collinear Wakefield accelerator was designed. The goal is to demonstrate physics aspects of high transformer ratio $(>2)$ in collinear Wakefield scheme. It is critical for practical application of collinear WF method to linear collider concept. We are planning to use 40 precisely machined copper cavities operating at $13.65 \mathrm{GHz}$ as a Wakefield device. Then we use a ramped triangle bunch train to drive the Wakefield device, an idea developed at ANL working with A. Kanareykin (St. Petersburg), one can obtain transformer ratio as high as $2 \mathrm{X}$ the bunch train numbers. In our designed experiment, we are expecting to observe $\mathrm{R}>6$. The Wakefield device has been order from an external vendor and to be shipped to us in a few months.

\section{III.A.3. New Accelerator Concept Developed.}

During this period, we have investigated a partially dielectric loaded rectangular waveguide as accelerator structure. This type of structure has interesting properties as follows: 
a. Can be easily tuned to correct fabrication error by tuning the metallic side walls,

b. Can store more energy than cylindrical tube thus reduce the beam loading (important at high frequencies, as pointed out by many)

c. Acceleration field is not uniform across the transverse dimension; the field acts on the beam like a RF quadrupoles while beam accelerated.

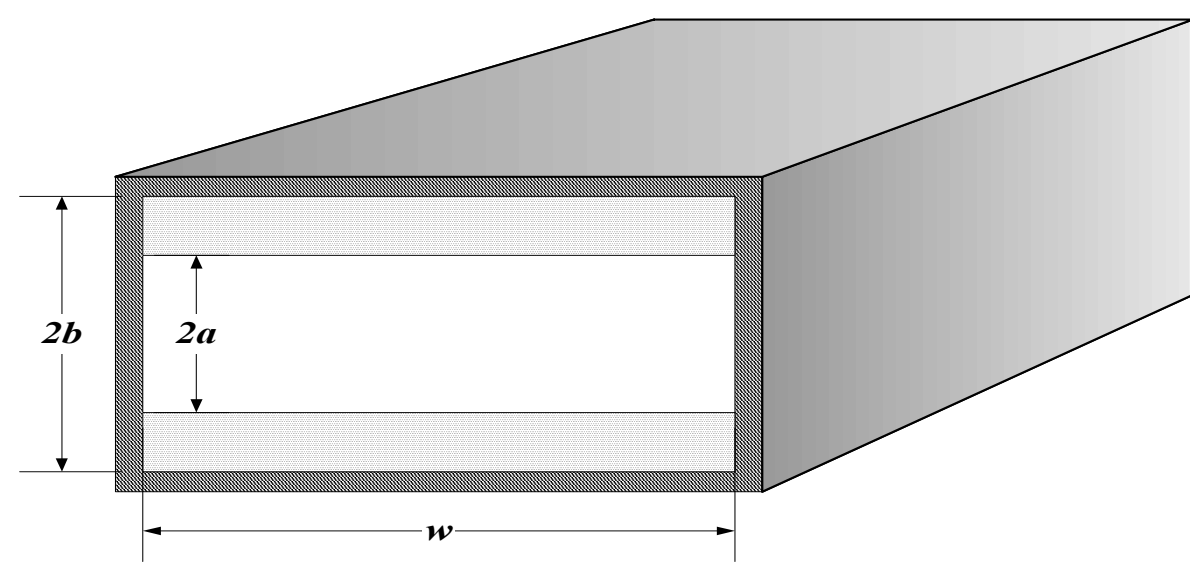

Figure 2: A partial dielectric loaded waveguide as an acceleration structure.

Thus far, we have obtained analytical expression for fields, Wakefields, R/Q and all other accelerator properties. The results are in very good agreement with simulations done with the MAFIA. A set of dielectrics is ordered and to be tested in the lab in the near future. Summary of this work has been submitted for publication in Phy. Rev. E.

\section{III.A.4. Design of precision Wakefield measurement system for Engineering Test Facility (ETF) at Fermilab}

We have proposed a precision Wakefield measurement system for Engineering Test Facility (ETF) at Fermi Lab. As shown in the Figure 3, this proposed system would have resolution of 100 times more than ASSET. A joint paper was published with FNAL/SLAC on this topic. 


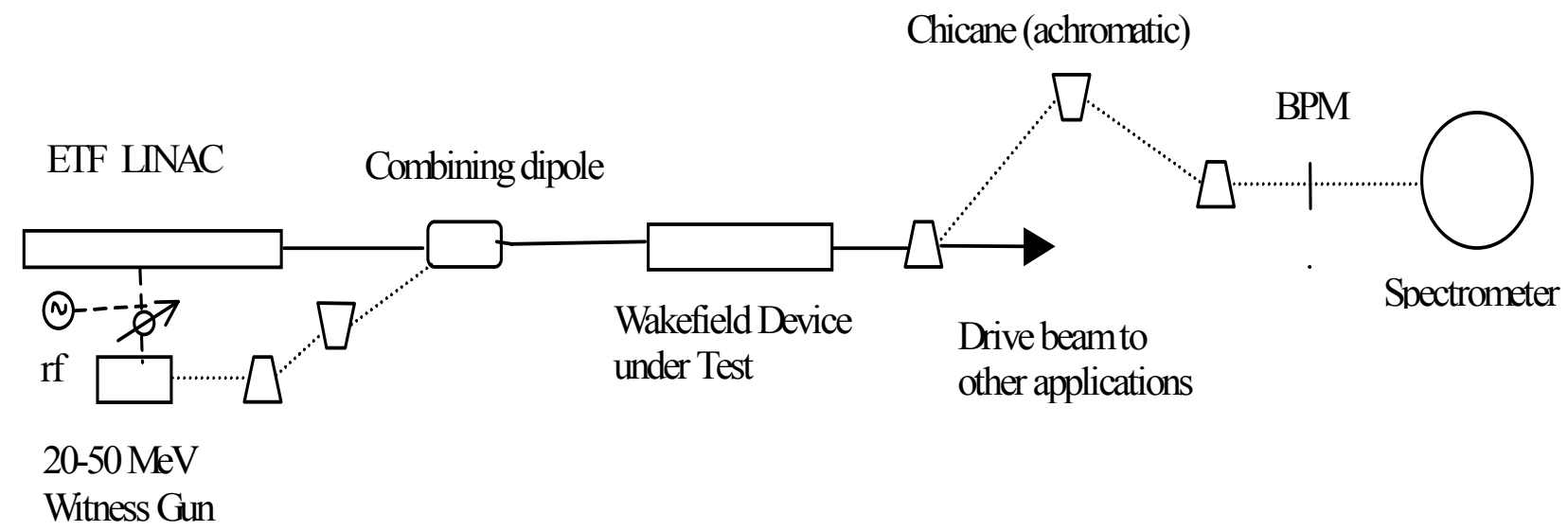

Figure 3: Schematic diagram of the proposed Wakefield measurement system for ETF at FNAL.

(W. Gai)

\section{B. MUON COLLIDER AND NEUTRINO SOURCE}

\section{a) Instrumentation for the Neutrino Source}

Study 11, A Brookhaven site dependent Neutrino Source Design Study Is intended to be a second iteration design, with more neutrino intensity, more design detail and less project cost. In this study, Argonne effort considered all aspects of beam instrumentation were considered: target, decay solenoid, buncher, cooling line, accelerator systems and storage ring with the aim of identifying problems and costs of each. Eventually this involved proposing specific methods that would be used for beam diagnostics in each element and estimating the costs and any $R \& D$ requirements. The aim was to show that although there were a number of new and troublesome problems, standard beam diagnostics were sufficient to operate the machine. This was in general true, however there were problems measuring the direction and size of the neutrino beam coming out of the storage ring with sufficient accuracy. This was solved with the use of a high rate neutrino detector very close to the production straight section which could distinguish decays in the production straight from those in the coupling sections.

While much of the instrumentation required for the neutrino source was common to high energy physics, it turns out that instrumentation for both the beam on target and the use of high energy beams with liquid hydrogen targets are not well understood. A simple prototype Schlieren system was built which could demonstrate slow quiescent flow of water in a tank, a prototype of a high energy beam in a liquid $\mathrm{H}_{2}$ target, and with Lauren Ducas a 
summer student from the Univ. of Illinois, of target heating using a $20 \mathrm{MeV}$ electron beam at the ANL/CHM linac. Further work is proceeding.

\section{b) An $\mathrm{e}^{+} \mathrm{e}^{-}$Collider in the VLHC Tunnel}

This has been a continuing interest since the last Snowmass meeting in 1996. The only estimate of larger $\mathrm{e}^{+} \mathrm{e}^{-}$collider costs was done in 1975 by Richter, and most of his assumptions are not relevant to machines larger than LEP. A detailed study to look at a design started in about October of last year with Tanaji Sen of Fermilab,. We then discovered that Alvin Tollestrup of Fermilab was interested in this option and had been also interested in looking for a more complete design. Alvin, Gerry Dugan of Cornell, and I organized another workshop at IIT in March, again sponsored by the Illinois Consortium on Accelerator Research, which looked at the issues and design of such a machine, with the aim of producing a parameter list with critical issues that could be used as a basis for discussion at Snowmass. The workshop gained considerably from very active participation from a number of senior CERN/LEP staff. We did produce this report and have been continuously finding ways of upgrading the luminosity and lowering the cost. The machine parameters are described in a number of papers. This option seems superior in many respects to the linear collider up to $500 \mathrm{GeV}$ in the c.m.,(more luminosity, much better energy resolution, more stable and reliable, lower cost) and an upgrade path to the VLHC at higher energies.

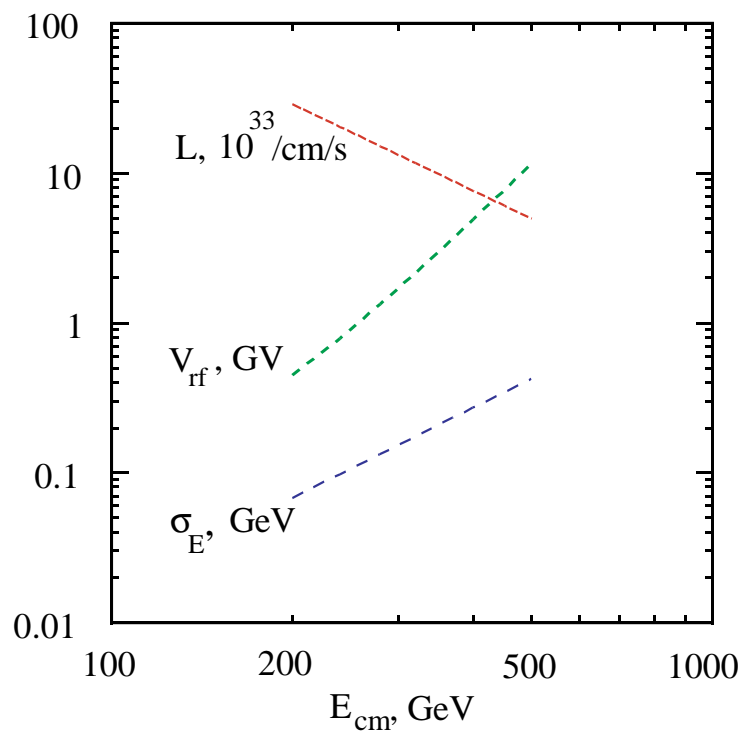

Figure 1: The parameters of a large circular collider in a VLHC tunnel.

The Argonne part of this work involved design of a first iteration magnet / vacuum chamber assembly able to cope with the small divergence synchrotron radiation beams whose transmission varies over a wide range as the energy is increased from an injection energy of 
$40 \mathrm{GeV}$ to an operating energy of $200-250 \mathrm{GeV}$. A detailed paper describing the design issues was submitted to Phys. Rev, Special Topics with Tanaji Sen of Fermilab, together with shorter papers presented at Snowmass, the Chicago PAC conference and the workshop proceedings.

(J. Norem) 


\section{PUBLICATIONS}

\section{A. BOOKS, JOURNALS AND CONFERENCE PROCEEDINGS}

A Survey of Star Product Geometry

C. Zachos

In: NATO Advanced Research Workshop Science Series: Integrable Hierarchies and Modern Physical Theories, edited by H. Aratyn and A. Sorin (Kluwer-AP, Dordrecht, 2001) pp. 423-435.

$b \rightarrow s \gamma$ and Supersymmetry with Large $\tan \beta$

M. Carena, D. Garcia, U. Nierste, and C.E.M. Wagner

Phys. Lett. B 499, 141-146, (2001).

Compatibility of Various Approaches to Heavy-Quark Fragmentation

G. T. Bodwin and B. W. Harris

Phys. Rev. D 63, 077503, (March 2001).

Direct Probes of R-Parity-Violating Supersymmetric Couplings Via Single-Top-Squark

Production

E. L. Berger, B. W. Harris, and Z. Sullivan

Phys. Rev. D 63, 115001, (April 2001).

Double Diffraction Dissocaition at the Fermilab Tevatron Collider

R. E. Blair, K. L. Byrum, E. Kovacs, S. E. Kuhlmann, T. LeCompte, L. Nodulman,

J. Proudfoot, R. Thurman-Keup, R. G. Wagner, A. B. Wicklund

Phys. Rev. Lett., Vol. 87, 141802, (2001).

Do Electroweak Precision Data and Higgs-Mass Constraints Rule Out a Scalar Bottom Quark with Mass of Order $5 \mathrm{GeV}$

M. Carena, S. Heinemeyer, C.E.M. Wagner, and G. Weiglein

Phys. Rev. Letts. 86, 4463-4466, (May 2001).

First Measurement of the Ration B $(t \rightarrow W b / B(t \rightarrow W q)$ and Associated Limit on the CKM Element $\left|V_{t b}\right|$

R. E. Blair, K. L. Byrum, E. Kovacs, S. E. Kuhlmann, T. LeCompte, L. Nodulman,

J. Proudfoot, R. Thurman-Keup, R. G. Wagner, A. B. Wicklund

Phys. Rev. Lett., Vol. 86, 3233, (2001).

Generating All Wigner Functions

T. Curtright, T. Uematsu, and C. Zachos

J. Math. Phys. 42, 2396-2415 (June 2001).

Low-Energy Supersymmetry and the Tevatron Bottom-Quark Cross Section

E. L. Berger, B. W. Harris, D. E. Kaplan, Z. Sullivan, T.M.P. Tait, and C.E.M. Wagner Phys. Rev. D 63, 115001, (April 2001). 
Measurement of the Neutral Current Cross Section and $\mathrm{F}_{2}$ Structure Function for Deep Inelastic $\mathrm{e}+\mathrm{p}$ Scattering at HERA

S. Chekanov, M. Derrick, D. Krakauer, S. Magill, B. Musgrave, A. Pellegrino, J. Repond, R. Stanek, R. Yoshida

Eurp. Phys. Journal, Vol. C 21, 443-471, (May 2001).

Measurement of $K^{-} p \rightarrow \eta \Lambda$ Near Threshold

C. E. Allgower and H. Spinka, A. Starostin et al,

Phys. Rev., Vol. C 64, 055205, (2001).

Measurement of the $t \bar{t}$ Production Cross Section in $p \bar{p}$ Collisions at $\sqrt{s}=1.8 \mathrm{TeV}$

R. E. Blair, K L. Byrum, E. Kovacs, S. E. Kuhlmann, T. LeCompte, L. Nodulman, J. Proudfoot, R. Thurman-Keup, R. G. Wagner, A. B. Wicklund

Phys. Rev. D, Vol. 64, 032002, (2001).

Mid-rapidity anti-proton to proton ratio from $\mathrm{Au}+\mathrm{Au}$ collisions at $\sqrt{s_{N N}}=130 \mathrm{GeV}$

K. Krueger, T. LeCompte, H. M. Spinka, D. G. Underwood, A. Yokosawa

C. Adler et al.,

Phys. Rev. Lett., Vol. 86, 4778-4782, (May, 2001).

Multiplicity Distribution and Spectra of Negatively Charged Hadrons in $\mathrm{Au}+\mathrm{Au}$ Collisions at $\sqrt{S_{N N}}=130 \mathrm{GeV}$

K. Krueger, T. LeCompte, H. M. Spinka, D. G. Underwood, A, Yokosawa,C. Alder et al.,

Phys. Rev. Lett., Vol. 87, 112303, (June, 2001).

Multiplicity Moments in Deep Inelastic Scattering at HERA

S. Chekanov, M. Derrick, D. Krakauer, S. Magill, B. Musgrave, A. Pellegrino,

J. Repond, R. Stanek, R. Yoshida

Physics Lett. B, Vol. 510, 26-54, (April, 2001).

Open String Decoupling and Tachyon Condensation

G. Chalmers

JHEP 0106:012, (June 2001).

Search for Narrow Diphoton Resonaces and for $\gamma \gamma+W / Z$ Signatures in $p \bar{p}$ Collisions at $\sqrt{s}=1.8 \mathrm{TeV}$

R. E. Blair, K. L. Byrum, E. Kovacs, S. E. Kuhlmann, T. LeCompte, L. Nodulman, J. Proudfoot, R. Thurman-Keup, R. G. Wagner, A. B. Wicklund

Phys. Rev. D, Vol 64, 092002, (2001).

Simplifying Algebra in Feynman Graphs. Part III: Massive Vectors

G. Chalmers and W. Siegel

Phys. Rev. D 63,125027, (June 2001). 
Single Top Quark Production as a Window to Physics Beyond the Standard Model

T.M.P. Tait and C.-P. Yuan

Phys. Rev. D 63, 014018, (January 2001).

Study of the Effective Transverse Momentum of Partons in the Pronton Using Prompt Photons in Photoproduction at HERA

S. Chekanov, M. Derrick, D. Krakauer, S. Magill, B. Musgrave, A. Pellegrino,

J. Repond, R. Stanek, R. Yoshida

Physics Letters B, Vol. 511, 19-32, (March, 2001).

Supersymmetric CP-Violating Currents and Electroweak Baryogenesis

M. Carena, J. M. Moreno, M. Quiros, M. Seco, and C.E.M. Wagner

Nucl. Phys. B 599, 158-184, (2001).

Supersymmetry Contribution to Bottom Quark Production at Hadron Colliders

E. L. Berger

In: Proceedings of the 4th International Workshop on B Physics and CP

Violation (BCP4), edited by T. Ohshima and A. I. Sanda (World Scientific, Singapore, April 2001) pp. 221-224.

Suppression of Multi-Gluon Fluctuations in Jets at a Linear Collider

S. Chekanov

Physics Lett., B 509, 74-80 (2001).

The Triangle Anomaly in Triple-Regge Limits

A. R. White

Phys. Rev. D 63, 016007 (January 2001).

Variable Flavor Number Schemes Versus Fixed Order Perturbation Theory for Charm Quark Electroproduction

A. Chuvakin, J. Smith, and B. W. Harris

Eur. Phys. J. C 18, 547-553, (February 2001).

Viable Ultraviolet-Insensitive Supersymmetry Breaking

N. Arkani-Hamed, D. E. Kaplan, H. Murayama, and Y. Nomura

JHEP 0102:041 (February 2001).

\section{IV.B. MAJOR ARTICLES SUBMITTED FOR PUBLICATION}

A Hybrid Dielectric and Iris Loaded Periodic Accelarating Structure

Peng Zou, Liling Xiao, Xiang Sun, Wei Gai (ANL); Thomas Wong (IIT)

ANL-HEP-PR-01-008

Analysis of Low Energy AGS Polarimeter Data and Potential Consequences for PHIC Spin Physics

R. Cadman, K. Krueger, H. Spinka, D. Underwood, A. Yokosawa

ANL-HEP-PR-01-036 
Axions and a Gauged Peccei-Quinn Symmetry

H.-C. Cheng and D. E. Kaplan

Phys. Rev. Letts.

ANL-HEP-PR-01-021

Bottomonium Decay Matrix Elements from Lattice QCD with Two Light Quarks

G. T. Bodwin, D. K. Sinclair, and S. Kim

Phys. Rev. D

ANL-HEP-PR-01-026

Brane Effects on Extra Dimensional Scenarios: A Tale of Two Gravitons

M. Carena, A. Delgado, J. Lykken, S. Pokorski, M. Quiros, and C.E.M. Wagner

Nucl. Phys. B

ANL-HEP-PR-01-007

Chirality Violation in the QCD High-Energy S-Matrix

A. R. White

Phys. Rev. D

ANL-HEP-PR-01-017

Cosmological Constant in Broken Maximal Sugras

G. Chalmers

Phys. Rev. D

ANL-HEP-PR-01-020

Deconstructing Gaugino Mediation

H.-C. Cheng, D. E. Kaplan, M. Schmaltz, and W. Skiba

Phys. Letts. B

ANL-HEP-PR-01-046

Determination of the Quadratic Slope Parameter in $\eta \rightarrow 3 \pi^{\circ}$ Decay

C. E. Allgower, H. Spinka

W.B. Tippens et al. Phys. Rev. Lett., Vol. 87 , (Nov., 2001).

ANL-HEP-PR-01-024

Diquark Condensation at Nonzero Chemical Potential and Temperature

J. B. Kogut, D. Toublan, and D. K. Sinclair

Phys. Letts.

ANL-HEP-PR-01-027

Field Analysis of a Dielectric-Loaded Rectangular Waveguide Accelerating Structure

Liling Xiao, Wei Gai, Xiang Sun

Phys. Rev. E, "Physics of Beams" Section

ANL-HEP-PR-01-018 
First Measurement of the Ration B $(t \rightarrow W b) / B(t \rightarrow W q)$ and Associated Limit on the CKM Element $\left|V_{t b}\right|$

R. E. Blair, K. L. Byrum, E. Kovacs, S. E. Kuhlmann, T. LeCompte, L. Nodulman, J. Proudfoot, R. Thurman-Keup, R. G. Wagner, A. B. Wicklund ANL-HEP-PR-01-003

M-Theory and Automorphic Scattering

G. Chalmers

Phys. Rev. D D64:046014, (Aug., 2001).

ANL-HEP-PR-01-025

Massive Selfdual Perturbed Gauge Theory

G. Chalmers

Phys. Rev. D

ANL-HEP-PR-01-016

Measurement of Dijet Production in Neutral Current Deep Inelastic Scattering at High $\mathrm{Q}_{2}$ and determination of $\alpha_{s}$

J. Breitweg, S. Chekanov, M. Derrick, D. Krakauer, S. Magill, B. Musgrave, A.

Pellegrino, J. Repond, R. Stanek, R. Yoshida and the ZEUS Collaboration

Physics Lett., B, (May 2001).

ANL-HEP-PR-01-019

Measurement of Inclusive Antiprotons from $\mathrm{Au}+\mathrm{Au}$ Collisions at $\sqrt{s_{N N}=130} \mathrm{GeV}$

R. V. Cadman, K. Krueger, T. LeCompte, H. M. Spinka, D. G. Underwood,

A. Yokosawa

Phys. Rev. Lett., Vol. 87, 262302, (Oct. 2001).

ANL-HEP-PR-01-108

Measurement of the Inclusive Jet Cross Section in $p \bar{p}$ Collisions at $\sqrt{s}_{\mathrm{s}}=1.8 \mathrm{TeV}$

R. E. Blair, K. L. Byrum, E. Kovacs, S. E. Kuhlmann, T. LeCompte, L. Nodulman,

J. Proudfoot, T. Thurman-Keup, R. G. Wagner, A. B. Wicklund

Phys. Rev. D, Vol. 64, 0232001 (Oct. 2001).

ANL-HEP-PR-01-014

Measurement of Open Beauty Production in Photoproduction at HERA

J. Breitweg, C. CHekanov, M. Derrick, D. Krakauer, S. Magill, B. Musgrave,

A. Pellegrino, J. Repond, R. Stanek, R. Yoshida

Eur. Physc. J., C18, 625 (2001).

ANL-HEP-PR-01-056

Negative Probability and Uncertainty Relations

T. Curtright and C. Zachos

Mod. Phys. Letts. A 16, 2381-2385, (Dec. 2001).

ANL-HEP-PR-01-034 
Open String Decoupling and Tachyon Condensation

G. Chalmers

Phys. Rev. D.

ANL-HEP-PR-01-012

Pion Interferometry of $\sqrt{s_{N N}}=130 \mathrm{GeV} \mathrm{Au}+\mathrm{Au}$ Collisions at RHIC

T. LeCompte, H. M. Spinka, D. G. Underwood, A. Yokosawa, C. Adler et al., Phys. Rev. Lett. Vol. 87, 082301 (July, 2001).

ANL-HEP-PR-01-032

QCD Factorized Drell-Yan Cross Section at Large Transverse Momentum

E. L. Berger, J.-W. Qiu, and X.-F. Zhang Phys. Rev. D

ANL-HEP-PR-01-057

Resummation of QCD Corrections to the $\eta_{c}$ Decay Rate

G. T. Bodwin and Y.-Q. Chen

Phys. Rev. D

ANL-HEP-PR-01-022

Scaling Behaviour at the $N_{t}=6$ Chiral Phase Transition for 2-Flavour Lattice QCD with Massless Staggered Quarks, and an Irrelevant 4-Fermion Interaction

J. B. Kogut and D. K. Sinclair

Phys. Rev. D

ANL-HEP-PR-01-009

Simplifying Algebra in Feynman Graphs. Part III: Massive Vectors

G. Chalmers and W. Siegel

Nucl. Phys. B

ANL-HEP-PR-00-117

The Muon Anomalous Magnetic Moment - Significance of the New Measurement

J. L. Uretsky

Phys. Rev. Letts.

ANL-HEP-PR-01-049

The New L-Band 1-1/2 Cell Photocathode RF Gun at Argonne

M. E. Conde, W. Gai, R. Konecny, J. G. Power, P. Schoessow, and X. Sun Conference Proceedings. Workshop on Photo-Injector for Energy Recovery Linac, Upton, New York (Brookhaven National Laboratory) Jan.22 - 23, 2001.

ANL-HEP-PR-01-011

The Two Cutoff Phase Space Slicing Method

B. W. Harris and J. F. Owens

Phys. Rev. D

ANL-HEP-PR-00-044 
Two-Colour QCD at Non-Zero Quark-Number Density

J. B. Kogut, D. K. Sinclair, S. J. Hands, and S. E. Morrison

Phys. Rev. D

ANL-HEP-PR-01-028

Upsilon Decay to a Pair of Bottom Squarks

E. L. Berger and L. Clavelli

Phys. Letts.B512, 115-120, (July 2001).

ANL-HEP-PR-01-023

\section{IV.C. PAPERS OR ABSTRACTS SUBMITTED TO CONFERENCES}

A Hybrid Deelectric and Iris Loaded Periodic Accelerating Structure

Peng Zou, Liling Xiao, Xiang Sun, and Wei Gai

Conference Proceedings - Particle Accelerator Conference (PAC2001),

Chicago, IL, June 18-22, 2001.

ANL-HEP-CP-01-064

A Laser Strain Gauge for Accelerator Targets

A.Hassanein, J. Norem

Conference Proceedings - Particle Accelerator Conference (PAC2001), Chicago, IL, June 18-22, 2001, published.

ANL-HEP-CP-01-067

A Supersymmetric Solution to the Bottom-Quark Cross Section Anomaly

Z. Sullivan

XXXVIth Rencontres de Moriond: QCD and High Energy Hadronic

Interactions, Les Arcs, Savoie, France, March 17-24, 2001.

ANL-HEP-CP-01-033

An $\mathrm{e}^{+} \mathrm{e}^{-}$Collider in the VLHC Tunnel

A. Barcikowski, G. Goeppner, J. Norem, E. Rotela, B. Rusthoven, S. Sharma, L. Teng, K. Thompson

Conference Proceedings - Particle Accelerator Conference (PAC2001), Chicago, IL, June 18-22, 2001.

ANL-HEP-CP-01-068

Argonne Wakefield Accelerator Facility Upgrade

M.E. Conde, W. Gai, R. Konecny, J.G. Power, P. Schoessow, X. Sun

Conference Proceedings - Particle Accelerator Conference (PAC2001),

Chicago, IL, June 18-22, 2001.

ANL-HEP-CP-01-058 
Atmospheric Neutrinos in Soudan 2

M. Goodman

Proceedings of the 27th Int'l. Cosmic Ray Conference (ICRC 2001),

Hamburg, Germany, Aug. 7-15, 2001.

ANL-HEP-CP-01-038

Experimental Demonstrations of Two Beam Acceleration Using Dielectric Step-UP Transformer Wei Gai, M.E. Conde, R. Konecny, J.G. Power, P. Schoessow, J. Simpson, X. Sun, and P. Zou

Conference Proceedings - Particle Accelerator Conference (PAC2001), Chicago, IL, June 18-22, 2001, accepted.

ANL-HEP-CP-01-060

5 Accelerator Systems, 5.1 Magnets and vacuum chamber

J. Norem, L. Teng, S. Sharma and O. Grobner

Fermilab Conf. 01/107-T 2001, 67-75, (March 2001)

ANL-HEP-CP-01-088

Field Analysis of a Dielectric-Loaded Rectangular Waveguide Accelerating Structure

Liling Xiao, Wei Gai, and Xiang Sun

Conference Proceedings - Particle Accelerator Conference (PAC2001), Chicago, IL, June 18-22, 2001.

ANL-HEP-CP-01-063

Grid-Enabled Data Access in the ATLAS Athena Framework

D. Malon, E. May, A. Vaniachine

Conference Proceedings

ANL-HEP-CP-01-073

High Power Test of the First S-Band RF Gun at SRRC

W. Gai

Conference Proceedings - Particle Accelerator Conference (PAC2001)

Chicago, IL, June 18-22, 2001.

ANL-HEP-CP-01-059

High-Power Testing of 11,424 GHz Dielectric-Loaded Accelerating Structures W. Gai

Conference Proceedings - Particle Accelerator Conference (PAC2001), Chicago, IL, June 18-22, 2001.

ANL-HEP-CP-01-061

Horizontal Muons in Soudan 2 and Search for AGN ;Neutrinos

D. Demuth (Univ. of Minnesota at Crookstron) and M. Goodman

Proceedings of the $27^{\text {th }}$ Int'l. Cosmic Ray Conference (ICRC 2001),

Hamburg, Germany, Aug. 7-15, 2001.

ANL-HEP-CP-01-039 
Instrumentation for the Muon Based Neutrino Source

J. Norem, C-X Wang

Conference Proceedings - Particle Accelerator Conference (PAC2001),

Chicago, IL, June 18-22, 2001.

ANL-HEP-CP-01-069

Next-to-Leading Order SUSY-QCD Calculation of Associated Production of Gauginos and Gluinos

E. L. Berger, T.M.P. Tait and M. Klasen

$5^{\text {th }}$ International Symposium on Radiative Corrections (RADCOR 2000), Carmel, CA, 11-15 September 2000.

ANL-HEP-CP-01-002

Parameters of an $\mathrm{e}^{+} \mathrm{e}^{-}$Collider in the VLHC Tunnels

J. Norem, S. Sharma, L. Teng

Conference Proceedings, accepted

ANL-HEP-CP-01-096

Physics Goals for the Planned Next Linear Collider Engineering Test Facility

W. Gai, J. Power, and J. Simpson

Conference Proceedings - Particle Accelerator Conference (PAC2001),

Chicago, IL, June 18-22, 2001.

ANL-HEP-CP-01-065

Supersymmetry Contribution to Bottom Quark Production at Hadron Colliders

E. L. Berger

4th International Workshop on B Physics and CP Violation, Ise-Shima, Japan, February 17-23, 2001.

ANL-HEP-CP-01-013

Search for nucleon decay and n-nbar oscillation in Soudan 2

T. Fields, M. Goodman

Proceedings of the 27th Int'l. Cosmic Ray Conference (ICRC 2001),

Hamburg, Germany, Aug. 7-15, 2001.

ANL-HEP-CP-01-037

Thermoelastic Response of Suddenly Heated Liquid Targets in High-Power Colliders

A.Hassanein, I. Konkashbaev, and J. Norem

Conference Proceedings - Particle Accelerator Conference (PAC2001),

Chicago, IL, June 18-22, 2001.

ANL-HEP-CP-01-070

Transformer Ratio Enhancement Using a Ramped Bunch Train in a Collinear Wakefield Accelerator

J.G. Power, W. Gai, and X. Sun

Conference Proceedings - Particle Accelerator Conference (PAC2001),

Chicago, IL, June 18-22, 2001.

ANL-HEP-CP-01-062 
The Radiation Environment in and Near High Gradient RF Cavities

L. Ducas, \& J. Norem

Conference Proceedings - Particle Accelerator Conference (PAC2001),

Chicago, IL, June 18-22, 2001, published.

ANL-HEP-CP-01-066.

TTCPR: APMC Receiver for TTC

John W. Dawson, William N. Haberichter, James L. Schlereth

Conference Proceedings, $7^{\text {th }}$ Workshop on Electronics for LHC Experiments, University of Stockholm, Stockholm, Sweden 10-14, 2001.

ANL-HEP-CP-01-90.

\section{IV.D. TECHNICAL REPORTS AND NOTES}

\section{CDF Notes:}

\section{$\underline{\text { CDF-5238 }}$}

Charge Particle Energy Depositions in the Central Electomagetic Calorimeter S. Kuhlmann, et al.

\section{$\underline{\text { CDF-5342 }}$}

Jet Energy Resolution Studies on a RunIb Di-jet Sample Using Calorimeter, Track and SM Information

S. Kuhlmann, et al.

\section{CDF-5457}

Corrections to the Run 1B Photon Cross Section

S. Kuhlmann, et al.

\section{$\underline{\text { CDF-5518 }}$}

Proposal to Add Timing Information Into the Readout of the Central and Plug Electomagnetic Calorim

R. G. Wagner, et al.

\section{$\underline{\text { CDF-5552 }}$}

Setting the Hadron Calorimeter Energy Scale and the Effect on Jet Resolutions

S. Cullman

\section{$\underline{\text { CDF-5570 }}$}

Search for R-parity Violatuing Decays of Scalar Top Quarks in Two Taus and Two Jets Events

M. Tanaka, et al. 


\section{$\underline{\text { CDF-5636 }}$}

Comparison of the Direct Photon Cross Sections at $\$ \backslash$ sqrt $\{\mathrm{s}\} \$=1.8 \mathrm{TeV}$ and $\$ \backslash$ sqrt $\{\mathrm{s}\} \$=0.63 \mathrm{GeV}$

S. Kuhlmann et al.

\section{CDF-5645}

Search for Radiative B-Hardron Decays with CDR Masashi Tanaka

\section{$\underline{\text { CDF-5657 }}$}

The Central Plug Diphoton Sample

R. Blair, et al.

\section{CDF-5658}

Search for Large Extra Dimensions with the Central-Plug Diphoton Sample R. Blair, et al.

\section{$\underline{\text { CDF-5662 }}$}

Rare and Semileptonic Decays in Run 2

M. Tanaka et al.

\section{CDF-5668}

Inclusive $\mathrm{W}$ and $\mathrm{Z}$ Selection in the $\mathrm{V}+$ Photon Analysis R. G. Wagner et al.

\section{$\underline{\text { CDF-5584 }}$}

A Search for R-parity Violating Scalar Top Quark Decays in Two Tau and Two Jet Events-Muon Channel

M. Tanaka, et al.

\section{NuMI Notes:}

\section{NuMI-L-680}

Sanjib Mishra et al., A description of the THESEUS proposal, Maury Goodman

January 2001 


\section{NuMI-L-757}

Study of QIE Digitization in the Near Detector

David Reyna

June 2001

\section{Wakefield Notes:}

\section{$\underline{\text { WF-202 }}$}

Calculations of Dielectric Loaded Traveling-Wave Periodic Structure Properties Peng Zou, Liling Xiao, Xiang Sun,Wei Gai (ANL) \& Thomas Wong (IIT) $1 / 10 / 01$

\section{$\underline{\text { WF-203 }}$}

The Design of a 13.625 GHz Structure Used for the Transformer Ratio Enhancement Experiments

Xiang Sun \& Wei Gai $1 / 10 / 01$

\section{WF-204}

A NOVEL APPROACH FOR WAKEFIELD MEASUREMENTS AT THE ETF, Jim Simpson 


\section{COLLOQUIA AND CONFERENCE TALKS}

\section{David Ayres}

$16^{\text {th }}$ International School on Particles and Cosmology Baksan, Russia, April 2001

"Soudan 2, 1989-2001, nuclear decay and more."

\section{Edmond L. Berger}

Harry Lipkin, for Pedestrians

Presented at A Celebration of Harry J. Lipkin's Long Association with Argonne National Laboratory, Argonne, IL, June 15, 2001.

Supersymmetry Contribution to Bottom Quark Production at Hadron Colliders Invited talk presented at Frontiers in Contemporary Physics - II, Vanderbilt University, Nashville, TN, March 9, 2001.

Low Energy Supersymmetry and the Bottom-Quark Cross Section Invited plenary talk presented at the 4th International Workshop on B Physics and CP Violation, Ise-Shima, Japan, February 19, 2001.

\section{Gordon Chalmers}

Low Energies Within Maximally Supersymmetric Theories Theory Institute 2001: From Supersymmetry to Extra Dimensions, Argonne, IL, June 19, 2001.

Cosmological Constant and Supersymmetry ANL-HEP Division Seminar, Argonne, IL, May 31, 2001.

Quantum Scattering and Dual Expansions in IIB Superstring Theory Department of Physics, University of Minnesota, Minneapolis, February 2001.

Quantum Scattering and Dual Expansions in IIB Superstring Theory SLAC, Stanford, CA, February 2001.

Quantum Scattering and Dual Expansions in IIB Superstring Theory Department of Physics, University of California, Berkeley, January 2001.

Developments in Supersymmetric Gravity Theories ANL-HEP Division Lunch Seminar, Argonne, IL, January 9, 2001. 


\section{David Kaplan}

Realistic Axions

PHENO 2001 Symposium, Madison, WI, May 8, 2001.

Fermion Masses, Supersymmetry and a Small Extra Dimension

Department of Physics, University of Washington, Seattle, May 1, 2001.

Small Fermion Masses and Spacially Dependent Flat Directions

Fermilab, Batavia, IL, April 5, 2001.

Gaugino-Mediated Supersymmetry Breaking

XXXVth Rencontres de Moriond on QCD and High Energy Hadronic Interactions

Conference, Les Arcs, France, March 2001.

Supersymmetry Breaking and a Small Extra Dimension

Brookhaven National Laboratory, Upton, NY, March 28, 2001.

Fermion Masses, Supersymmetry and a Small Extra Dimension

Department of Physics, Columbia University, New York, NY, March 26, 2001.

Fermion Masses, Supersymmetry and a Small Extra Dimension

Department of Physics, The Johns Hopkins University, Baltimore, MD, March 9, 2001.

Supersymmetry Breaking and a Small Extra Dimension Department of Physics, University of Washington, Seattle, January 2, 2001.

\section{Steve Magill}

$F_{\gamma}^{2}$ Measurements at a Future Linear Collider: $\mathrm{e}^{+} \mathrm{e}^{-}$vs. Gamma-Gamma Invited talk at the Second International Workshop on High Energy Photon Colliders, Fermilab, March 4-17, 2001.

Photon Structure with Bremstrahlung and Backscattered Photons Invited talk at the Workshop on Physics and Detectors for Future $\mathrm{e}^{+} \mathrm{e}^{-}$ Linear Colliders, Johns Hopkins University (March 19-21, 2001).

\section{James Norem}

Neutrino Sources and Muon Collider Argonne, HEP Lunch Seminar, Jan. 23, 2001

Magnets for Large $\mathrm{e}^{+} \mathrm{e}^{-}$Colliders

Workshop on $\mathrm{e}^{+} \mathrm{e}^{-}$Colliders at the VLHC, March 9-11, 2001 
The $\mathrm{e}^{+} \mathrm{e}^{-}$Collider Ring $\mathrm{e}^{+} \mathrm{e}^{-}$Acc Phys \& Tech. Seminar

Fermilab, June 28, 01

\section{Larry Nodulman}

Physics Prospects for CDF in Run II

Centre d'Etudes Nucleaires de Bordeaux-Gradignan

\section{José Repond}

Physics at HERA

Phenomenology 2001 Symposium, Madison, WI, May 7-9, 2001.

\section{Donald K. Sinclair}

Nuclear Matter: A Challenge for Lattice QCD

ANL-HEP Division Seminar, Argonne, IL, March 14, 2001.

\section{Zack Sullivan}

A Supersymmetric Solution to the Bottom-Quark Cross Section Anomaly

Theory Institute 2001: From Supersymmetry to Extra Dimensions, Argonne, IL, June 28, 2001.

Fully Differential NLO QCD Corrections to Single-Top-Quark Production PHENO 2001 Symposium, Madison, WI, May 8, 2001.

Fully Differential NLO QCD Corrections to Single-Top-Quark Production Invited talk presented at the Workshop on Monte Carlo Generator Physics for Run II at the Tevatron, Batavia, IL, April 20, 2001.

A Supersymmetric Solution to the Bottom-Quark Cross Section Anomaly XXXVI Rencontres de Moriond: QCD and Hadronic Interactions, Les Arcs, France, March 21, 2001.

Direct Probes of R-Parity Violation at the Tevatron

Department of Physics, Southern Methodist University, TX, February 19, 2001. 


\section{Timothy Tait}

Fermion Masses, Supersymmetry and a Small Extra Dimension

Theory Institute 2001: From Supersymmetry to Extra Dimensions, Argonne, IL, June 27, 2001.

Supersymmetry and the Bottom Quark Cross Section

Joint Theoretical/Experimental Colloquium, FNAL, Batavia, IL, June 1, 2001.

Could We Already be Seeing Supersymmetry at Hadron Colliders?

PHENO 2001 Symposium, Madison, WI, May 8, 2001.

Extra-Dimensional EWSB and Fermion Masses

Parallel Session, Workshop on the Future of Higgs Physics, Batavia, IL, May 4, 2001.

Flavor and SUSY Breaking From a Small Extra Dimension

Department of Physics, Michigan State University, East Lansing, MI, February 13, 2001.

Why the World Doesn't Fall Apart: The Mystery of Electroweak Symmetry Breaking

Tevatron University Lecture, FNAL, Batavia, IL, January 18, 2001.

\section{Carlos Wagner}

Brane Effects on Extra Dimensional Scenarios

Theory Institute 2001: From Supersymmetry to Extra Dimensions, Argonne, IL, June 2001.

The Anomalous Magnetic Moment of the Muon as a Probe of the Standard Model of Particle Interactions

Physics Division Colloquium, Argonne, IL, June 8, 2001.

Introduction to Supersymmetry

Department of Physics, University of Chicago, Illinois, May 2001.

New Physics at the Tevatron

PHENO 2001 Symposium, Madison, WI, May 8, 2001.

Higgs Physics and the Origin of Baryon Asymmetry in the MSSM

Parallel Session, Workshop on the Future of Higgs Physics, Batavia, IL, May 5, 2001.

Higgs Physics and the Origin of Baryon Asymmetry in the MSSM

DESY, Hamburg, Germany, April 2001.

Electroweak Baryogenesis in the MSSM

Workshop on Cosmology in Supersymmetry and String Theory, Heidelberg, Germany, April 2001. 
Electroweak Baryogenesis in the MSSM

Higgs and Supersymmetry, Orsay, France, March 2001.

Supersymmetry at Photon Colliders

Workshop on Physics at Photon Colliders, Fermilab, Batavia, March 2001.

On the Anomalous Magnetic Moment of the Muon

Department of Physics, University of Chicago, Illinois, March 2001.

Higgs Physics and CP Violation

Department of Physics, University of Chicago, Illinois, February 2001.

A Tale of Two Gravitons

Workshop on Physics in Extra Dimensions, Warsaw, Poland, February 2001.

\section{Rik Yoshida}

QCD at Colliders

Invited talk at the German Physical Society Meeting, Bonn Germany, March 27, 2001.

\section{Arthur B. Wicklund}

"B Physics at the Tevatron" (invited talk)

Aspen winter conference on particle Physics, Aspen, Jan. 2001. 


\section{HIGH ENERGY PHYSICS COMMUNITY ACTIVITIES}

\section{David Ayres}

Deputy Spokesperson, MINOS Collaboration Organizing Committee "Workshop on the Brighter Booster" September 2001, Fermilab.

Encoder for Particle Data Group

\section{Edmond L. Berger}

Consultant, Department of Energy Review of the High Energy Physics Program at the Stanford Linear Accelerator Center, April 3-5, 2001.

Organizing Committee, Theory Institute on SUSY and Extra-Dimensions Argonne National Laboratory, June 17-29, 2001.

Member, High Energy and Nuclear Physics Advisory Committee Brookhaven National Laboratory, 1995-2001.

Member, CTEQ Collaboration

Adjunct Professor of Physics, Michigan State University East Lansing, MI, 1997-present.

Co-host with Steve Kuhlmann of CTEQ collaboration meeting at Argonne October 26-27, 2001.

Scientific Program Organizing Committee, XXXVI Rencontres de Moriond, QCD and High Energy Hadronic Interactions, France, March, 2001.

Scientific Advisory Committee, Frontiers in Contemporary Physics-II Vanderbilt University, March 5-10, 2001.

International Advisory Committee, 9th International Conference on Hadron Spectroscopy (HADRON2001), Protvino, Russia, August 25--September 1, 2001.

Scientific Program Organizing Committee, XXXVIIth Rencontres de Moriond, QCD and High Energy Hadronic Interactions, France, March, 2002.

Convenor for QCD, Division of Particles and Fields Meeting, DPF 2002 Williamsburg, May, 2002. 
International Advisory Committee, Conference in Honor of Jean Tran Thanh Van Paris, October, 2002.

Organizing Committee, 8th Conference on the Intersections of Particle and Nuclear Physics (CIPANP 2003), New York, May, 2003.

\section{Gordon Chalmers}

Organizing Committee, Theory Institute on SUSY and Extra-Dimensions Argonne National Laboratory, June 17-29, 2001.

\section{Wei Gai}

Member, Scientific and Organizing Committee, Advanced Acceleration Concepts Workshop 2001.

\section{Brian Harris}

Organizing Committee, Theory Institute on SUSY and Extra-Dimensions Argonne National Laboratory, June 17-29, 2001.

\section{David Kaplan}

Organizing Committee, Theory Institute on SUSY and Extra-Dimension Argonne National Laboratory, June 17-29, 2001.

\section{Larry Nodulman}

Chair of the Fermilab Users Executive Committee

\section{Jim Norem}

Organized Workshop on an e+e- ring at the VLHC

Illinois Institute of Technology, Chicago IL, March 10, 2001

\section{José Repond}

Member, International Advisory Committee for the $10^{\text {th }}$ International Workshop on Deep Inelastic Scattering and QCD, Cracow, Poland, September 14-18, 2001 


\section{Zack Sullivan}

Organizing Committee, Theory Institute on SUSY and Extra-Dimensions Argonne National Laboratory, June 17-29, 2001.

\section{Timothy Tait}

Organizing Committee, Theory Institute on SUSY and Extra-Dimensions Argonne National Laboratory, June 17-29, 2001.

\section{Carlos Wagner}

Organizing Committee, Theory Institute on SUSY and Extra-Dimensions Argonne National Laboratory, June 17-29, 2001.

\section{Cosmas Zachos}

Member, Advisory Panel for J. Phys. A (Math. Gen). 


\section{HEP DIVISION RESEARCH PERSONNEL}

\section{Administration}

Price, L.

Accelerator Physicists

Conde, $\mathrm{M}$.

Gai, W.

Norem, J.
Hill, D.

Power, J.

Schoessow, P.

\section{Experimental Physicists}

Ayres, D.

Blair, R.

Byrum, K.

Chekanov, S.

Derrick, M.

Fields, T.

Goodman, M.

Joffe-Minor, T.

Krakauer, D.

Kuhlmann, S.

LeCompte, $\mathrm{T}$.

Magill, S.

May, E.

Musgrave, B.

Nodulman, L.
Pellegrino, A.

Proudfoot, J.

Repond, J.

Reyna, D.

Spinka, H.

Stanek, R.

Talaga, R.

Tanaka, M.

Thron, J.

Underwood, D.

Wagner, R.

Wicklund, A.

Yokosawa, A.

Yoshida, R.

\section{Theoretical Physicists}

Berger, E.

Bodwin, G.

Chalmers, G.

Harris, B.

Kaplan, D.

Sinclair, D.
Sullivan, Z.

Tait, $\mathrm{T}$.

Wagner, C.

White, A.

Zachos, C.

\section{Engineers and Computer Scientists}

Dawson, J.

Drake, G.

Grudzinski, J.

Guarino, V.
Hill, N.

Kovacs, E.

Mouser, C.

Schlereth, J.

Vaniachine, A. 
Adams, C.

Ambats, I.

Anderson, S.

Caird, A.

Cox, G.

Cundiff, T.

Franchini, F.

Haberichter, W.

Jankowski, D.

Kasprzyk, T.
Kocenko, L.

Konecny, R.

Matijas, Z.

Nephew, T.

Reed, L.

Rezmer, R.

Rice, J.

Skrzecz, F.

Wood, K.

\section{Laboratory Graduate Participants}

Bell, W.

Zou, P.
Breitweg, J.

\section{Visiting Scientists}

Choudhury, D. (Theory)

Lipkin, H. (Theory)

Crittenden, J. (ZEUS)

Ramsey, G. (Theory)

Hakioglu, T. (Theory)

Sun, X. (AWA)

Kovacs, E. (Theory)

Uretsky, J. (Theory)

Krueger, K. (STAR)

Xiao, L. (AWA) 